\title{
PROMPT NEUTRINO PRODUCTION IN 400 GeV PROTON-COPPER INTERACTIONS
}

\author{
The CHARM Collaboration
}

J. Dorenbosch

NIKHEF, Amsterdam, The Netherlands

\author{
J.V. Allaby, U. Amaldi, G. Barbiellini' ${ }^{1)}$, F. Bergsma, A. Capone ${ }^{2)}$, W. Flegel, L. Lanceri ${ }^{3)}$, \\ C. Nieuwenhuis, J. Panman, C. Santoni ${ }^{4)}$ and K. Winter \\ CERN, Geneva, Switzerland \\ I. $\mathrm{Abt}^{5)}$, J. Aspiazu, F.W. Büsser, H. Daumann, P.D. Gall, T. Hebbeker"), \\ F. Niebergall, P. Schütt and P. Stähelin \\ II. Institut für Experimentalphysik ${ }^{7)}$, Universität Hamburg, Hamburg, Germany \\ P. Gorbunov, E. Grigoriev, V. Khovansky, S. Krutchinin, A. Maslennikov and A. Rosanov \\ Institute for Theoretical and Experimental Physics, Moscow, USSR \\ L. Barone ${ }^{8)}$, B. Borgia ${ }^{8)}$, M. Diemoz ${ }^{8)}$, U. Dore ${ }^{8)}$, F. Ferroni ${ }^{8)}$, E. Longo ${ }^{8)}$, L. Luminari ${ }^{8)}$, \\ P. Monacelli ${ }^{8)}$, F. de Notaristefani ${ }^{8)}$, R. Santacesaria ${ }^{8)}$ and V. Valente ${ }^{\text {9) }}$ \\ Istituto Nazionale di Fisica Nucleare, Rome, Italy
}

(Submitted to Zeitschrift für Physik)

1) Now at the Department of Physics, University of Trieste, Italy.

2) On leave of absence from Università 'La Sapienza', Rome, Italy.

3) Now at INFN, Trieste, and the University of Udine, Italy.

4) Now at the University of Basle, Switzerland.

5) Now at SLAC, Stanford, Calif., USA.

6) Now at CERN, Geneva, Switzerland.

7) Funded by the German Federal Ministry for Research and Technology (BMFT) under contract number $4 \mathrm{HH} 22 \mathrm{P}$.

8) Dipartimento di Fisica, Università 'La Sapienza', Rome, and INFN Sezione di Roma, Rome, Italy.

9) Laboratori Nazionali INFN, Frascati, Italy. 


\begin{abstract}
A new beam-dump experiment has been performed at the CERN Super Proton Synchrotron using the CHARM neutrino detector. The instrumentation and the statistics have been significantly improved with respect to earlier experiments. For a neutrino energy above $20 \mathrm{GeV}$ the asymmetry of the prompt muon-neutrino and electron-neutrino fluxes $\left[\left(\nu_{\mu}+\bar{\nu}_{\mu}\right)-\left(\nu_{\mathrm{e}}+\bar{\nu}_{\mathrm{e}}\right)\right] /\left[\left(\nu_{\mu}+\bar{\nu}_{\mu}\right)+\right.$ $\left.\left(\nu_{\mathrm{e}}+\bar{\nu}_{\mathrm{e}}\right)\right]$ is found to be $0.20 \pm 0.10$ (stat.) \pm 0.05 (syst.), and the asymmetry of prompt antineutrino and neutrino fluxes for muon-neutrinos $\left(\nu_{\mu}-\bar{\nu}_{\mu}\right) /\left(\nu_{\mu}+\bar{\nu}_{\mu}\right)$ is $0.02 \pm 0.16$ (stat.) \pm 0.02 (syst.), in agreement with our previous results. For the cross-section times branching ratio for charm production and semileptonic decay we obtain a value of $\sigma \times \operatorname{BR}\left[\mathrm{D}(\overline{\mathrm{D}}) \rightarrow \nu_{\mathrm{e}}\left(\bar{\nu}_{\mathrm{e}}\right) \mathrm{X}\right]=1.9 \pm$ $0.2 \pm 0.2 \mu \mathrm{b}$ per nucleon. We find no evidence for $\nu_{\tau}$ or $\nu_{\mathrm{X}}$ interactions. The $\left(\nu_{\tau}+\bar{\nu}_{\tau}\right)$ flux is less than $21 \%$ of the total prompt neutrino flux. We derive an improved limit on the branching ratio $\pi^{0} \rightarrow \nu \bar{\nu}$ of $6.5 \times 10^{-6}$, and as a verification of the universality of the neutral weak coupling we find $\mathrm{g}_{\nu_{\mathrm{e}} \bar{\nu}_{\mathrm{e}}} / \mathrm{g}_{\nu_{\mu} \bar{\nu}_{\mu}}=1.05 \pm 0.15$.
\end{abstract}




\section{INTRODUCTION}

Beam-dump experiments were first proposed by Bruno Pontecorvo to search for short-lived particles. Today this technique is still very adequate for making truly inclusive measurements, for instance of the ratio of electron-neutrino $\left(\nu_{\mathrm{e}}\right)$ to muon-neutrino $\left(\nu_{\mu}\right)$ decays of short-lived charmed hadrons.

In such an experiment the beam impinges on a solid block of dense material. The particles that are created undergo secondary interactions before they decay, if their lifetime is longer than the time needed to cover the distance of an interaction length. The flux of decay products from these particles can thus be reduced by several orders of magnitude. This eases the detection of decay products of short-lived particles, in particular if these particles are produced with a low cross-section.

In a beam-dump experiment carried out at the CERN Super Proton Synchrotron (SPS) in 1979, earlier evidence for the production of prompt $\nu_{\mathrm{e}}$ was confirmed and the existence of a flux of prompt $\nu_{\mu}$ and $\bar{\nu}_{\mu}$, in roughly equal proportions, was established [1-3]. Here we report results from a beam-dump experiment performed in 1982 at the CERN SPS, mainly in order to investigate further whether the ratio of prompt $\nu_{\mathrm{e}}$ to $\nu_{\mu}$ fluxes is smaller than unity as reported by us [2] and by two other groups, the Aachen-Bonn-CERN-London-Oxford-Saclay (ABCLOS) Collaboration [1] and the CERN-Dortmund-Heidelberg-Saclay (CDHS) Collaboration [3]. Preliminary results from the new experiment have already been reported [4].

Similar experiments have been performed by Duffy et al. at Fermilab [5]. Results from the BEBC Collaboration on an experiment using the same beam and target arrangement as described in this paper are published in ref. [6], and preliminary results of the CERN-Dortmund-HeidelbergSaclay-Warsaw (CDHSW) Collaboration in ref. [7].

Our experiment was performed using a new beam installation. The target station was placed closer to the detector, thus increasing the solid angle of acceptance. The beam monitoring was extended, especially the measurement of proton losses upstream of the target. For normal operation the beam was guided through vacuum, from the accelerator up to immediately before the target, with no material (such as windows or monitors) in the beam line. Finally, the statistical precision obtained for the basic rates is about six times higher than in the previous experiment.

The aim of the experiment is to determine the flux of neutrinos from sources with a short lifetime $\left(\leq 10^{-11} \mathrm{~s}\right)$ and to search for other kinds of penetrating particles. The neutrinos from these short-lived parents are referred to as 'prompt'. Contributions of neutrinos from sources with a longer lifetime $(\pi, \mathrm{K}$, etc.) are strongly suppressed because of the high density of the dump target. The flux component due to these sources is called 'conventional'. To distinguish between these components, we applied two different methods.

In the first method, the data obtained with two targets of different average density were used to extrapolate to infinite density in order to obtain the prompt component (extrapolation method). For this method the relative target densities have to be well known, and the experimental conditions for running at different densities have to be the same. A precise knowledge of the relative beam intensity normalization is essential.

In the second method the conventional fluxes were determined from measurements of various components of the muon flux in the iron shield behind the target, combined with Monte Carlo calculations. This flux was then subtracted from the data to yield the prompt rates (subtraction method). The method relies on an accurate measurement of the muon fluxes in the iron shield and on a reliable simulation of the hadron cascade of the parents of the neutrinos and the muons.

The experiment measures the flux of prompt $\nu_{\mathrm{e}}$ and $\nu_{\mu}$ by detecting their charged-current (CC) interactions. The identification of a muon among the neutrino interaction products can be taken (up to small corrections) as an indicator of a CC $\nu_{\mu}$ or $\overline{\nu_{\mu}}$ interaction. The determination of its charge allows to distinguish $\nu_{\mu}$ from $\bar{\nu}_{\mu}$ events. Charged-current $\nu_{\mathrm{e}}$ interactions can be selected from the 
sample of events without a muon, either by detecting an electromagnetic shower directly or by making use of the known ratio of the neutral current to charged current cross-section, assuming this ratio to be equal for $\nu_{\mathrm{e}}$ and $\nu_{\mu}$.

The CHARM neutrino detector is well suited to the identification of $\mathrm{CC} \nu_{\mu}$ interations down to very low muon momenta. However, for this experiment it is particularly important to be able to identify also $\mathrm{CC} \nu_{\mathrm{e}}$ interactions. Since the rate of neutral current (NC) interactions can be well predicted from the measured $\mathrm{CC}$ rate, we hereby have the possibility to search for interactions of penetrating particles other than the $\nu_{\mu}$ and the $\nu_{\mathrm{e}}$. The alternative method of determining the rate of $\nu_{\mathrm{e}}$ interactions, by simply assuming that all events not accounted for by $\nu_{\mu}$ are due to $\nu_{\mathrm{e}}$, is also applied and used as a cross-check.

The muon flux originating in the beam-dump target was measured in the downstream iron shield. These measurements were analysed to cross-check the results of prompt neutrino interactions and, combined with the neutrino results, to set a limit on the production of short-lived particles decaying into $\mu+\nu+\mathrm{X}$ and on the branching ratio of $\pi^{0}$ decaying into a $\nu \bar{\nu}$ pair.

The normal neutrino detector was supplemented by a special low-mass set-up which was used to search for the decay of axions and of heavy neutrinos. The results of these experiments have already been published [8].

The experimental set-up is described in section 2 . We discuss data-taking, analysis, and corrections in sections 3 and 4 , and the determination of the conventional neutrino fluxes in section 5. Results are presented in section 6 and discussed in section 7 .

\section{THE EXPERIMENTAL SET-UP}

\subsection{The beam and the targets}

As in the previous experiment [2], a $400 \mathrm{GeV}$ proton beam was extracted from the CERN SPS in a single turn $(23 \mu \mathrm{s})$ and, pointing towards the neutrino detector, was dumped into a thick target. Targets were installed at the downstream end of the wide vacuum pipe which serves as decay tunnel for normal neutrino beam operation. The distance to the centre of the fiducial volume of the detector was $487.3 \mathrm{~m}$ (fig. 1).

Figure 2 shows details of the target arrangement. For normal data-taking we used copper targets with average relative densities of $\varrho=1$ (solid copper) and $\varrho=1 / 3$ and, in addition, of $\varrho=1 / 2$ for some special runs for muon flux measurements. The targets have a cross-section of $41.5 \mathrm{~cm} \times 31 \mathrm{~cm}$ and are $302.5 \mathrm{~cm}$ long. The lower densities of the targets $(\varrho=1 / 2$ and $1 / 3)$ were obtained by spacing $2.5 \mathrm{~cm}$ thick pieces of solid copper with air gaps of $2.5 \mathrm{~cm}$ or $5 \mathrm{~cm}$ over the full target length $(\varrho=1 / 3)$ or over two thirds $(\varrho=1 / 2)$ of the total length, respectively. This structure was obtained by milling slits into a copper block. These targets were followed by a $302.5 \mathrm{~cm}$ long solid copper block - the hadron catcher - with lateral dimensions of $41.5 \mathrm{~cm} \times 41.5 \mathrm{~cm}$. There is a $25 \mathrm{~mm}$ air gap between the target and the catcher.

The proton beam intensity incident on the target was measured by three beam current transformers (BCTs), placed $300 \mathrm{~m}, 520 \mathrm{~m}$, and $550 \mathrm{~m}$ upstream of the target. The beam position was continuously monitored by secondary-emission-grid chambers located immediately in front of the dumps. The alignment of the ejected proton beam pointing towards the neutrino detector was checked by measuring the muon flux, originating in the target, in rings of solid-state detectors inserted into narrow gaps of the iron shield. These counters were also used to monitor the muon flux (see section 3 ).

The largest potential source of background in the determination of the prompt $\nu_{\mu}$ flux is due to scraping of the proton beam. Even a small fraction of protons interacting upstream of the dump would increase the conventional flux in a dangerous way owing to the presence of a long free path for the decay of the secondaries. Moreover, since this background does not depend on the density of the 
target, it cannot be eliminated directly by extrapolation and would be interpreted as prompt. Twenty-six ionization chambers were installed pairwise along the beam line, left and right and above and below the vacuum chamber, in order to adjust the beam optics so as to avoid scraping and to monitor the flux of secondaries which could be produced by scraping. The currents in these chambers were recorded continuously during data-taking. The scraping monitors were calibrated by flipping aluminium foils of $7.1 \mu \mathrm{m}, 14.1 \mu \mathrm{m}$, and $80.6 \mu \mathrm{m}$ thickness into the proton beam at about $440 \mathrm{~m}$ upstream of the target, thereby inducing inelastic interactions with probabilities of $1.8 \times 10^{-5}$, $3.6 \times 10^{-5}$ and $20.5 \times 10^{-5}$ per proton, respectively. Relating the results of this calibration to the observed rate of neutrino interactions from the normal neutrino beam operation on a target at the same location, we estimated that inelastic proton interactions in a $10 \mu \mathrm{m}$ Al foil produce a neutrino flux in the detector corresponding to $1 \%$ of the expected [2] prompt flux.

\subsection{The detector}

The CHARM neutrino detector has been described before $[9,10]$. It is composed of a fine-grain target calorimeter and of a muon spectrometer. The target calorimeter consists of 72 marble plates of $8 \mathrm{~cm}$ thickness and $3 \times 3 \mathrm{~m}^{2}$ cross-section which are interleaved between layers of scintillation counters $\left(3 \mathrm{~cm}\right.$ thick, $15 \mathrm{~cm}$ wide, and $300 \mathrm{~cm}$ long) and proportional drift tubes $\left(3 \times 3 \times 400 \mathrm{~cm}^{3}\right)$. The proportional drift tubes extend into the $45 \mathrm{~cm}$ wide magnetized iron frame that surrounds the marble plates. In front of each of the marble target plates a plane of digital wire chambers working in the limited streamer mode [11], with a wire distance of $1 \mathrm{~cm}$, covered a surface of $285 \times 265 \mathrm{~cm}^{2}$, the wires being oriented at $90^{\circ}$ with respect to those of the proportional drift tubes. The orientation of all detector elements alternates between horizontal and vertical in consecutive gaps.

The main features of this detector are its high efficiency for detecting showers even at low energies $\left(\epsilon>99 \%\right.$ for $\left.E_{s h} 1.5 \mathrm{GeV}\right)$, its good hadron energy resolution $\left[\sigma\left(E_{h}\right) / E=0.49 / \sqrt{E}_{h}\left(E_{h}\right.\right.$ in $\mathrm{GeV})$ ], and its pattern recognition capabilities allowing, for example, the detection of muons with momenta as low as $1 \mathrm{GeV} / \mathrm{c}$ in $\mathrm{CC} \nu_{\mu}$ events. Owing to this feature, events can be classified as $\mathrm{CC}$ or $\mathrm{NC}$ interactions by direct recognition of the muon on an event-by-event basis. Charged-current events initiated by $\nu_{\mathrm{e}}$ can be recognized because of the characteristic difference in the lateral profile of electron- and hadron-induced showers.

The capabilities of the detector thus allow the classification of events into three categories:

i) $\mathrm{CC} \nu_{\mu}$ and $\bar{\nu}_{\mu}$ events $[\mathrm{N}(1 \mu)]$;

ii) $\mathrm{CC} \nu_{\mathrm{e}}$ and $\bar{\nu}_{\mathrm{e}}$ events [N(1e)]; and

iii). muonless events $[\mathrm{N}(0 \mu)]$, which include the (1e) events.

Using these categories we can determine the fluxes of prompt $\nu_{\mu}$ and $\bar{\nu}_{\mu}$ and prompt $\nu_{\mathrm{e}}+\bar{\nu}_{\mathrm{e}}$, and of any excess not accounted for by the interaction of these known neutrinos.

The part of the calorimeter used as the fiducial volume for the vertex of neutrino interactions had a cross-section of $2.4 \times 2.4 \mathrm{~m}^{2}$ and extended from target plane 3 to target plane 59 , out of the total of 72 planes. The total mass of the fiducial volume is $90.6 \mathrm{t}$. The other parts of the calorimeter volume have only detector functions. The first two planes are used to veto incident muons; the lateral iron frames and the outer $30 \mathrm{~cm}$ of the calorimeter planes veto cosmic-ray muons and are also used for shower measurements. The last 14 planes (corresponding to 14 radiation lengths) and the end calorimeter are used for shower measurements and event classification. The response and resolution of the calorimeter to hadronic and electromagnetic showers has recently been measured in the energy range from 1 to $140 \mathrm{GeV}[10]$.

Table 1 summarizes the main parameters of the experimental set-up. Figure 3 shows schematic views of a $\mathrm{CC}$ and a $\mathrm{NC}$ neutrino event. Scintillation counters and proportional drift tubes which are hit by particles of the event are shown as a line and as a dot respectively. The range of a $1 \mathrm{GeV}$ muon is indicated. Streamer-tube hits are not displayed in this figure. 


\section{DATA-TAKING}

The neutrino detector was triggered by hits of at least four scintillator planes. This condition ensured full efficiency for showers with energies above $1.5 \mathrm{GeV}$ and for almost all events which contain a muon of more than $200 \mathrm{MeV}$ energy irrespective of the shower energy. Events were recorded during the $23 \mu \mathrm{s}$ spill-time of the SPS. Those recorded outside that time were used to measure the cosmic-ray background.

The polarity of the magnetic field, both of the frame magnet and of the end magnet system, was changed regularly every few hours in order to record an equal amount of data under focusing conditions that were favourable to $\mu^{+}$and to $\mu^{-}$.

Data were accumulated with protons on the targets with $\varrho=1$ and $1 / 3$ in the proportion of about two to one in order to optimize the statistical accuracy of prompt events (see table 1).

Up to three events could be recorded per accelerator cycle. We monitored the resulting effective dead-time of the detector by using the signals from two different scintillation counters which we installed in the gaps in the iron shield. These counters detected single muons originating in the dump target. The dead-time was recorded for each burst and amounted to an average of $13.6 \%$ for the $\varrho=1$ and $21.7 \%$ for the $\varrho=1 / 3$ target.

Beam information was collected by a dedicated on-line computer, sent via a data link to the experiment, and recorded, together with the data from the detector, for each individual burst. The most important information concerning the beam was the intensity of protons on the target as measured with three different BCTs, the beam position, measured by secondary-emission-grid detectors immediately in front of the dump target, and the information on beam scraping as recorded by the set of 26 beam loss monitors around the beam pipe. The intensity of the muon flux in the iron shield, as measured by the solid-state detectors [12], was also recorded for each burst. The vacuum in the decay tunnel was continuously monitored, and signals were transmitted which indicated whether equipment was in the beam line and if the correct target was in the nominal position. All monitors were calibrated at regular intervals.

We performed special runs to measure the muon flux and its radial dependence using solid-state detectors at five different depths in the iron shield, corresponding to $2.9 \mathrm{~kg} / \mathrm{cm}^{2}, 17.7 \mathrm{~kg} / \mathrm{cm}^{2}$, $32.2 \mathrm{~kg} / \mathrm{cm}^{2}, 47.0 \mathrm{~kg} / \mathrm{cm}^{2}$, and $64.4 \mathrm{~kg} / \mathrm{cm}^{2}$ of iron. These measurements were performed for the three targets of different density to determine (by extrapolation to infinite density) the muon fluxes of conventional and prompt origin. Prompt muons are mainly due to electromagnetic decays of mesons and to semileptonic decays of charmed particles.

The absolute calibration of the solid-state detectors in pits 2,4 , and 5 was achieved by measurements with a scintillation counter telescope incorporating tungsten absorbers to eliminate the soft electron component. These calibration data were taken in special runs of low intensity and increased spill length. In some runs the catcher behind the dump (see fig. 2) was removed in order to determine the effective average density of the $\varrho=1 / 3$ target, which was slightly more than $1 / 3$ because of the limited length of the laminated section ${ }^{*}$.

Figure 4 shows the results of these measurements. The three solid curves represent the muon fluxes for the three different targets, integrated over the whole solid angle and normalized to $10^{10}$ protons on the target. The curves are calculated interpolations between the data points, indicated by full dots for measurements with the catcher in position, and by crosses for those with the catcher removed. Catcher-out points are also used to determine additional points on the curves for the $\varrho=1$ and $\varrho=1 / 2$ targets. For the $\varrho=1 / 3$ target the catcher-out data are visibly above the curve, indicating a leakage of hadrons out of the target and their decay in the free space after the target. The deviation of these data points from the curve was used to calculate the muon flux due to leakage

*) We are grateful to $\mathrm{W}$. Venus and $\mathrm{H}$. Wachsmuth for providing us with the data necessary for this analysis. 
from the $\varrho=1 / 3$ target. The resulting curves for the leakage flux and for conventional and prompt muon fluxes obtained by extrapolation to infinite density are also shown in fig. 4.

The consistency of this method of muon flux determination is illustrated in fig. 5. It shows the extrapolation to infinite density of the muon fluxes measured in different pits. The flux measured for $\varrho=1 / 3$ is leakage-corrected and normalized to one for all pits, and is extrapolated to infinite density using the data point for $\varrho=1$. The data points for $\varrho=1 / 2$ are seen to be consistent with the linear extrapolation.

The flux of conventional neutrinos from the leakage of hadrons from the $\varrho=1 / 3$ target was calculated by a Monte Carlo simulation which uses the muon leakage flux as input. The corrections to be applied for extrapolating the $e=1$ neutrino data are summarized in table 2 . They are given as the fraction of the conventional neutrino event rate for the $\varrho=1$ target which appears to be prompt. As an example: $(5.5 \pm 0.5) \%$ of the conventional neutrino event rate with $\mathrm{E}_{\nu} \geq 2 \mathrm{GeV}$ for $\varrho=1$ appears to be prompt because of leakage. Above $\mathrm{E}_{\nu}=80 \mathrm{GeV}$ the leakage effect is reduced to $(2.8 \pm 0.3) \%$.

Special tests have been performed to ensure that the effective density of the copper targets was not affected by heating up, shock waves, or long-term damage of the target structure. The time constant for heating up the target is about 1.3 hours. We compared the muon rates measured with the hot and the cold target and found that the heating effect on the effective density is less than $0.1 \%$. The comparison of muon rates for low and high proton intensities shows that shock waves caused by the protons hitting the target change the density by less than $0.1 \%$. The monitoring of muon rates over the whole running period shows random fluctuations of $\pm 0.5 \%$, indicating that a possible change of the effective target density with time must be smaller than this value. Monte Carlo calculations showed that the finite granularity of the $e=1 / 3$ target causes a deviation from the nominal $Q=1 / 3$ density by less than $0.1 \%$. To account for all these uncertainties and for the tolerances of the mechanical construction of the $\varrho=1 / 3$ target, we assumed a total systematic error of $\pm 0.5 \%$ for the density. This error was taken into account for all rates obtained by extrapolation.

\section{DATA ANALYSIS}

\subsection{Beam monitoring and dead-time determination}

The information from the beam monitors was processed in parallel with the events on a burst-by-burst basis. Data were eliminated from further analysis if the monitor information was not available (for example, because of malfunctioning of the data link), if any kind of equipment was in the beam line (for example, for special beam steering or monitor calibration), or if the measured beam losses were above the threshold of a possible contribution of $1 \%$ of the expected prompt rate. In addition, some runs were eliminated because of technical problems with the detector or with the beam operation. Table 3 shows the break-up of the data into these various categories.

A typical example of a distribution of proton loss rates, as measured for individual bursts in one of the loss monitors, and of the cut applied is shown in fig. 6a. The total proton intensity for different targets is established by integration of the BCT rates over all bursts retained for analysis. The stability of the BCT chosen as the main monitor was checked over the whole period of running by comparing its reading with those of other monitors. Figures $6 \mathrm{~b}$ and $\mathrm{c}$ show this comparison with another BCT and with a solid-state detector, respectively, for roughly half of the total running time. The comparison of the BCT with a solid-state detector in the muon shield shows two different peaks because the muon flux changes with target density. As a result of these tests we conclude that fluctuations of the proton intensity measurements are less than $\pm 0.5 \%$.

The live-time of the detector was typically $86.4 \%$ for the $\varrho=1$ target and $78.3 \%$ for the $\varrho=1 / 3$ target. Dead-time effects introduce an important correction of the normalization of events to protons on target (POT); we therefore applied several methods in order to determine the dead-time. The most 
reliable method uses signals from small scintillation counters installed in the iron shield downstream from the dump target. These counters detect muons from decaying secondaries of the proton interactions in the target; hence the signal frequency reflects the time structure of the proton intensity. It was checked that the signal rate is proportional to the proton intensity to a precision of better than $0.5 \%$. Intensity variations during the beam spill are therefore taken into account by this method. The fractional live-time is calculated as the ratio of counts recorded during the beam gate of $33 \mu \mathrm{s}$ when the detector is not busy, to the total number of counts recorded in that gate. Another measurement using a constant-frequency clock signal was always performed in parallel to monitor the stability of the method.

In addition, we calculated the live-time of the detector from the distribution of triggers per burst, independently of additional signals and timings. The proton spill was $23 \mu \mathrm{s}$ long, and we used a time gate of $33 \mu \mathrm{s}$. As the dead-time of the detector is $15 \mu \mathrm{s}$ per trigger, we can record a maximum of three events per burst. The distribution of the number of events per burst is narrower than the corresponding Poisson distribution owing to the dead-time effect [13].

We used Monte Carlo methods to simulate the live-time measurement and to check and monitor the experimental value. Input to the program were: the instrumental values of the dead-time $-15 \mu \mathrm{s}$ per trigger and 200 ns per strobe and veto hit; the average number of triggers and veto hits per incident proton; and the average time-dependent proton intensity of the spill as measured by recording the arrival time of triggers during the gate. The dead-time was simulated for each individual burst, using the proton intensity measured by the BCT.

Table 4 shows a comparison of various typical dead-time values for one individual run. As can be seen from this table, the scintillator and the Poisson method are in good agreement, and the Monte Carlo simulation reproduces well all three experimental values. The slightly lower dead-time obtained by using the clock signal is due to neglect of the intensity variation over the spill-time.

From the differences in the dead-time evaluations we estimate that the error on the live-time measurement translates to a $\pm 0.8 \%$ random uncertainty of the corrected number of POT.

For correct event normalization it is also important that the gate used for event recording covers the beam spill completely. We monitored the gate position by recording the signals from the scintillation counters in the shield in the $33 \mu$ s time gate for data-taking, as well as in a wider gate $(10 \mathrm{~ms})$ extending symmetrically from the short gate. The difference in counting rate, integrated over the whole experiment, is less than $0.1 \%$ and is compatible with noise counts in the scintillators.

The time dependence of the event rate per incident proton over the duration of the whole experiment, which lasted, with interruptions, over a period of more than five months, is shown in fig. 7. We eliminated bursts that could not be used for analysis. The statistical errors are increased by a systematic error of $\pm 0.5 \%$ for normalization to POT and $\pm 0.8 \%$ due to uncertainties in the live-time measurement. A statistical test of these data [14] showed perfect agreement with the hypothesis of random fluctuations of the individual rates about the mean value.

Another way of checking the consistency of the measured rates is shown in fig. 8: the distribution of the deviation of event rates for individual runs from the mean rate normalized to the individual errors is given. The histogram can be well described by a Gaussian distribution centred at $-0.01 \pm 0.08$ with a width of $1.09 \pm 0.06$, in excellent agreement with the expected centre position of 0 and a width of 1 . This agreement also indicates that the error estimates at this level of analysis are realistic.

Taking the different uncertainties into account, we obtained the dead-time-corrected number of POT for the $\varrho=1$ and $\varrho=1 / 3$ targets given in table 1 . The errors indicate the relative uncertainties for the two exposures which enter into the extrapolation to infinite density. The additional common uncertainty is $\pm 3 \%$. 


\subsection{Event classification}

The CHARM detector allows reconstruction of events and their classification as one-muon events $[\mathrm{N}(1 \mu)]$ and zero-muon events $[\mathrm{N}(0 \mu)]$ by computer code on an event-by-event basis. We have already used this technique of individual event classification to measure the NC-to-CC ratio of $\nu_{\mu}$ interactions [15].

Events with a muon in the final state are due to $\mathrm{CC} \nu_{\mu}$ and $\bar{\nu}_{\mu}$ interactions. The total energy for these events can be determined by combining the calorimetric shower measurement, which is possible for all events, with the muon momentum measurement, which is possible for most of the events. Since for most of the cases the charge of the muon can be determined by magnetic analysis, it is possible to distinguish between neutrino and antineutrino interactions. (Corrections due to the decay of pions and kaons are described in subsection 4.4). The efficiency for muon momentum measurement and charge determination is discussed in subsection 4.3 and the reconstruction of the $\nu_{\mu}$ energy spectra in subsection 4.5 .

Events without a muon in the final state are due to NC $\nu_{\mu}$ or $\nu_{\mathrm{e}}$ interactions and also, to CC $\nu_{\mathrm{e}}$ interactions. In addition, they contain CC $\nu_{\mu}$ interactions for which the muon was not found (for this correction, see subsection 4.4), and possibly also events from yet a different origin.

We applied two different methods to extract the spectra of promptly produced $\nu_{\mathrm{e}}$ from this $(0 \mu)$ sample. The first method consists in detecting $\mathrm{CC} \nu_{\mathrm{e}}$ interactions directly by selecting events which have an electromagnetic shower in the final state. This method is described in subsection 4.6. In this case, since $\nu_{\mu}$ and $\nu_{\mathrm{e}}$ interactions are measured independently, one can search for an excess in the $(0 \mu)$ sample which is not accounted for by interactions of $\nu_{\mathrm{e}}$ or $\nu_{\mu}$. This search will be discussed in subsection 7.5.

Such a search is not possible with the second method, because here we assume that the $(0 \mu)$ events originate only from NC interactions of $\nu_{\mu}$ or from $\nu_{\mathrm{e}}$ interactions (both $\mathrm{CC}$ and NC). Since the ratio of $\mathrm{NC}$ to $\mathrm{CC}$ interactions is well measured for $\nu_{\mu}[15]$, one can use the $\mathrm{CC}$ spectra to subtract the $\nu_{\mu} \mathrm{NC}$ component from the $(0 \mu)$ sample according to its shower energy.

None of the methods used to determine the $\nu_{\mathrm{e}}$ spectra allow one to distinguish between $\nu$ and $\bar{\nu}$. In both cases an assumption must therefore be made regarding the relative flux of $\nu_{\mathrm{e}}$ and $\bar{\nu}_{\mathrm{e}}$.

\subsection{Event reconstruction}

A filter program rejected events having the topology of cosmic-ray interactions, i.e. originating from particles entering the fiducial volume of the detector through the frames or through the back. We checked that no neutrino events fulfilling our acceptance criteria were rejected by this filter.

For the remaining events the reconstruction program determined the interaction vertex, the shower energy, and the direction of the shower. The program reconstructed all visible tracks and determined their momenta by magnetic analysis or range, or by a lower limit of the track momentum by range if the track left the detector. The muon charge was determined with sufficient precision by the magnetic analysis, i.e. if $\Delta \mathrm{p} / \mathrm{p}<100 \%$. Events with a track of at least $1 \mathrm{GeV}$ range or with a shower energy of at least $1.5 \mathrm{GeV}$ were retained for further analysis.

The events were classified as $(1 \mu)$ events if at least one track was found compatible with having originated from the vertex with an energy greater than $1 \mathrm{GeV}$, and a visible range outside the shower region of more than 3 absorption lengths, corresponding to a range of more than $0.67 \mathrm{GeV}$ or about 13 target plates (fig. 3). Events without such a track were classified as $(0 \mu)$ events.

Figure 9a shows the efficiency for finding low-energy muons, which was calculated using a semi-Monte Carlo method (see subsection 4.4). For those tracks which did not traverse a sufficient length of magnetized iron, the charge was not determined. The efficiency for measuring the charge, calculated by Monte Carlo simulation, is shown in fig. $9 \mathrm{~b}$ ). 


\subsection{Corrections to the data}

To correct for misclassification of events as well as for possibly wrong determinations of the lateral vertex position, we performed a visual scan of a fraction of the events. Two different data samples were scanned. One sample was determined by the analysis program. It contained all events for which the automatic event classification or the vertex position determination might have been in error due to events with unusual pattern or ambiguous topology: for example, events with low shower energy (between $1.5 \mathrm{GeV}$ and $5 \mathrm{GeV}$ ), and events where the muon track does not extrapolate to the shower vertex within given limits; events which have additional, parasitic tracks; and events with a vertex position close to a boundary of the fiducial volume. This sample contained $23 \%$ of all events.

In addition, a random sample of $10 \%$ of all events was scanned to check the computer code and to determine the uncertainty of classification. From these measurements we calculated scanning corrections applying to the full data set.

The final result of the scanning corrections was an increase by $2.1 \%$ (61 events) of the $(0 \mu)$ sample and a decrease by $5.0 \%$ (244 events) of the $(1 \mu)$ sample. The remaining uncertainty of the event classification can be represented by a standard deviation of $\sigma= \pm 0.3 \%$ for each event class.

The event numbers for the different categories found inside the fiducial volume with a shower energy $\mathrm{E}_{\mathrm{sh}} 2 \mathrm{GeV}$ are listed in table 5 as raw data. Scanning corrections have been already applied.

Neutrino parents in the residual gas of the decay tunnel (pressure $\approx 0.02$ Torr) and in the material between the decay tunnel and the front face of the target produce a background of neutrinos which would appear as prompt in the extrapolation to infinite density. We calculated a background of $0.63 \%$ of the conventional flux from the $\varrho=1$ target. We assumed that it is energy-independent and subtracted it from the data (table 5 , upstream material).

To correct for residual cosmic-ray background events not rejected by the reconstruction program, we recorded data when the accelerator was off. The analysis was performed in the same way as for the neutrino data, using the filter, event classification, and scanning. Cosmic-ray events which were accepted were normalized to the effective live-time of the experiment and subtracted from the data in bins of shower energy (fig. 10a and table 5):

A small fraction of the $\mathrm{NC}$ events contained a track which fulfilled the requirements of a primary muon. These events were classified as $(1 \mu)$ events. This background was caused by decays of pions and kaons in the shower or by non-interacting hadrons, the so-called punch-through tracks. The correction was evaluated by analysing $(1 \mu)$ events in which the primary muon track was removed. The fraction of events with another track which satisfied the muon recognition criteria yielded the correction. This procedure was applied in bins of hadron energy to take into account the difference in $\mathrm{NC}$ and $\mathrm{CC}$ hadron energy distributions. A small contribution, present in $\mathrm{CC}$ but not in NC events, was due to dimuons from charm-meson decays. This was calculated and subtracted. The correction for hadron energies above $2 \mathrm{GeV}$ increased the $(0 \mu)$ sample by $(2.1 \pm 0.6) \%$ (see fig. $10 \mathrm{~b}$ and table 5 ).

In a small fraction of the $\mathrm{CC}$ events the muon escaped detection. These were events with a muon energy of less than $1 \mathrm{GeV}$ (soft muons), with a muon leaving the detector with a visible track length corresponding to less than $1 \mathrm{GeV}$ of energy loss (side muons), or with a muon track which was obscured by the shower (hidden muons).

In the first case the fraction of lost events depends only on the muon momentum; in the second case it also depends on the angular distribution of the muons and on the geometry of the detector. As the space-time structure of the CC interaction and the nucleon structure functions are well known, the inefficiencies due to these effects can be reliably calculated using Monte Carlo techniques to simulate the $\mathrm{CC}$ events and to track the muons through the detector in order to determine the fraction of muons lost at its sides. 
To correct for hidden muons, a knowledge of the shape of the hadron showers and of the combined efficiency of the reconstruction program and of the visual scan is required. We therefore used in a semi-Monte Carlo method all $(1 \mu)$ events recorded in the beam-dump exposure, removed all reconstructed tracks which were candidates for leading muons, and then generated a new muon track by Monte Carlo techniques according to the known $\mathrm{x}$ and y distributions of CC $\nu_{\mu}$ and $\bar{\nu}_{\mu}$ events. These semi-Monte Carlo events were then analysed by the reconstruction program and scanned.

The global corrections determined in this way are given in table 5. The combined correction for muon losses is shown in fig. $10 \mathrm{c}$ as a function of the shower energy. The correction increases the number of $(1 \mu)$ events by $(7.5 \pm 0.5) \%$ and reduces the number of $(0 \mu)$ events by $(11.2 \pm 0.7) \%$. We thus arrived at the corrected number of events in table 5 , and the rates per tonne of fiducial volume and $10^{18}$ POT given in table 6 . The systematic errors quoted combine the errors of all corrections, the scanning and normalization errors discussed in the previous section, and, in addition, the error due to a $3 \%$ uncertainty of the energy scale at the cut of $2 \mathrm{GeV}$ corresponding to $\pm 0.4 \%$ of the rates. Using a Monte Carlo method, we combined these errors with the total systematic uncertainty which took known correlations into account and assumed Gaussian error propagation (see also subsection 4.7).

Corrected event rates in bins of energy are given in table 7.

\subsection{Determination of muon-neutrino spectra}

We applied a statistical unfolding procedure to determine separately the spectra of $\nu_{\mu}$ and $\bar{\nu}_{\mu}$. In this method the observed spectra $\mathrm{O}_{\mathrm{F}}\left(\mathrm{E}^{\prime}\right)$ are expressed as the convolution of the real spectra $\mathrm{I}(\mathrm{E})$ with a function $\mathrm{M}_{\mathrm{F}}\left(\mathrm{E}, \mathrm{E}^{\prime}\right)$ :

$$
\mathrm{O}_{\mathrm{F}}\left(\mathrm{E}^{\prime}\right)=\int \mathrm{M}_{\mathrm{F}}\left(\mathrm{E}, \mathrm{E}^{\prime}\right) \times \mathrm{I}(\mathrm{E}) \mathrm{dE}
$$

where $\mathrm{E}$ is the neutrino energy and $\mathrm{E}^{\prime}$ the total measured energy for the events. The function $M_{F}\left(E, E^{\prime}\right)$ contains the resolution functions for the measurement of the hadron shower energy and the muon momentum, as well as the acceptance functions which depend on the kinematics of the reactions (supposed to be known), on the detector geometry, and on the magnetic field configuration.

The method consists now in varying the real spectrum $I(E)$ such that the products $M_{F} \times I$ reproduce the measured spectra $\mathrm{O}_{\mathrm{F}}$.

The procedure is performed separately for the two different target densities. As input we use two different classes of events: i) Those for which the muon momentum and the muon charge are measured $(\Delta \mathrm{p} / \mathrm{p}<1)$. There are four of these spectra for each density, distinguished by the charge of the muon and the polarity of the magnetic field. ii) All events classified as CC interactions, i.e. comprising also those events where the charge of the muon and/or its momentum could not be measured. There are two of these spectra for each density, distinguished by the polarity of the magnetic field. The method uses the constraint that within the limit set by the statistical accuracy the resulting spectra for the two magnet polarities must be equal.

To cross-check the various correction procedures, we performed the same analysis starting from event samples that are separated into raw $(1 \mu)$ and $(0 \mu)$ events by the presence or absence of a muon track with a momentum of at least $5 \mathrm{GeV} / \mathrm{c}$. In this case cosmic-ray background appears as $(0 \mu)$ events only, the $\pi, \mathrm{K} \rightarrow \mu$ decay contribution becomes negligibly small, and the main correction is due to acceptance losses of soft and side muons. We selected, in addition, events with fitted muons only $(\Delta \mathrm{p} / \mathrm{p}<1)$ and performed a standard acceptance calculation.

The results of the two methods are compared in table 8 . The agreement is satisfactory and we consider the difference appearing in the number of events for $\varrho=1 / 3$ as an estimate of the systematic 
error inherent in the methods. Finally, we used the result from the acceptance calculation to determine the absolute rates, and the unfolding procedure to determine the shape of the spectra and the separation into $\nu$ and $\bar{\nu}$ events.

The resulting event rates in bins of energy are given in table 9 and shown in fig. 11. They have been used in the Monte Carlo calculation of the corrections for muon losses and $\pi, \mathrm{K} \rightarrow \mu$ decay described in subsection 4.4 .

In fig. 12 the inelasticity distributions in $\mathrm{y}=\mathrm{E}_{\text {hadron }} / \mathrm{E}_{\nu}$ are shown separately for events with a $\mu^{-}$and a $\mu^{+}$from the $\varrho=1$ target, and are compared with distributions calculated from the smoothed unfolded spectra which we expect for CC $\nu_{\mu}$ and $\bar{\nu}_{\mu}$ interactions. We note good agreement of the data with the expectation and therefore feel justified in assuming that all events with a muon are induced by $\mathrm{CC} \nu_{\mu}$ and $\bar{\nu}_{\mu}$ interactions.

In table 6 we also give the rates for different energy cuts to permit a comparison of our results with those from other experiments, which in general have more restricted energy acceptance (see refs. [1], [3], [5]-[7]).

\subsection{Detection of charged-current electron-neutrino interactions}

A direct separation of $\nu_{\mathrm{e}}$-induced $\mathrm{CC}$ events from all muonless events was performed by computer code. The same method was used in the previous beam-dump experiment [2] and is described also in refs. [10], [11], and [16]. In the case of $\nu_{\mathrm{e}^{-}}$and $\bar{\nu}_{\mathrm{e}}$-induced CC events, the shower detected in the calorimeter consists of two components: an electromagnetic part induced by the electron, and a hadronic part induced by the recoiling hadron system. In most cases these two components are not separated in space. To recognize an electron shower and to measure the energies of the electron and hadron showers separately, we made use of the narrow width ( $2.5 \mathrm{~cm}$ FWHM) and the regular longitudinal development of electron showers instead of the broader and irregular hadron showers (typically $20 \mathrm{~cm}$ FWHM). An electromagnetic shower was detected in a 'lane' of scintillators (15 cm wide) starting at the shower vertex and extending over a length chosen, according to the energy of the electron candidate, to contain approximately $95 \%$ of the total electron energy.

We used four different estimators to determine the energy of electron candidates. They consisted of the energy detected i) in the scintillator at the maximum of the shower development, ii) in three scintillators around and including the shower maximum in three adjacent planes, thus combining information from scintillators of horizontal and vertical orientation, iii) in all horizontally oriented scintillators of the lane, and iv) in all vertically oriented scintillators of the lane. These estimators are strongly correlated for electromagnetic showers.

During many trials, we searched for shower directions starting at or in a region close to the reconstructed interaction vertex. We retained, as the most probable candidate for an electromagnetic shower, the trial for which the energy estimators showed the best consistency.

The response and the resolution of the estimators were calibrated with data recorded in an electron beam for energies from $5 \mathrm{GeV}$ to $50 \mathrm{GeV}$ [10]. Monte Carlo generated showers were used to extend the calibration range up to $150 \mathrm{GeV}$. Figure 13 shows the result of applying the method to events induced by $15 \mathrm{GeV}$ pions and electrons in the test beam. The quantity y defined as

$$
y=\left(E_{\text {total }}-E_{\text {electromagnetic }}\right) / E_{\text {total }}
$$

is shown, where $E_{\text {electromagnetic }}$ is obtained from the weighted average of the four energy measurements and $E_{\text {total }}$ is the total shower energy. Applied to charged current $\nu_{\mathrm{e}}$ interactions, y corresponds to the inelasticity.

For CC $\nu_{\mathrm{e}}$ and $\bar{\nu}_{\mathrm{e}}$ events the response of the estimators was systematically shifted, and the resolution deteriorated owing to the presence of the hadronic shower in the volume covered by the 
search. We evaluated this effect by applying the method to semi-Monte Carlo events, which were constructed from $\mathrm{CC} \nu_{\mu}$ events recorded in the same exposure, replacing the muon by an electromagnetic shower, of the same energy and direction, generated by a Monte Carlo program.

The search was applied to all $(0 \mu)$ events of the beam-dump data. The resulting y-distributions are composed of two parts, one from genuine CC $\nu_{\mathrm{e}}$ and $\bar{\nu}_{\mathrm{e}}$ events, the other from a background of $\mathrm{NC}$ events for which, in general, there is also an electromagnetic component which is either due to a $\pi^{0}$ produced at the vertex, or exists simply because a part of the hadronic shower fulfils the criteria. These $y$-distributions were determined in bins of shower energy for the different target densities.

We separated the two components by a statistical analysis. The shape of the background distribution was determined by applying the method to NC events of the same energy distribution recorded in the wide-band beam using the same trigger condition. We mixed events obtained in the $\nu_{\mu}$ and $\bar{\nu}_{\mu}$ beams as expected for equal fluxes in the beam-dump experiment. The beam contains a small contamination of $\nu_{\mathrm{e}}$ and $\bar{\nu}_{\mathrm{e}}$ of about $2.0 \%$ on the average $[17,18]$, which is taken into account in the analysis. To determine the shape of the CC $\left(\nu_{\mathrm{e}}+\bar{\nu}_{\mathrm{e}}\right) \mathrm{y}$-distribution, we assumed for prompt $\nu_{\mathrm{e}}$ a flux ratio of $\bar{\nu}_{\mathrm{e}} / \nu_{\mathrm{e}}=1$ and folded, by Monte Carlo calculation, the y-distribution with the experimentally determined shift and resolution function for $\mathrm{y}_{\text {measured. }}$. The relative amount of the two components $\left(\nu_{\mathrm{e}}\right.$ $+\bar{\nu}_{\mathrm{e}}$ ) events and NC background, was then determined by a fit to the experimental distribution. The resulting y-distributions were found to agree well with the Monte Carlo predictions, as depicted in fig. 14. We show here as an example the analysis of $(0 \mu)$ data recorded with the $\varrho=1$ target in the energy range from 40 to $60 \mathrm{GeV}$.

We estimated the systematic uncertainty of the method by varying the parameters used in the analysis, within reasonable limits. The upper limit of the fit range in y was varied from 0.5 to 1.0 , the shift and resolution functions for the y measurement were modified, and the flux ratio of $\nu_{\mathrm{e}}$ and $\bar{\nu}_{\mathrm{e}}$ was varied from 0.4 to 1.4 .

Above $2 \mathrm{GeV}$ neutrino energy we found $530 \pm 49 \pm 40 \mathrm{CC} \nu_{\mathrm{e}}$ events at $\varrho=1$ and $342 \pm 44 \pm$ 26 events at $\varrho=1 / 3$. The energy dependence of the rates is given in table 10 .

\subsection{Systematic errors}

Systematic errors have already been mentioned in previous sections. In this subsection we summarize and complete the discussion of error sources. We explain the method applied to take into account the correlations and to evaluate the propagation of these errors through the various steps of the analysis.

We considered the following sources of systematic errors:

1) Event classification, where errors are mainly due to uncertainties of the vertex position: From the scanning procedure we derived an uncertainty of $\pm 0.3 \%$ in the observed events.

2) Shower energy calibration: The absolute calibration of the shower energy has an error of $\pm 3 \%$ [10]. This causes a variation of $\pm 0.4 \%$ in the number of events above an energy cut of $20 \mathrm{GeV}$.

3) Correction for cosmic-ray background: The error is $\pm 15 \%$ of the corrections applied to $(0 \mu)$ events and $\pm 40 \%$ of the corrections applied to $(1 \mu)$ events. It corresponds to the statistical error of the sample of events that results from analysing cosmic-ray triggers.

4) Decay of pions and kaons with a muon in the final state: The error is $\pm 27 \%$ of the correction, mainly due to the statistics of the events available for the correction.

5) Correction for soft, side, and hidden muons: The error is $\pm 10 \%$ of the correction, derived from variations in the input assumptions used in the Monte Carlo calculation.

6) Correction due to upstream material: The error is $\pm 8 \%$ of the correction from an estimated uncertainty in the amount of material and its effect on neutrino production. 
7) Normalization to POT: The error is $\pm 0.5 \%$ due to random fluctuations of BCT measurements, plus $\pm 0.5 \%$ due to imperfections in proton accounting in the analysis program, plus $\pm 0.8 \%$ due to errors in dead-time measurements.

8) Corrections due to leakage of hadrons out of the $\varrho=1 / 3$ target: The error is $\pm 9.1 \%$ of the corrections, derived from varying the assumptions used in the calculations.

9) Acceptance of $\nu_{\mu}$ and $\bar{\nu}_{\mu}$ events as a function of neutrino energy. From a comparison of the results from the unfolding procedure and standard acceptance calculation, and a variation of assumptions entering into the calculations, we estimate an error of $\pm 2.5 \%$ on the rates.

10) Error on the average ratio $\langle\mathrm{R}\rangle$ of $\mathrm{NC} / \mathrm{CC}$ for $\nu_{\mu}$ interactions. The error of $\pm 1.3 \%$ is a combination of the errors on the measurement of $\mathrm{R}$ and $\overline{\mathrm{R}}$ and the uncertainty in the relative neutrino to antineutrino flux in the present experiment.

11) Error in the direct determination of $\nu_{\mathrm{e}}$ interactions, $\pm 7.5 \%$ of the measured event rates, estimated by varying the assumptions of the analysis.

12) Error on the evaluation of conventional fluxes: The errors depend on the kind of neutrino considered and on the energy cut applied, e.g. $\pm 8.9 \%$ for the combined $\nu_{\mu}+\bar{\nu}_{\mu}$ flux with $\mathrm{E}_{\nu}>$ $20 \mathrm{GeV}$. The details are explained in section 5 .

13) Uncertainty in the density of the dump targets: We assume an uncertainty of $\pm 0.5 \%$ in the knowledge of the density of the $Q=1 / 3$ target.

In order to treat the propagation of these systematic errors for the various steps of the analysis, we assume that they can be represented by Gaussian distributions with standard deviations as given in the above list.

We used a Monte Carlo program to evaluate the final systematic errors on derived quantities. This program takes into account all known correlations of these errors. For example, the error on the evaluation of the number of protons affects, in a completely correlated way, the samples of $(1 \mu)$ and $(0 \mu)$ events, and the error on the correction for pion and kaon decay is anticorrelated for $(1 \mu)$ and $(0 \mu)$ events.

The method also gives automatically the asymmetric error bars that arise for combinations of rates (e.g. ratios of rates) leading to non-Gaussian errors.

\section{DETERMINATION OF CONVENTIONAL NEUTRINO FLUXES}

We applied two different methods to separate events induced by neutrinos from prompt and from conventional sources. One method is based on extrapolation to infinite density and the other on subtraction of the interactions induced by neutrinos of conventional origin. The first method depends on reliable monitors of the proton fluxes which hit the targets of two different densities, on accurate dead-time corrections, on the relative density of the targets, and on identical experimental conditions for the two target densities. The second method relies on accurate measurements of the muon fluxes in the shields, and on a good simulation of the hadron cascade in the dump target. A comparison of the results obtained by both methods constitutes therefore an essential check of the reliability of the experimental results.

The simulation program uses a multichain fragmentation model based on the dual parton model [19] adjusted to experimental data [20]. The transverse momentum distribution for directly produced particles follows ref. [21],

$$
\mathrm{N}\left(\mathrm{p}_{\perp}\right) \propto \mathrm{p}_{\perp} \exp \left[-3.3 \mathrm{p}_{\perp}^{2} /\left(\mathrm{GeV}^{2} / \mathrm{c}^{2}\right)\right]
$$

where we assumed an error of $10 \%$ in the exponent.

Neutrino and muon fluxes were calculated from the decay probability in the free decay path and the energy of all cascade particles, including strange mesons and hyperons. 
In tracking muons through the target, the catcher, and the iron shield, we simulated the energy loss due to ionization, bremsstrahlung, pair production, and nuclear interactions, using a Monte Carlo program which was developed at CERN [22] and which gives excellent agreement with experimental data. Multiple scattering was simulated [23] following the Molière formalism.

By normalizing the calculated rate of the production of non-prompt muons to the measured fluxes, we reduced the uncertainties for the calculation of the conventional neutrino fluxes. The uncertainty in this normalization, which is due to the uncertainties in the flux measurement, the determination of the density of the iron shield, and the calculation of the energy loss of muons in the iron, gives rise to an error of $\pm 6.5 \%$ in the conventional neutrino event rate for energies above $20 \mathrm{GeV}$.

In addition to this, we considered the following sources that contribute to the uncertainty of the calculated flux above $20 \mathrm{GeV}$ neutrino energy:

i) We used the world-average values of total cross-sections [24]:

$$
\sigma_{\nu, \mathrm{CC}} / \mathrm{E}=0.67 \times 10^{-38} \mathrm{~cm}^{2} \mathrm{GeV} \quad \text { and } \quad \sigma_{\bar{\nu}, \mathrm{CC}} / \mathrm{E}=0.34 \times 10^{-38} \mathrm{~cm}^{2} / \mathrm{GeV},
$$

for which we assumed an error of $\pm 5 \%$.

ii) We assumed to know the $\mathrm{K} / \pi$ ratio with a precision of $\pm 10 \%$, resulting in a $\pm 3 \%$ uncertainty on the neutrino fluxes.

iii) The $\pm 10 \%$ error in the exponent of the $\mathrm{p}_{\perp}$-distribution contributes to the flux uncertainty by $\pm 2.8 \%$.

iv) An energy-dependent error on the $\pi^{-} / \pi^{+}$and $\mathrm{K}^{-} / \mathrm{K}^{+}$ratios contributes $\pm 1.8 \%$ to the neutrino flux uncertainty.

Adding all these errors in quadrature results in a total uncertainty of $\pm 8.9 \%$ for the rate of conventional $\left(\nu_{\mu}+\vec{\nu}_{\mu}\right)$ events above $20 \mathrm{GeV}$ neutrino energy.

The uncertainties for an estimate of the conventional $\left(\nu_{\mathrm{e}}+\bar{\nu}_{\mathrm{e}}\right)$ event rate was obtained in a similar fashion. We assumed that the cross-sections are the same as those for muon neutrinos. The simulation is mainly sensitive to errors on the $\mathrm{K} / \pi$ ratio $( \pm 10 \%)$ and on the hyperon $/ \pi$ ratio ( $\pm 50 \%$ ), yielding a total systematic error of $\pm 13 \%$ for the conventional $\nu_{\mathrm{e}}$ flux and of $\pm 17 \%$ for the $\bar{\nu}_{\mathrm{e}}$ flux.

The results of the flux determination have been confirmed by calculations with another cascade simulation program [25] which uses a phenomenological approach based on a parametrization of pion and kaon production data $[26,27]$.

Figure 15 shows a comparison of the spectrum of events induced by conventional $\nu_{\mu}$ and $\bar{\nu}_{\mu}$, as determined from the extrapolation of the neutrino data and the spectrum obtained from the muon flux measurement and cascade simulation. We note good agreement. The rates of $\nu_{\mu}$ and $\nu_{\mathrm{e}}$ are summarized in table 11 .

\section{RESULTS}

We evaluated the prompt event rates by extrapolating to infinite target density as well as by subtracting the rate of conventional neutrino interactions.

To extrapolate the rates obtained from the $\varrho=1$ target $\left(R_{1}\right)$ and the $\varrho=1 / 3$ target $\left(R_{1 / 3}\right)$ to infinite density $\left(\mathrm{R}_{\infty}\right)$, we used the relation

$$
\mathrm{R}_{\infty}=0.5 \times\left(3 \times \mathrm{R}_{1}-\mathrm{R}_{1 / 3}\right)-\alpha / 2 \times\left(\mathrm{R}_{1 / 3}-\mathrm{R}_{1}\right)
$$


where $\alpha$ is the energy-dependent correction due to hadron leakage from the $\varrho=1 / 3$ target. This correction was discussed in detail in section 3. For $\mathrm{E}_{\nu}>20 \mathrm{GeV}$ we found $\alpha=(3.5 \pm 0.4) \%$. The effect of upstream material had already been subtracted (see subsection 4.4).

To determine the systematic errors on the prompt rates, we took into account the uncertainty of $0.5 \%$ on the density of the $\varrho=1 / 3$ target in addition to the systematic errors on the non-prompt rates that are discussed in subsection 4.7.

The alternative method, i.e. subtraction of the rate of events induced by conventional neutrinos in order to obtain the prompt rates, gives higher statistical accuracy but is subject to larger systematic errors. However, the precise measurement of muon fluxes used in the determination of these conventional rates has resulted in a reduction of these systematic uncertainties. We applied the subtraction method to the target $\varrho=1$ rates to test the internal consistency of the data samples and the analysis.

We compared these event rates with the results obtained by us in the 1979 experiment [2]. We corrected for the different solid angles subtended by the two experiments, assuming an $\mathrm{r}^{-2}$ distance dependence for the prompt fluxes. Taking into account the slightly different fiducial target masses, we found a total scaling factor of 3.02. As the angular distribution of the prompt neutrino flux is expected to be fairly flat, an $\mathrm{r}^{-2}$ law is a good approximation; but the neutrino fluxes originating from conventional sources are more peaked in the forward direction, and in this case the $\mathrm{r}^{-2}$ law is not a good approximation. We calculated the dilution factor of the conventional flux using the Monte Carlo simulation of the hadron cascade in the dump targets. The total scaling factors differ for events with and without muons, because of the different ratio of prompt to conventional fluxes.

The comparison of rates for events with $\mathrm{E}_{\mathrm{sh}}>2 \mathrm{GeV}$ is given in table 12. The rates of the 1979 experiment, calculated using a constant scaling factor or a scaling factor determined separately for prompt and conventional fluxes, differ only by about $4 \%$. The agreement with the results of the new experiment is excellent.

\subsection{Results on muon-neutrino-induced charged-current events}

The rates of events with a muon, $\mathrm{R}(1 \mu)$, induced by prompt $\nu_{\mu}$ and $\tilde{\nu}_{\mu}$, and by the sum of both, have been summarized in table 6 for three different threshold energies, $\mathrm{E}_{\nu}>2 \mathrm{GeV}, 20 \mathrm{GeV}$, and $80 \mathrm{GeV}$. The prompt rates were obtained by extrapolation and by subtraction of the conventional rates from the $\varrho=1$ target rate. We note consistency of the two methods within the error limits quoted.

The results with $\mathrm{E}_{\nu}>20 \mathrm{GeV}$ have smaller errors than the ones with a cut at $2 \mathrm{GeV}$ and were therefore used mainly in the following discussions. The energy dependence is given in fig. 16, together with the predictions of a model of hadronic production and semileptonic decay of charmed hadrons (see section 7).

\subsection{Results on muonless events}

The rates of muonless events $R(0 \mu)$ can be used to evaluate the prompt flux of $\nu_{\mathrm{e}}$ and $\bar{\nu}_{\mathrm{e}}$ under the assumption that all $(0 \mu)$ events are due to NC $\nu_{\mu}$ and $\bar{\nu}_{\mu}$ interactions and to $\nu_{\mathrm{e}}$ and $\bar{\nu}_{\mathrm{e}}$ interactions. The $\nu_{\mu}$-induced NC events are subtracted on the basis of the muonic events $\mathrm{R}(1 \mu)$. The ratio of the total cross-sections of $\mathrm{NC}$ and $\mathrm{CC}$ interactions is known from experiments [15]. We use $\left(\mathrm{E}_{\mathrm{sh}}>\right.$ $2 \mathrm{GeV}$ )

$$
\mathrm{R}=\sigma^{\mathrm{NC}}\left(\nu_{\mu}\right) / \varrho^{\mathrm{CC}}\left(\nu_{\mu}\right)=0.313 \pm 0.003
$$

and 


$$
\overline{\mathrm{R}}=\sigma^{\mathrm{NC}}\left(\bar{\nu}_{\mu}\right) / \sigma^{\mathrm{CC}}\left(\bar{\nu}_{\mu}\right)=0.395 \pm 0.014
$$

and evaluate an average $\langle R\rangle$ from the relation

$$
\langle\mathbf{R}\rangle=\left[\mathbf{R} \times \mathrm{N}\left(\mu^{-}\right)+\overline{\mathbf{R}} \times \mathrm{N}\left(\mu^{+}\right)\right] /\left[\mathrm{N}\left(\mu^{-}\right)+\mathrm{N}\left(\mu^{+}\right)\right] .
$$

Within errors we find the same result for both densities and the different energy cuts applied:

$$
\langle R\rangle=0.333 \pm 0.004
$$

The rates obtained by subtracting $\langle R\rangle \times R(1 \mu)$ from the muonless rates constitute the total $\nu_{\mathrm{e}}$ rate comprising $\mathrm{CC}$ and $\mathrm{NC} \nu_{\mathrm{e}}$ and $\bar{\nu}_{\mathrm{e}}$ interactions. The subtraction is performed for both densities in bins of shower energy. Figure 17 shows the prompt muonless rate obtained by binwise extrapolation of the rates obtained with this procedure.

We extracted the CC component using an unfolding method for which we assume equal flux of $\nu_{\mathrm{e}}$ and $\bar{\nu}_{\mathrm{e}}$ in accordance with $\mathrm{D} \overline{\mathrm{D}}$ production and semileptonic decay (see section 7 ). The relative response of the calorimeter to electrons and hadrons, on the average 1.17, and the energy resolution functions for the different types of showers [10] are taken into account. The fraction of CC events in the sample of muonless events that remains after the subtraction of the $\nu_{\mu}$ and $\bar{\nu}_{\mu}$ component is $0.770 \pm 0.005,0.84 \pm 0.01$, and $0.79 \pm 0.01$ for shower energies above $2 \mathrm{GeV}, 20 \mathrm{GeV}$, and $80 \mathrm{GeV}$, respectively.

The result of this unfolding procedure as applied to the extrapolated prompt rate is shown in fig. 18. In table 13 we give the rates for the various components for three different threshold energies, $2 \mathrm{GeV}, 20 \mathrm{GeV}$, and $80 \mathrm{GeV}$. The prompt rates obtained by extrapolation and subtraction of conventional background (which is much smaller than for the case of $\nu_{\mu}$ ) are also given. We note very good consistency of the results.

\subsection{Results on electron-neutrino-induced charged-current events}

Using the method described in subsection 4.6 we determined the rate $R(1 \mathrm{e})$ induced by $\left(\nu_{\mathrm{e}}+\bar{\nu}_{\mathrm{e}}\right)$ directly, independently of any assumptions on their production. Figure 19 shows the prompt spectrum obtained by subtracting the conventional fraction from the event rates measured at $Q=1$. In table 14 we summarize the (1e) rates obtained for different energy cuts. The prompt rates obtained from the two methods again show very good agreement.

\subsection{Results on prompt muon production}

The cascade Monte Carlo program described in section 5 was used to separate the measured prompt muon flux into two components:

i) muons originating from the electromagnetic decay of mesons and Bethe-Heitler production of muon pairs created by decay gammas and Drell-Yan pairs;

ii) muons from semileptonic charm decay and other sources.

A detailed description of the procedure is given elsewhere [28].

The total prompt muon flux above a threshold energy of about $40 \mathrm{GeV}$, i.e. those muons reaching the second gap in the iron shield, is $(476 \pm 13) \mu / 10^{7}$ POT. After correction for target leakage effects and subtraction of the contribution due to electromagnetic decays and Bethe-Heitler pairs, we found $(72 \pm 36) \mu / 10^{7}$ POT due to semileptonic weak decays of charmed mesons. The error is essentially due to systematic uncertainties of the simulation. 


\section{DISCUSSION OF THE RESULTS}

\subsection{Prompt neutrino rates}

The two different methods that we applied in order to extract information on the rates of prompt $\nu_{\mathrm{c}}$ interactions have lead to results that are consistent with being equal (see tables 13 and 14). From the direct determination of $\mathrm{CC} \nu_{\mathrm{e}}$ interactions, we have obtained for neutrino energies above $20 \mathrm{GeV}$ a rate of $3.13 \pm 0.32$ (stat.), \pm 0.30 (syst.), whereas from the indirect determination from the $(0 \mu)$ sample we get $3.19 \pm 0.22$ (stat.) \pm 0.14 (syst.) in units of events per tonne of detector material and $10^{18}$ POT. In both cases the result obtained by subtracting the conventional background from the observed rates at $\varrho=1$ gives the smallest errors since the background is only $15 \%$, and we believe that it can be determined with $15 \%$ precision (see table 11 ).

The equality of the two results indicates that there is not much room for interactions of particles other than $\nu_{\mu}$ or $\nu_{\mathrm{e}}$. We discuss this further in subsection 7.5.

In the case of $\nu_{\mu}$ the most accurate results for the prompt interaction rates are obtained by applying extrapolation to infinite density. For neutrino energies above $20 \mathrm{GeV}$ we obtain a rate of $4.83 \pm 0.82$ (stat.) \pm 0.38 (syst.) events per tonne of detector and $10^{18}$ POT (see table 6). From this event rate we can calculate the prompt neutrino flux if we assume equal fluxes for neutrinos and antineutrinos. For energies above $20 \mathrm{GeV}$ we find a total prompt flux of $(0.37 \pm 0.06) \times 10^{-7} \nu_{\mu}$, per $\mu \mathrm{sr}$ and incident proton, into the solid angle of $24.3 \mu \mathrm{sr}$ subtended by the detector. The average energy of these neutrinos is $50.9 \pm 5.4 \mathrm{GeV}$.

A flux of prompt single muons produced in high-energy proton collisions has been reported normalized to the number of pions produced [29]. For comparison with these experiments we quote the ratio of prompt $\nu_{\mu}$ to $\pi^{+}$with energy greater than $20 \mathrm{GeV}$ in the forward cone of our acceptance. Using our estimates of $\pi^{+}$production (see section 5) and correcting for cascade contributions to the prompt $\nu_{\mu}$ flux $(-15 \%)$, we find a ratio of $\nu_{\mu} / \pi^{+}=(0.82 \pm 0.13) \times 10^{-5}$

We have shown in subsection 4.5 that, by analysing the y-distributions of events with positive and negative muons, these events can be consistently interpreted as being due to CC $\nu_{\mu}$ and $\bar{\nu}_{\mu}$ interactions.

The observed rates of prompt muonless events and prompt events containing a muon or an electron are in good agreement with the results we obtained in the previous beam-dump experiment [2] (see also table 12).

\subsection{Comparison of prompt $\nu_{\mu}$ and $\bar{\nu}_{\mu}$ fluxes}

The experimental result for the ratio of prompt $\bar{\nu}_{\mu}$ to $\nu_{\mu}$ event rates is $0.49_{-0.12}^{+0.24}$ (stat.) \pm 0.03 (syst.) for neutrino energies above $20 \mathrm{GeV}$; correcting for the $\bar{\nu}_{\mu}$ and $\nu_{\mu}$ total cross-section ratio $\sigma_{\bar{\nu}} / \sigma_{\nu}$ $=0.507$ [24], we obtain a flux ratio of $0.96_{-0.24}^{+0.47}$ (stat.) \pm 0.05 (syst.). Expressed in terms of a flux asymmetry, we get

$$
\left(\nu_{\mu}-\bar{\nu}_{\mu}\right) /\left(\nu_{\mu}+\bar{\nu}_{\mu}\right)=(2 \pm 16 \pm 2) \%
$$

Our result is compatible with equal fluxes of $\bar{\nu}_{\mu}$ and $\nu_{\mu}$, in agreement with the result of our previous beam-dump experiment, which gave a flux ratio of $1.3_{-0.5}^{+0.6}[2]$.

Within the experimental errors the event ratio does not depend on the neutrino energy, as can be seen from fig. 20.

$\mathrm{BEBC}[6]$ and CDHS [7, 30] give asymmetries of $-0.09 \pm 0.11$ and $0.13 \pm 0.12 \pm 0.04$, respectively, and the FNAL beam-dump experiment [5] quotes a ratio of fluxes of $\bar{\nu}_{\mu} / \nu_{\mu}=1.12 \pm$ $0.24 \pm 0.17$. A CalTech-Stanford Collaboration [29] reported a $\mu^{-}$to $\mu^{+}$ratio of $1.3 \pm 0.3$ from a measurement of prompt single muons produced in a $400 \mathrm{GeV}$ proton-iron collision in a larger angular range, compatible with our measurement of the $\bar{\nu}_{\mu}$ to $\nu_{\mu}$ ratio. 


\subsection{Production of charmed hadrons}

The observed prompt neutrino production can be described in terms of associated production and semileptonic decay of charmed hadrons. We restrict our discussion to the simplest processes: $\bar{D} \bar{D}$ and $\Lambda_{\mathrm{c}} \overline{\mathrm{D}}$ production. The event rates, energy spectra, and the $\bar{\nu}_{\mu}$ to $\nu_{\mu}$ flux ratio are relevant to the understanding of the production of charmed hadrons in proton-nucleus collisions.

For our analysis we parametrized the cross-section for inclusive charm production in the form

$$
\mathrm{E}\left(\mathrm{d}^{3} \sigma / \mathrm{dxdp}_{\perp}^{2}\right) \propto\left(1-\left|\mathrm{x}_{\mathrm{F}}\right|\right)^{\mathrm{n}} \exp \left(-\mathrm{bp}_{\perp}\right)
$$

where $\mathrm{x}_{\mathrm{F}}=\mathrm{p}_{\|} / \mathrm{p}_{\max }$, and $\mathrm{E}, \mathrm{p}_{\perp}$, and $\mathrm{p}_{\|}$denote the energy and the transverse and longitudinal momenta of the charmed hadron. Semileptonic charm decay was described by the spectator model of Altarelli et al. [31], which includes QCD corrections.

In addition to the production of charmed hadrons in the primary interaction of $400 \mathrm{GeV}$ protons, secondary particles (protons and pions) in the hadronic cascade in the dump target also contribute to charmed hadron production. We used a Monte Carlo simulation to estimate the cascade production due to protons and pions. We find, for example, for $\mathrm{n}=5$ and $\mathrm{b}=2 \mathrm{GeV}^{-1}$ and for the angular acceptance of our detector, an additional contribution of $15 \%$ from secondary interactions.

In figs. 16 and 18 (subsections 6.1 and 6.2, respectively) and in fig. 19 (subsection 6.3) we compare the predictions of the simple $\mathrm{D} \overline{\mathrm{D}}$ model with the neutrino energy distribution of CC events, and in fig. 17 (subsection 6.2) with the shower energy distribution of $\mathrm{CC}$ and NC $\nu_{\mathrm{e}}$ interactions. The parameters of $\mathrm{n}=5$ and $\mathrm{b}=2 \mathrm{GeV}^{-1}$ yield satisfactory agreement with the data.

For the following discussion of cross-sections we use the prompt $\nu_{\mathrm{e}}$ spectrum as obtained by subtracting the conventional background from the $R(0 \mu)-\langle R\rangle \times R(1 \mu)$ rates. The details of this analysis can be found in ref. [28].

The $\Lambda_{\mathrm{c}}$ production would be expected to have, for the $\left(1-\left|\mathrm{x}_{\mathrm{F}}\right|\right)$ term, an exponent of $\mathrm{n}=1$ according to quark counting rules [32]. However, such a term would lead to a strong neutrino energy dependence of the event ratio $\bar{\nu}_{\mu} / \nu_{\mu}$, favouring $\nu_{\mu}$ over $\bar{\nu}_{\mu}$ production at large values of $\mathrm{E}_{\nu}$. There is no experimental evidence for such an energy dependence of this ratio, as can be seen in fig. 20 (subsection 7.2). A fit to the distributions allowing for a $\left(1-\left|\mathrm{x}_{\mathrm{F}}\right|\right)$ component in addition to $(1-$ $\left.\mid \mathrm{x}_{\mathrm{F}}\right)^{\mathrm{n}}$ yields an upper limit of $\sigma\left(\Lambda_{\mathrm{c}} \overline{\mathrm{D}}\right) \times \mathrm{BR}\left(\Lambda_{\mathrm{c}} \rightarrow \nu+\mathrm{X}\right)$ of $4.5 \%$ of the total prompt rate at $90 \%$ confidence.

We conclude that prompt neutrino production can be described by central $\mathrm{D} \overline{\mathrm{D}}$ production. A fit to the cross-section parametrization given above yields a value of $n=4.8 \pm 0.4$. Assuming a linear dependence of the cross-section on the atomic number $\mathrm{A}$ of the target $(\mathrm{A}=63.5$ for copper $)$, and correcting for $15 \%$ cascade contributions, we obtained a product of the cross-section and the average semileptonic branching ratio for $\mathrm{D}^{0}$ and $\mathrm{D}^{ \pm}$of

$$
\sigma(\mathrm{pCu} \rightarrow \mathrm{D} \overline{\mathrm{D}} \mathrm{X}) \times \mathrm{BR}\left[\mathrm{D}(\overline{\mathrm{D}}) \rightarrow \nu_{\mathrm{e}}\left(\bar{\nu}_{\mathrm{e}}\right) \mathrm{X}\right]=1.9 \pm 0.2 \text { (stat.) } \pm 0.2 \text { (syst.) } \mu \mathrm{b} \text { per nucleon, }
$$

where the systematic error includes an uncertainty of the $b$ parameter of $\pm 0.3 \mathrm{GeV}^{-1}$.

We used the same model of production and semileptonic decay to calculate the acceptance of muons into the arrays of solid-state detectors in the iron shield. This acceptance depends very little on the parameters used for the production cross-section owing to the large solid angle subtended by these detectors.

If we interpret the prompt muon flux obtained after subtraction of the component due to electromagnetic decay and to Bethe-Heitler and Drell-Yan pairs, as being due to charm decay, we obtain a product of cross-section times branching ratio for $\mathrm{D} \overline{\mathrm{D}}$ production of $1.3 \pm 0.7 \mu \mathrm{b}$ per 
nucleon. The error is mainly due to uncertainties in the data used as an input to the Monte Carlo calculation. The result is compatible with those obtained from prompt neutrino production.

We can compare our results with the one obtained by Ritchie et al. [29]. They investigated prompt single-muon production in $350 \mathrm{GeV}$ proton-iron interactions. Their result, scaled by $20 \%$ to take into account the higher proton energy of our measurement [33], is $\sigma \times \mathrm{BR}\left[\mathrm{D}(\overline{\mathrm{D}}) \rightarrow \nu_{\mu}\left(\bar{\nu}_{\mu}\right) \mathrm{X}\right]=$ $2.2 \pm 0.2$ (stat.) \pm 0.3 (syst.) $\mu$ b per nucleon, in agreement with our results.

\subsection{Comparison of prompt electron and muon event rates}

The rate of prompt CC $\nu_{\mathrm{e}}$ interactions, given in tables 13 and 14, can be compared with the rate of prompt CC $\nu_{\mu}$ interactions of table 6 . The ratios and asymmetries obtained by combining the rates which have been determined by different methods and for different cuts in energy are given in table 15.

It has been customary to quote the ratio of fluxes, although the errors are asymmetric and non-Gaussian. We prefer to quote the main result of this experiment as an asymmetry. Combining the various results of table 15 we get for neutrino energies above $20 \mathrm{GeV}$,

$$
\mathrm{A}(\mathrm{e}, \mu)=\left(\mathrm{N}_{\nu_{\mu}}-\mathrm{N}_{\nu_{\mathrm{e}}}\right) /\left(\mathrm{N}_{\nu_{\mu}}+\mathrm{N}_{\nu_{\mathrm{e}}}\right)=(20 \pm 10 \pm 5) \%
$$

The corresponding flux ratio is $0.67 \pm 0.15$ (stat.) \pm 0.07 (syst.), which is not incompatible with the result of our previous experiment: $0.48 \pm 0.12$ (stat.) \pm 0.10 (syst.), or, translating the symmetric error quoted in our previous publication into intervals of $68 \%$ confidence, $0.48 \pm 0.09$ (stat.) $\pm_{0.09}^{0.13}$ (syst. $)^{*}$.

Prompt events of $\nu_{\mu}$ and $\nu_{\mathrm{e}}$ have a very similar energy distribution. For events above $20 \mathrm{GeV}$ we found the mean energies $\left\langle\mathrm{E}_{\nu_{\mu}}\right\rangle=70.8 \pm 6.6 \mathrm{GeV}$ and $\left\langle\mathrm{E}_{\nu_{\mathrm{e}}}\right\rangle=71.6 \pm 3.7 \mathrm{GeV}$. We did not find a significant variation of the asymmetry with energy as can be seen from fig. 21 .

An asymmetry of zero, up to small corrections of the order of some per cent [34], is predicted by the conventional e- $\mu$ universality of weak interactions. Our result is consistent with this prediction within the limits of statistical and systematic errors. This is also the case for other recent beam-dump results, which measure asymmetries of $(2.7 \pm 6.4 \pm 4.0) \%[5]$ and $(-7 \pm 8) \%[6]$ and $(7.8 \pm 7.1 \pm$ $3.8) \%[7,30]$. The weighted average of the four experiments yields an asymmetry of $(3.9 \pm 4.2) \%$ (statistical and systematic errors combined quadratically), compatible with zero. The results can be compared with the values of charm-decay branching ratios [24] for $\mathrm{D}^{+} \mathrm{D}^{0} \rightarrow(\mu+\mathrm{X})=(8.4 \pm$ $1.7) \%$ and $\mathrm{D}^{+} \mathrm{D}^{0} \rightarrow(\mathrm{e}+\mathrm{X})=(11.0 \pm 1.1) \%$, from which one can obtain an asymmetry of $\mathrm{A}=$ $(-13.4 \pm 11.1) \%$, again compatible with zero.

These asymmetry results support the hypothesis of $e-\mu$ universality in the decay of charmed particles.

\subsection{Excess of muonless events and search for $\nu_{\mathrm{X}}$ and $\nu_{\tau}$ interactions}

The rate of prompt muonless events $\mathrm{R}(0 \mu)$ was used already to evaluate the prompt $\nu_{\mathrm{e}}$ and $\bar{\nu}_{\mathrm{e}}$ flux (subsection 6.2). For that purpose we assumed that all muonless events are due to $\nu_{\mu}$ and $\bar{\nu}_{\mu} \mathrm{NC}$ interactions and to $\nu_{\mathrm{e}}$ and $\bar{\nu}_{\mathrm{e}} \mathrm{CC}$ and $\mathrm{NC}$ interactions.

*) We can also compare prompt rates of $\nu_{\mathrm{e}}$ and $\nu_{\mu}$ if we apply a cut of $>2 \mathrm{GeV}$ in only the hadron energy. In this case an acceptance calculation for muons (beyond the corrections of table 5) is not needed and the $\nu_{\mu}$ rates are those given in table 6 . The $\nu_{\mathrm{e}}$ signal is derived from the $\left(0_{\mu}\right)$ events by subtracting the $\nu_{\mu}$ contribution. In addition, a small correction has to be applied to subtract events with $\mathrm{E}_{\mathrm{sh}}>2 \mathrm{GeV}$ but $\mathrm{E}_{\mathrm{h}}<2 \mathrm{GeV}$. (See also subsection 7.5.) The resulting asymmetry is $\mathrm{A}=(12.5 \pm 11.5 \pm 9.0) \%$, compatible with the result obtained with the neutrino energy cut. 
We now ask the question, Is there any excess of muonless events which cannot be attributed to these interactions? Interactions of $\nu_{\tau}$ or of other unknown neutrino species $\left(\nu_{\mathrm{X}}\right)$ could give rise to events without an electron or a muon in the final state.

We have searched for such events assuming universality of $\nu_{\mathrm{e}}$ and $\nu_{\mu} \mathrm{CC}$ and $\mathrm{NC}$ interactions and by subtracting their contribution from $\mathbf{R}(0 \mu)$ :

$$
\mathrm{R}\left(\nu_{\mathrm{X}}\right)=\mathrm{R}(0 \mu)-\langle\mathrm{R}\rangle \times \mathrm{R}(1 \mu)-\left(1+\alpha \mathrm{R}^{\mathrm{e}}\right) \times \mathrm{R}(1 \mathrm{e}),
$$

where $\mathrm{R}^{\mathrm{e}}=0.340$ is the ratio of $\nu_{\mathrm{e}}$ and $\bar{\nu}_{\mathrm{e}} \mathrm{NC}$ and CC interactions assuming equal flux of $\nu_{\mathrm{e}}$ and $\bar{\nu}_{\mathrm{e}}$ [15]; $\alpha=\mathrm{R}\left[(1 \mathrm{e}), \mathrm{E}_{\mathrm{h}}>2 \mathrm{GeV}\right] / \mathrm{R}\left[(1 \mathrm{e}), \mathrm{E}_{\mathrm{sh}}>2 \mathrm{GeV}\right]=0.865 \pm 0.025$ was determined by Monte Carlo simulation.

Using all events with $\mathrm{E}_{\mathrm{sh}}>2 \mathrm{GeV}$ (see tables 13 and 14) we found $\mathrm{R}\left(\nu_{\mathrm{X}}\right)=27 \pm 84$ events per tonne and $10^{20}$ POT, compatible with zero. At $90 \%$ confidence level this corresponds to less than $21 \%$ of all prompt muonless events.

To interpret this excess for a limit on $\nu_{\tau}$ interactions, we evaluated by Monte Carlo methods the suppression of $\nu_{\tau} \mathrm{CC}$ interactions because of the threshold for $\tau$ production, and found $81 \%$ as compared to $\nu_{\mu}$ and $\nu_{\mathrm{e}}$ interactions. Because of the decay modes $\tau \rightarrow \mu \nu \bar{\nu}$ and $\tau \rightarrow \mathrm{e} \nu \bar{\nu}$ with $17.5 \%$ branching ratio [24], only $65 \%$ of the $\nu_{\tau} \mathrm{CC}$ interactions would be classified as muonless and electronless. Taking these modifications into account, we found $\operatorname{R}_{\mathrm{CC}}\left(\nu_{\gamma}\right)=28 \pm 87$ events per tonne and $10^{20}$ POT. At $90 \%$ confidence level this corresponds to a $\nu_{\tau}+\bar{\nu}_{\tau}$ flux of less than $21 \%$ of the total prompt flux. The BEBC experiment [35] has given a $\nu_{r}$ flux limit of $13 \%$.

In the 1979 experiment the number of $\nu_{\mathrm{e}}$ - and $\bar{\nu}_{\mathrm{e}}$-induced events to be subtracted from $\mathrm{R}(0 \mu)$ had been evaluated with the help of the charm production model which was normalized to the data for $\mathrm{E}_{\mathrm{sh}}>20 \mathrm{GeV}$. There is no a priori reason for expecting an excess for $\mathrm{E}_{\mathrm{sh}}<20 \mathrm{GeV}$. We therefore now normalized the prediction of the model to the whole energy range and determined the $\chi^{2}$ value for a deviation of our measured distribution from the expected one; we found a value of 0.85 per degree of freedom and hence no indication of a deviation from the model prediction. This normalization is shown in fig. 17.

\subsection{Verification of the universality of the weak neutral-current coupling}

The notion of universality of the coupling of the electron, the muon, and the tau family to the weak $\mathrm{CC}$ and $\mathrm{NC}$ is based mainly on experimental data [36]. The most precise comparison, at low values of $\mathrm{Q}^{2}$, of the relative coupling strength of the electron and muon families to the weak $\mathrm{CC}$ has been derived from precise measurements of the ratio of the corresponding pion decays, $\pi \rightarrow \mathrm{e} \nu_{\mathrm{e}}$ and $\pi \rightarrow \mu \nu_{\mu}$, with the result [37]

$$
\mathrm{g}_{\mathrm{e} \mathrm{e}_{\mathrm{e}}} / \mathrm{g}_{\mu \nu_{\mu}}=0.9939 \pm 0.0057
$$

From measurements of $\nu_{\mu}$ electron scattering $[18,38]$ and of the forward-backward asymmetry in the reaction $\mathrm{e}^{+} \mathrm{e}^{-} \rightarrow \mu^{+} \mu^{-}$[39] the relative axial-vector coupling strength of the electron and the muon to the weak NC have been derived, with the result

$$
\mathrm{g}_{\mathrm{ee}} / \mathrm{g}_{\mu \mu}=0.96 \pm 0.11
$$

No measurements with comparable accuracy have been reported for the relative coupling strengths of $\nu_{\mathrm{e}}$ and $\nu_{\mu}$ to the weak NC. These can be derived from measurements of the ratios of total cross-sections for semileptonic neutrino scattering, 


$$
\mathrm{R}^{\mathrm{e}}=\sigma\left(\nu_{\mathrm{e}} \mathrm{N} \rightarrow \nu_{\mathrm{e}} \mathrm{X}\right) / \sigma\left(\nu_{\mathrm{e}} \mathrm{N} \rightarrow \mathrm{eX}\right)
$$

and

$$
\mathrm{R}^{\mu}=\sigma\left(\nu_{\mu} \mathrm{N} \rightarrow \nu_{\mu} \mathrm{X}\right) / \sigma\left(\nu_{\mu} \mathrm{N} \rightarrow \mu \mathrm{X}\right)
$$

Values for $\mathrm{R}^{\mu}$ have been reported elsewhere [15]. In a beam composed of equal fluxes of $\nu_{\mu}$ and $\bar{\nu}_{\mu}$ we expect $R^{\mu}=0.340 \pm 0.005$ for the energy of the hadronic shower $E_{h}$ greater than $2 \mathrm{GeV}$ (see also subsection 6.2).

The equation used in subsection 7.5 to determine $\mathrm{N}\left(\nu_{\mathrm{X}}\right)$ can be used to determine $\mathrm{R}^{\mathrm{e}}$ if we assume that only $\nu_{\mathrm{e}}$ and $\nu_{\mu}$ contribute to the events in our detector, i.e. $\mathrm{N}\left(\nu_{\mathrm{X}}\right)=0$. Using the events obtained for both target densities we find [40]

$$
\mathrm{R}^{\mathrm{e}}=0.406_{-0.135}^{+0.145}
$$

Together with the value for $\mathrm{R}^{\mu}$ and assuming that $\mathrm{g}_{e \nu_{\mathrm{e}}} / \mathrm{g}_{\mu \nu_{\mu}}=1$, in agreement with the results from $\pi$ decay, we get

$$
\mathrm{g}_{\nu_{\mathrm{e}} \overline{\mathrm{v}}_{\mathrm{e}}} / \mathrm{g}_{\nu_{\mu}, \bar{\nu}_{\mu}}=\left(\mathrm{R}^{\mathrm{e}} / \mathrm{R}^{\mu}\right)^{1 / 2}=1.09_{-0.20}^{+0.18}
$$

We can combine this value with a result that we obtained from an exposure of our detector to a $160 \mathrm{GeV}$ narrow-band beam [41] and obtain the final result,

$$
\mathrm{g}_{\nu_{\mathrm{e}} \bar{\nu}_{\mathrm{e}}} / \mathrm{g}_{\nu_{\mu} \bar{\nu}_{\mu}}=1.05 \pm 0.15
$$

\subsection{Limit on the branching ratio $\pi^{0} \rightarrow \nu \bar{\nu}$}

The decay of $\pi^{0}$ into $\nu \bar{\nu}$ is forbidden for massless neutrinos. The observation of such a decay mode would imply the existence of massive neutrinos. It is therefore of interest to improve on the present limit of the branching ratio, which is $\mathrm{BR}\left(\pi^{0} \rightarrow \nu \bar{\nu}\right)<2.4 \times 10^{-5}[24,42]$.

Neutral pions decaying into a pair of neutrinos would contribute to the prompt neutrino flux in the detector. We can set an upper limit on the branching ratio by attributing to this $\pi^{0}$ decay mode all prompt neutrinos that cannot be accounted for by charm decay. A prediction of the prompt neutrino flux from charm decay can be derived from measurements of prompt muon production in proton-iron collision to which the $\pi^{0}$ decay does not contribute.

The production rate of $\pi^{0}$ is calculated with the Monte Carlo program described in section 5 . Including cascade production we obtain a production rate of $\pi^{0}$ with energies above $2 \mathrm{GeV}$ of $(19 \pm 4) \times 10^{-3} \pi^{0} / \mu \mathrm{sr} \cdot$ POT. Taking into account the acceptance of our detector for decay neutrinos, we expect to see $(4.3 \pm 0.8) \times 10^{5}$ neutrino events with an energy above $2 \mathrm{GeV}$ per tonne and $10^{18}$ POT for a branching ratio of 1 . For neutrino energies above $20 \mathrm{GeV}$ the expected rate is $(3.8 \pm 0.8) \times 10^{5}$ events per tonne and $10^{18}$ POT.

The production of proton-muons in proton-iron collisions was measured and interpreted in terms of $\mathrm{D} \overline{\mathrm{D}}$ production and decay by Ritchie et al. [29]. We can use the parametrization of charm production as given in [29] to calculate the expected flux of neutrinos in our detector, taking into account the higher proton energy. A signal that can be attributed to $\pi^{0}$ decay is obtained by subtracting this predicted flux from our measured prompt $\nu_{\mu}$ rate; we obtain a limit of $\mathrm{BR}\left(\pi^{0} \rightarrow \nu_{\mu}+\bar{\nu}_{\mu}\right)<7.8 \times 10^{-6}$. Assuming the semileptonic branching ratio for charm decay to be equal for decays into $\nu_{\mu}$ and $\nu_{\mathrm{e}}$, our measurement of the prompt $\nu_{\mathrm{e}}$ flux yields $\mathrm{BR}\left(\pi^{0} \rightarrow \nu_{\mathrm{e}}+\bar{\nu}_{\mathrm{e}}\right)<$ $1.7 \times 10^{-6}$. 
Our limit on $\nu_{T}$ interactions given in subsection 7.5 can be directly used to derive the limit $\operatorname{BR}\left(\pi^{0} \rightarrow \nu_{\tau}+\bar{\nu}_{\tau}\right)<4.1 \times 10^{-6}$.

The BEBC limit on $\nu_{\tau}$ production [35] can be used to obtain a limit of $\operatorname{BR}\left(\pi^{0} \rightarrow \nu_{\tau} \bar{\nu}_{\tau}\right)<2.3 \times$ $10^{-6}$.

Combining our results on decay into $\nu_{\mu} \bar{\nu}_{\mu}$ and $\nu_{\mathrm{e}} \bar{\nu}_{\mathrm{e}}$ with the limit derived from the BEBC result on $\nu_{\tau}$ production, we can set a general upper limit of

$$
\mathrm{BR}\left(\pi^{0} \rightarrow \nu+\bar{\nu}\right)<6.5 \times 10^{-6}
$$

All limits are given for a confidence level of $90 \%$.

\section{SUMMARY}

We have measured the production of prompt neutrinos in proton-copper collisions at an energy of $400 \mathrm{GeV}$ using the beam-dump technique. With respect to earlier experiments performed at CERN, the systematic uncertainties are reduced by improvements in the target arrangement and the beam-guidance and monitoring equipment.

The experiment has been performed with higher statistics so that our main results could be obtained by extrapolation to infinite density using different targets. We found consistent results by applying the subtraction method, for which the non-prompt neutrino flux is derived from measurements of the muon rates in the iron shield behind the dump target, which introduces some additional systematic uncertainties.

The CHARM detector allows the direct identification of CC $\nu_{\mu}$ and $\nu_{\mathrm{e}}$ interactions. For $\nu_{\mu}$ we can distinguish also between neutrino and antineutrino interactions at a high level of confidence.

Assuming universality of the electroweak coupling and the absence of interactions not induced by $\nu_{\mu}$ and $\nu_{\mathrm{e}}$, we can derive the flux of $\nu_{\mathrm{e}}$ also from the sample of muonless events. The result is consistent with the one obtained by direct electron identification.

We found the prompt fluxes to be equal for $\nu_{\mu}$ and $\bar{\nu}_{\mu}$ over the whole energy range. The asymmetry of the integrated fluxes is $\left[\Phi\left(\nu_{\mu}\right)-\Phi\left(\bar{\nu}_{\mu}\right)\right] /\left[\Phi\left(\nu_{\mu}\right)+\Phi\left(\bar{\nu}_{\mu}\right)\right]=0.02 \pm 0.16 \pm 0.02$.

The energy dependence of the prompt fluxes of all the neutrino species is consistent with them having originated from the production and semileptonic decay of charmed hadrons. A simple $\mathrm{D} \overline{\mathrm{D}}$ production model describes the data well. For the product of cross-section times branching ratio into $\nu_{\mathrm{e}}$, we obtained a value of $(1.9 \pm 0.2 \pm 0.2) \mu \mathrm{b}$ per nucleon, assuming the cross-section to be proportional to the atomic number $\mathrm{A}$ of the target. The dependence on the Feynman variable $\mathrm{x}$ is well described by the expression $\left(1-\left|\mathrm{x}_{\mathrm{F}}\right|\right)^{4.8 \pm 0.4}$, and charmed baryon production contributes less than $4.5 \%$ to the total prompt neutrino rate.

Comparing interaction rates of $\nu_{\mathrm{e}}$ and $\nu_{\mu}$, we found an asymmetry of $[\mathrm{R}(1 \mu)-\mathrm{R}(1 \mathrm{e})] /[(\mathrm{R}(1 \mu)+$ $\mathrm{R}(1 \mathrm{e})]=0.20 \pm 0.10 \pm 0.05$. This asymmetry is different from zero by less than two standard deviations and therefore does not exclude a value of zero as predicted for the decay of charmed hadrons. The weighted average asymmetry of four recent beam-dump experiments [5-7] is (3.9 \pm $4.2) \%$, in agreement with $e-\mu$ universality of charm decay.

We found no significant excess of leptonless events and hence no indication of neutrino interactions other than those induced by $\nu_{\mu}$ and $\nu_{\mathrm{e}}$. From this we derived a limit on the $\nu_{\tau}$ flux of less than $21 \%$ of the total prompt flux, and the total number of events that can possibly be attributed to interactions of an unknown kind of neutrino is less than $21 \%$ of all prompt muonless events, fully compatible with zero for the two cases.

All our results on the production of prompt neutrinos in proton-copper interactions are consistent with the assumption that they originate from charm production and decay. 
The high contribution of $\nu_{\mathrm{e}}$ interactions to the data sample was used to test the universality of the weak NC coupling of $\nu_{\mathrm{e}}$ and $\nu_{\mu}$. We found $\mathrm{g}_{\nu_{\mathrm{e}} \bar{\nu}_{\mathrm{e}}} / \mathrm{g}_{\nu_{\mu} \bar{\nu}_{\mu}}=1.05_{-0.18}^{+0.15}$, in agreement with universality.

Our results on the production of prompt neutrinos can be used to derive an improved limit on the decay of $\pi^{0}$ - which are copiously produced in the dump target -into neutrino-antineutrino pairs. Subtracting the prompt neutrino flux due to charmed hadron production and decay, and combining our result with the BEBC limit on $\nu_{\tau}$ production [35], we obtained $\mathrm{BR}\left(\pi^{0} \rightarrow \nu \bar{\nu}\right)<6.5 \times$ $10^{-6}$ at $90 \%$ confidence level.

\section{Acknowledgements}

We wish to thank the members of the group of $W$. Middelkoop and P. Weiss for the construction of the dumps, W. Kalbreyer for the design of the beam optics and the beam monitoring equipment, and for his continuous help during the operation of the beam; and the SPS staff for the operation of the accelerator. We gratefully acknowledge the skilful help of our many technical collaborators, and we also thank C. Busi and J. Audier for their help during data reduction and analysis. 


\section{REFERENCES}

[1] P. Fritze et al. (ABCLOS Collab.), Phys. Lett. 96B (1980) 427.

[2] M. Jonker et al. (CHARM Collab.), Phys. Lett. 96B (1980) 435.

[3] H. Abramowicz et al. (CDHS Collab.), Z. Phys. 13C (1982) 179.

[4] K. Winter, Proc. Int. Symp. on Lepton and Photon Interactions at High Energies, Cornell, 1983, eds. D.G. Cassel and D.L. Kreineck (F.R. Newman Lab., Cornell Univ., Ithaca, NY, 1983), p. 177.

F. Bergsma et al. (CHARM Collab.), Proc. 12th Int. Conf. on Neutrino Physics and Astrophysics, Sendai, 1986, eds. T. Kitagaki and H. Yuta (World Scientific, Singapore, 1986), p. 402.

[5] R.C. Ball et al., Phys. Rev. Lett. 51 (1983) 743.

M.E. Duffy et al., Phys. Rev. Lett. 52 (1984) 1865, 55 (1985) 1816, and 57 (1986) 1522.

[6] H. Grässler et al., Nucl. Phys. B273 (1986) 253.

[7] P. Perez (CDHS Collab.), Proc. Int. Europhysics Conference on High-Energy Physics, Brighton, 1983 (RAL, Didcot, 1983), p. 316.

[8] F. Bergsma et al. (CHARM Collab.), Phys. Lett. 157B (1985) 458 and 166B (1986) 473.

[9] A.N. Diddens et al. (CHARM Collab.), Nucl. Instrum. Methods 178 (1980) 27.

[10] J. Dorenbosch et al. (CHARM Collab.), Nucl. Instrum. Methods A253 (1987) 203.

[11] M. Jonker et al. (CHARM Collab.), Nucl. Instrum. Methods 215 (1983) 361.

[12] E.H.M. Heijne, CERN 83-06 (1983).

[13] V. Goldansky, A. Kutsenko and M. Podgoretsky, Statistics of nuclear particle counting (Physics-Mathematics Publishing House, Moscow, 1959).

[14] A.G. Frodesen, O. Skjeggestad and H. Tofte, Probability and statistics in particle physics (Universitetsforlaget, Bergen, 1979).

[15] M. Jonker et al. (CHARM Collab.), Phys. Lett. 99B (1981) 265.

J.V. Allaby et al. (CHARM Collab.), Phys. Lett. 177B (1986) 446 and Z. Phys. C36 (1987) 611.

[16] C.H.M. Nieuwenhuis, Thesis, Amsterdam (1986).

[17] M. Jonker et al. (CHARM Collab.), Phys. Lett. 107B (1981) 241.

[18] F. Bergsma et al. (CHARM Collab.), Phys. Lett. 147B (1984) 481.

[19] P.A. Arnio, J. Ranft and G.R. Stevenson, CERN/TIS-RP/106/Rev. (1984).

J. Ranft and S. Ritter, CERN/TIS-RP/103/PP (1983).

[20] J. Ranft and S. Ritter, CERN/TIS-RP/137/PP (1984).

H.J. Möhring, CERN/TIS-RP/116 (1983).

[21] H. Kirk et al., Nucl. Phys. B128 (1977) 397.

[22] W. Lohmann, R. Kopp and R. Voss, CERN 85-03 (1985).

[23] CERN Computer Centre Program Library, Routine MLR.

[24] Particle Data Group, Review of Particle Properties, Phys. Lett. 170B (1986) 1.

[25] A. Baroncelli, Nucl. Instrum. Methods 118 (1974) 445.

[26] H. Atherton et al., CERN 80-07 (1980).

[27] J. Eichten et al., Nucl. Phys. B44 (1972) 333.

[28] F. Bergsma, Thesis, Amsterdam (1988).

[29] J.L. Ritchie et al., Phys. Lett. 126B (1983) 499.

[30] F. Dydak (CDHS Collab.), private communication.

[31] G. Altarelli et al., Nucl. Phys. B208 (1982) 365. 
[32] R.T. Edwards et al., Phys. Rev. D18 (1978) 76.

[33] R. Odorico, Nucl. Phys. B209 (1982) 77.

[34] E.L. Berger, L. Clavelli and N.R. Wright, Phys. Rev. D27 (1983) 1080.

[35] M. Talebzadeh et al., Nucl. Phys. B291 (1987) 503.

[36] J.D. Bjorken and S. Weinberg, Phys. Rev. Lett. 38 (1977) 622.

[37] E. di Capua et al., Phys. Rev. B133 (1964) 1333.

D.A. Bryman et al., Phys. Rev. D11 (1975) 1337 and Phys. Rev. Lett. 50 (1983) 7.

[38] L.A. Ahrens et al., Phys. Rev. Lett. 55 (1985) 1814.

[39] See, for example, B. Naroska, Proc. Int. Symp. on Lepton and Photon Interactions at High Energies, Ithaca, 1983, eds. D.G. Cassel and L. Kreinick (Cornell Univ., Ithaca, NY, 1983), p. 96.

[40] J.V. Allaby et al. (CHARM Collab.), Phys. Lett. 180B (1986) 303.

[41] J.V. Allaby et al. (CHARM Collab.), Phys. Lett. 179B (1986) 301.

[42] P. Herczeg and C.M. Hoffman, Phys. Lett. 100B (1981) 347. 
Table 1

Main parameters of the experimental set-up

\begin{tabular}{|c|c|c|}
\hline Proton beam & $\begin{array}{l}\text { Energy } \\
\text { Spill-time } \\
\text { Repetition cycle } \\
\text { Typical intensity }\end{array}$ & $\begin{array}{l}400 \mathrm{GeV} \\
23 \mu \mathrm{s} \\
\sim 10 \mathrm{~s} \\
10^{13} \mathrm{ppp}\end{array}$ \\
\hline Dump target & $\begin{array}{l}\text { Dimension } \\
\text { Density } \varrho=1 \\
\text { Density } \varrho=1 / 2\end{array}$ & $\begin{array}{l}41.5 \times 31 \times 302.5 \mathrm{~cm}^{3} \\
\text { Solid copper } 8.96 \mathrm{~g} / \mathrm{cm}^{3} \\
2.5 \mathrm{~cm} \text { copper } / 2.5 \mathrm{~cm} \text { air } \\
\text { over } 2 / 3 \text { of the total length } \\
2.5 \mathrm{~cm} \text { copper } / 5 \mathrm{~cm} \text { air } \\
\text { over the total length }\end{array}$ \\
\hline Distance & $\begin{array}{l}\text { Upstream end of target to } \\
\text { centre of fiducial volume }\end{array}$ & $487.3 \mathrm{~m}$ \\
\hline Fiducial volume & $\begin{array}{l}\text { Mass } \\
\text { Cross-section } \\
\text { Solid angle } \\
\text { Average density }\end{array}$ & $\begin{array}{l}90.6 \mathrm{t} \\
2.4 \times 2.4 \mathrm{~m}^{2} \\
24.3 \mu \mathrm{sr} \\
1.38 \mathrm{~g} / \mathrm{cm}^{3}\end{array}$ \\
\hline $\begin{array}{l}\text { Number of protons on target } \\
\text { (dead-time corrected) }\end{array}$ & $\begin{array}{l}\text { Density } \varrho=1 \\
\text { Density } \varrho=1 / 3\end{array}$ & $\begin{array}{l}(13.26 \pm 0.13) \times 10^{17} \\
(6.35 \pm 0.07) \times 10^{17}\end{array}$ \\
\hline
\end{tabular}

Table 2

Leakage correction

( $\alpha$ is the fraction of the conventional neutrino rate at $\varrho=1$ which appears to be prompt)

\begin{tabular}{|c|c|c|c|c|c|}
\hline $\begin{array}{c}\mathrm{E}_{y} \\
(\mathrm{GeV})\end{array}$ & $\begin{array}{c}\alpha \\
(\%)\end{array}$ & $\begin{array}{c}\mathrm{E}_{\nu} \\
(\mathrm{GeV})\end{array}$ & $\begin{array}{c}\alpha \\
(\%)\end{array}$ & $\begin{array}{c}\mathrm{E}_{\nu} \\
(\mathrm{GeV})\end{array}$ & $\begin{array}{c}\alpha \\
(\%)\end{array}$ \\
\hline $2-10$ & $10.1 \pm 1.0$ & $40-50$ & $3.3 \pm 1.0$ & $>2$ & $5.5 \pm 0.5$ \\
$10-20$ & $4.6 \pm 1.0$ & $50-60$ & $3.0 \pm 1.0$ & $>20$ & $3.5 \pm 0.4$ \\
$20-30$ & $4.2 \pm 1.0$ & $60-70$ & $2.8 \pm 0.8$ & $>80$ & $2.8 \pm 0.3$ \\
$30-40$ & $3.7 \pm 1.0$ & $70-80$ & $2.8 \pm 0.8$ & & \\
\hline
\end{tabular}


Table 3

Number of protons on target (POT)

\begin{tabular}{|l|c|}
\cline { 2 - 2 } \multicolumn{1}{c|}{} & POT $/ 10^{17}$ \\
\hline Total with detector on & 27.36 \\
\hline - Technical failures $(3.1 \%)$ & 0.85 \\
- Beam information missing $(1.7 \%)$ & 0.47 \\
- Equipment in the beam $(4.2 \%)$ & 1.15 \\
- Measured beam-scraping too high $(5.2 \%)$ & 1.42 \\
- Average electronic dead-time $(16.4 \%)$ & 3.85 \\
\hline Total effective POT for analysis & 19.61 \\
\hline
\end{tabular}

Table 4

Comparison of different live-time measurements

\begin{tabular}{|l|c|c|c|}
\hline \multicolumn{1}{|c|}{ Live-time } & $\begin{array}{c}\text { Scintillator } \\
\text { method }\end{array}$ & $\begin{array}{c}\text { Poisson } \\
\text { method }\end{array}$ & $\begin{array}{c}\text { Clock } \\
\text { method }\end{array}$ \\
\hline Measured & $0.768 \pm 0.006$ & $0.764 \pm 0.008$ & $0.784 \pm 0.005$ \\
Monte Carlo & $0.774 \pm 0.006$ & $0.764 \pm 0.008$ & $0.783 \pm 0.005$ \\
\hline
\end{tabular}

Table 5

Corrections for events with $\mathrm{E}_{\mathrm{sh}} \geq 2 \mathrm{GeV}$

\begin{tabular}{|c|c|c|c|c|}
\hline & \multicolumn{2}{|c|}{$\varrho=1$} & \multicolumn{2}{|c|}{$\varrho=1 / 3$} \\
\hline & $\mathrm{N}(0 \mu)$ & $\mathrm{N}(1 \mu)$ & $\mathrm{N}(0 \mu)$ & $\mathrm{N}(1 \mu)$ \\
\hline Raw data & 1622 & 2202 & 1542 & 2533 \\
\hline $\begin{array}{l}\text { Corrections: } \\
\text { Upstream material } \\
\text { Cosmic-ray background } \\
\pi, \mathrm{K} \rightarrow \mu \text { decay } \\
\text { Soft, side, and hidden } \mu\end{array}$ & $\begin{array}{rr}-3.9 \pm 0.3 \\
-14.1 \pm 2.3 \\
+33.9 \pm 9.1 \\
-146.6 \pm 11.0\end{array}$ & $\begin{array}{rr}-9.8 \pm 0.8 \\
-4.0 \pm 1.8 \\
-33.9 \pm 9.1 \\
+146.6 \pm 11.0\end{array}$ & $\begin{array}{rr}-1.9 \pm 0.2 \\
-7.5 \pm 1.4 \\
+31.5 \pm 8.5 \\
-207.6 \pm 12.4\end{array}$ & $\begin{aligned}-4.9 & \pm 0.4 \\
-2.2 & \pm 0.9 \\
-31.5 & \pm 8.55 \\
+207.6 & \pm 12.4\end{aligned}$ \\
\hline $\begin{array}{l}\text { Corrected data } \\
\pm(\text { stat. error }) \pm \text { (syst. error })\end{array}$ & $\begin{aligned} & 1491.3 \\
\pm & 40.3 \pm 22.4\end{aligned}$ & $\begin{aligned} & 2300.9 \\
\pm & 46.9 \pm 27.3\end{aligned}$ & $\begin{aligned} & 1356.5 \\
\pm & 39.3 \pm 21.5\end{aligned}$ & $\begin{aligned} & 2701.9 \\
\pm & 50.3 \pm 29.4\end{aligned}$ \\
\hline
\end{tabular}




\section{Table 6}

Corrected event rates per tonne and $10^{18}$ POT \pm (stat. error) \pm (syst. error)

\begin{tabular}{|c|c|c|c|}
\hline & \multicolumn{3}{|c|}{ Rates with a cut in shower energy } \\
\hline & $E_{s h} \geq 2 \mathrm{GeV}$ & $\mathrm{E}_{\mathrm{sh}} \geq 20 \mathrm{GeV}$ & $\mathrm{E}_{\mathrm{sh}} \geq 80 \mathrm{GeV}$ \\
\hline $\mathrm{R}(1 \mu): \varrho=1$ & $19.14 \pm 0.39 \pm 0.27$ & $7.68 \pm 0.25 \pm 0.12$ & $1.17 \pm 0.10 \pm 0.02$ \\
\hline$e=1 / 3$ & $46.96 \pm 0.87 \pm 0.68$ & $18.18 \pm 0.55 \pm 0.26$ & $2.47 \pm 0.20 \pm 0.03$ \\
\hline \multirow{4}{*}{$\begin{aligned} \mathrm{R}(0 \mu): & \varrho \\
\varrho & =1 / 3\end{aligned}$} & $12.41 \pm 0.33 \pm 0.21$ & $7.08 \pm 0.25 \pm 0.11$ & $1.89 \pm 0.13 \pm 0.02$ \\
\hline & $23.57 \pm 0.68 \pm 0.49$ & $12.58 \pm 0.48 \pm 0.21$ & $2.89 \pm 0.23 \pm 0.04$ \\
\hline & \multicolumn{3}{|c|}{ Rates with a cut in neutrino energy } \\
\hline & $\mathrm{E}_{\nu} \geq 2 \mathrm{GeV}$ & $\mathrm{E}_{\nu} \geq 20 \mathrm{GeV}$ & $\mathrm{E}_{y} \geq 80 \mathrm{GeV}$ \\
\hline \multirow{4}{*}{$\begin{aligned} \mathrm{R}\left(1 \mu^{-}\right): & \varrho=1 \\
& \varrho=1 / 3 \\
& \text { prompt, extrapolated } \\
& \text { prompt, subtracted }\end{aligned}$} & $18.93 \pm 0.51 \pm 0.53$ & $12.60 \pm 0.38 \pm 0.36$ & $3.17 \pm 0.20 \pm 0.09$ \\
\hline & $49.87 \pm 1.76 \pm 1.34$ & $30.67 \pm 0.85 \pm 0.85$ & $6.90 \pm 0.39 \pm 0.19$ \\
\hline & $2.61 \pm 1.22 \pm 0.44$ & $3.25 \pm 0.72 \pm 0.29$ & $1.26 \pm 0.36 \pm 0.07$ \\
\hline & $3.38 \pm 0.51 \pm 1.60$ & $3.68 \pm 0.38 \pm 0.96$ & $1.12 \pm 0.20 \pm 0.30$ \\
\hline \multirow{4}{*}{$\begin{aligned} \mathrm{R}\left(1 \mu^{+}\right): & \varrho=1 \\
& \varrho=1 / 3 \\
& \text { prompt, extrapolated } \\
& \text { prompt, subtracted }\end{aligned}$} & $6.60 \pm 0.32 \pm 0.18$ & $3.69 \pm 0.21 \pm 0.11$ & $0.68 \pm 0.09 \pm 0.02$ \\
\hline & $16.24 \pm 0.70 \pm 0.43$ & $7.76 \pm 0.41 \pm 0.21$ & $1.19 \pm 0.16 \pm 0.03$ \\
\hline & $1.51 \pm 0.62 \pm 0.16$ & $1.59 \pm 0.38 \pm 0.09$ & $0.42 \pm 0.15 \pm 0.02$ \\
\hline & $2.61 \pm 0.32 \pm 0.47$ & $1.78 \pm 0.21 \pm 0.27$ & $0.47 \pm 0.09 \pm 0.07$ \\
\hline$R(1 \mu): \varrho=1$ & $25.53 \pm 0.61 \pm 0.71$ & $16.29 \pm 0.43 \pm 0.47$ & $3.86 \pm 0.21 \pm 0.11$ \\
\hline \multirow{3}{*}{$\begin{aligned} \mathrm{R}(1 \mu): & \varrho=1 / 3 \\
& \text { prompt, extrapolated } \\
& \text { prompt, subtracted }\end{aligned}$} & $66.11 \pm 1.90 \pm 1.77$ & $38.44 \pm 0.95 \pm 1.06$ & $8.09 \pm 0.42 \pm 0.22$ \\
\hline & $4.12 \pm 1.37 \pm 0.60$ & $4.83 \pm 0.82 \pm 0.38$ & $1.68 \pm 0.39 \pm 0.09$ \\
\hline & $5.99 \pm 0.61 \pm 1.87$ & $5.47 \pm 0.43 \pm 1.05$ & $1.60 \pm 0.21 \pm 0.32$ \\
\hline
\end{tabular}


Table 7

Corrected event rates per tonne and $10^{20} \mathrm{POT}$

\begin{tabular}{|c|r|r|r|r|}
\cline { 2 - 5 } \multicolumn{1}{c|}{} & \multicolumn{2}{c|}{$\varrho=1$} & \multicolumn{2}{c|}{$\varrho=1 / 3$} \\
\hline $\mathrm{E}_{\mathrm{sh}}$ & $\mathrm{R}(1 \mu)$ & $\mathrm{R}(0 \mu)$ & $\mathrm{R}(1 \mu)$ & $\mathrm{R}(0 \mu)$ \\
$(\mathrm{GeV})$ & & & & \\
\hline $2-10$ & $681.3 \pm 23.0$ & $295.1 \pm 17.2$ & $1741.3 \pm 52.4$ & $660.7 \pm 38.0$ \\
$10-20$ & $464.9 \pm 19.2$ & $237.9 \pm 14.8$ & $1136.8 \pm 42.8$ & $438.3 \pm 30.2$ \\
$20-30$ & $232.6 \pm 13.8$ & $151.1 \pm 11.5$ & $610.8 \pm 31.9$ & $311.0 \pm 24.1$ \\
$30-40$ & $146.9 \pm 10.9$ & $107.1 \pm 9.7$ & $350.7 \pm 24.2$ & $205.1 \pm 19.4$ \\
$40-50$ & $101.1 \pm 9.0$ & $77.3 \pm 8.2$ & $258.1 \pm 20.8$ & $143.2 \pm 16.3$ \\
$50-60$ & $71.3 \pm 7.6$ & $74.3 \pm 7.9$ & $159.9 \pm 16.4$ & $122.6 \pm 14.9$ \\
$60-70$ & $55.5 \pm 6.7$ & $64.0 \pm 7.3$ & $116.4 \pm 14.0$ & $112.9 \pm 14.2$ \\
$70-80$ & $43.2 \pm 5.9$ & $44.8 \pm 6.1$ & $75.6 \pm 11.3$ & $73.8 \pm 11.5$ \\
$80-90$ & $37.1 \pm 5.5$ & $38.5 \pm 5.7$ & $59.6 \pm 10.1$ & $55.1 \pm 9.8$ \\
$90-100$ & $18.9 \pm 3.9$ & $42.5 \pm 6.0$ & $45.3 \pm 8.9$ & $55.3 \pm 9.8$ \\
$100-120$ & $27.3 \pm 4.8$ & $53.1 \pm 6.6$ & $73.1 \pm 11.1$ & $84.8 \pm 12.3$ \\
$120-140$ & $18.7 \pm 3.8$ & $24.3 \pm 4.6$ & $28.4 \pm 6.9$ & $46.1 \pm 9.0$ \\
$140-160$ & $9.4 \pm 2.8$ & $18.7 \pm 4.0$ & $19.6 \pm 5.8$ & $25.3 \pm 6.7$ \\
$160-180$ & $3.2 \pm 1.6$ & $8.2 \pm 2.6$ & $15.7 \pm 5.2$ & $14.0 \pm 5.0$ \\
$180-200$ & $2.8 \pm 1.4$ & $4.0 \pm 1.8$ & $5.2 \pm 2.9$ & $8.8 \pm 4.0$ \\
\hline 2 & $1914.3 \pm 39.0$ & $1240.9 \pm 33.5$ & $4696.5 \pm 87.4$ & $2357.0 \pm 68.2$ \\
20 & $768.1 \pm 25.0$ & $708.0 \pm 24.7$ & $1818.4 \pm 55.2$ & $1258.0 \pm 47.9$ \\
80 & $117.5 \pm 9.8$ & $189.4 \pm 12.7$ & $246.9 \pm 20.5$ & $289.4 \pm 22.6$ \\
\hline
\end{tabular}

Table 8

Comparison of two methods of analysis for $(1 \mu)$ events with $\mathrm{E}_{\nu}>20 \mathrm{GeV}$

\begin{tabular}{|l|c|c|c|}
\hline \multicolumn{1}{|c|}{ Method } & Momentum cut & $\varrho=1$ & $\varrho=1 / 3$ \\
\hline Unfolding & $\mathrm{p}_{\mu}>1 \mathrm{GeV} / \mathrm{c}$ & $16.27 \pm 0.43$ & $37.55 \pm 0.92$ \\
Acceptance & $\mathrm{p}_{\mu}>5 \mathrm{GeV} / \mathrm{c}$ & $16.29 \pm 0.43$ & $38.43 \pm 0.94$ \\
\hline
\end{tabular}




\section{Table 9}

Rates of charged-current $\nu_{\mu}$ and $\bar{\nu}_{\mu}$ interactions per tonne and $10^{20}$ POT

\begin{tabular}{|c|c|c|c|c|}
\hline \multirow[b]{2}{*}{$\begin{array}{c}\mathrm{E}_{\nu} \\
(\mathrm{GeV})\end{array}$} & \multicolumn{2}{|c|}{$\varrho=1$} & \multicolumn{2}{|c|}{$\varrho=1 / 3$} \\
\hline & $\mathrm{R}\left(\nu_{\mu}\right)$ & $\mathbf{R}\left(\bar{\nu}_{\mu}\right)$ & $\mathrm{R}\left(\nu_{\mu}\right)$ & $\mathrm{R}\left(\bar{\nu}_{\mu}\right)$ \\
\hline $2-10$ & $317.4 \pm 27.3$ & $168.7 \pm 22.0$ & $953.8 \pm 130.0$ & $470.0 \pm 47.0$ \\
\hline $10-20$ & $315.5 \pm 21.7$ & $121.7 \pm 11.7$ & $966.0 \pm 83.6$ & $378.0 \pm 32.0$ \\
\hline $20-30$ & $283.0 \pm 18.2$ & $89.9 \pm 11.5$ & $708.2 \pm 42.7$ & $226.0 \pm 22.5$ \\
\hline $30-40$ & $189.5 \pm 14.8$ & $68.7 \pm 8.1$ & $537.8 \pm 36.8$ & $151.4 \pm 16.8$ \\
\hline $40-50$ & $168.1 \pm 13.6$ & $56.7 \pm 7.7$ & $373.8 \pm 28.3$ & $110.0 \pm 18.3$ \\
\hline $50-60$ & $114.9 \pm 10.5$ & $44.6 \pm 6.7$ & $289.7 \pm 24.4$ & $67.9 \pm 11.7$ \\
\hline $60-70$ & $99.7 \pm 10.6$ & $23.3 \pm 5.0$ & $268.1 \pm 25.6$ & $55.2 \pm 10.2$ \\
\hline $70-80$ & $87.5 \pm 9.4$ & $17.7 \pm 4.4$ & $200.0 \pm 22.4$ & $46.8 \pm 9.4$ \\
\hline $80-90$ & $68.1 \pm 7.8$ & $17.0 \pm 3.8$ & $160.2 \pm 18.3$ & $38.5 \pm 9.5$ \\
\hline $90-100$ & $63.6 \pm 8.1$ & $15.5 \pm 3.9$ & $130.0 \pm 18.9$ & $31.7 \pm 8.6$ \\
\hline $100-120$ & $77.1 \pm 9.7$ & $14.5 \pm 5.2$ & $158.9 \pm 18.1$ & $31.6 \pm 8.6$ \\
\hline $120-140$ & $41.3 \pm 7.4$ & $9.2 \pm 3.0$ & $110.9 \pm 15.6$ & $9.6 \pm 3.0$ \\
\hline $140-160$ & $30.8 \pm 6.8$ & $7.8 \pm 2.6$ & $52.4 \pm 11.6$ & $6.4 \pm 2.3$ \\
\hline $160-180$ & $24.9 \pm 7.0$ & $3.2 \pm 1.6$ & $45.6 \pm 10.6$ & $0.8 \pm 0.6$ \\
\hline $180-200$ & $11.7 \pm 3.2$ & $1.0 \pm 0.4$ & $31.8 \pm 4.21$ & $0.2 \pm 0.1$ \\
\hline 2 & $1893.0 \pm 51.5$ & $659.7 \pm 32.3$ & $4987.2 \pm 176.4$ & $1624.1 \pm 70.3$ \\
\hline 20 & $1260.1 \pm 37.8$ & $369.2 \pm 20.6$ & $3067.4 \pm 85.2$ & $776.2 \pm 41.2$ \\
\hline 80 & $317.5 \pm 19.6$ & $68.4 \pm 8.7$ & $689.7 \pm 39.1$ & $118.9 \pm 15.9$ \\
\hline
\end{tabular}


Table 10

Charged-current $\left(\nu_{\mathrm{e}}+\bar{\nu}_{\mathrm{e}}\right)$ events per tonne and $10^{20}$ POT

\begin{tabular}{|c|c|c|c|c|}
\cline { 2 - 5 } \multicolumn{1}{c|}{} & \multicolumn{2}{c|}{ Direct search } & \multicolumn{2}{c|}{ Extrapolation of $\mathrm{R}(0 \mu)-\langle\mathrm{R}\rangle \times \mathrm{R}(1 \mu)$} \\
\hline $\mathrm{E}_{\nu}$ & $\varrho=1$ & $\varrho=1 / 3$ & $\mathrm{E}_{\nu}$ & Prompt (1e) \\
$(\mathrm{GeV})$ & & & & \\
\hline $2-10$ & $38.0 \pm 19.5$ & $48.7 \pm 43.1$ & $2-10$ & $6.1 \pm 3.7$ \\
$10-20$ & $26.0 \pm 16.4$ & $72.8 \pm 27.4$ & $10-20$ & $44.9 \pm 14.2$ \\
$20-30$ & $36.8 \pm 9.9$ & $65.9 \pm 21.1$ & $20-30$ & $46.3 \pm 19.3$ \\
$30-40$ & $38.0 \pm 8.4$ & $54.7 \pm 14.8$ & $30-40$ & $37.4 \pm 16.8$ \\
$40-50$ & $55.1 \pm 8.5$ & $71.6 \pm 14.5$ & $40-50$ & $31.2 \pm 13.7$ \\
$50-60$ & $43.6 \pm 10.6$ & $79.9 \pm 18.8$ & $50-60$ & $37.3 \pm 13.7$ \\
$60-70$ & $30.5 \pm 7.8$ & $47.1 \pm 16.8$ & $60-70$ & $25.8 \pm 11.6$ \\
$70-80$ & $42.1 \pm 11.7$ & $23.6 \pm 24.5$ & $70-80$ & $17.8 \pm 9.8$ \\
$80-90$ & $49.3 \pm 11.2$ & $41.8 \pm 16.6$ & $80-90$ & $18.3 \pm 8.9$ \\
$90-100$ & $27.1 \pm 8.8$ & $46.2 \pm 16.8$ & $90-100$ & $28.4 \pm 8.9$ \\
$100-120$ & $40.7 \pm 9.0$ & $37.1 \pm 15.7$ & $100-120$ & $29.3 \pm 10.1$ \\
120 & $14.3 \pm 12.7$ & $4.9 \pm 18.2$ & $120-140$ & $6.8 \pm 6.9$ \\
& & & $140-160$ & $9.3 \pm 4.8$ \\
& & & $160-180$ & $4.9 \pm 3.8$ \\
& & & $180-200$ & $0.5 \pm 1.7$ \\
\hline 20 & $441.5 \pm 40.6$ & $594.1 \pm 76.4$ & 2 & $344.3 \pm 42.7$ \\
20 & $377.5 \pm 31.6$ & $472.7 \pm 57.0$ & 20 & $293.8 \pm 40.2$ \\
\hline & $131.4 \pm 21.1$ & $130.0 \pm 33.7$ & 80 & $97.7 \pm 18.9$ \\
\hline & & & & \\
\hline & & & & \\
\hline
\end{tabular}


Table 11

Rates of charged-current events from conventional neutrino sources for $\varrho=1$ from muon flux measurements and cascade calculations per tonne and $10^{20} \mathrm{POT}$

\begin{tabular}{|c|c|c|c|c|}
\cline { 2 - 5 } \multicolumn{1}{c|}{} & \multicolumn{2}{c|}{ Muon neutrinos } & \multicolumn{2}{c|}{ Electron-neutrinos } \\
\hline $\mathrm{E}_{\nu}$ & $\mathrm{R}\left(\nu_{\mu}\right)$ & $\mathrm{R}\left(\bar{\nu}_{\mu}\right)$ & $\mathrm{R}\left(\nu_{\mathrm{e}}\right)$ & $\mathrm{R}\left(\bar{\nu}_{\mathrm{e}}\right)$ \\
\hline $\mathrm{GeV})$ & & & & \\
\hline $2-10$ & 367.0 & 109.0 & 20.7 & 8.4 \\
$10-20$ & 303.5 & 98.5 & 20.0 & 10.5 \\
$20-30$ & 222.3 & 64.0 & 12.0 & 7.8 \\
$30-40$ & 154.3 & 38.6 & 8.5 & 4.8 \\
$40-50$ & 108.6 & 27.5 & 6.7 & 3.1 \\
$50-60$ & 84.3 & 18.3 & 4.6 & 1.9 \\
$60-70$ & 66.0 & 12.8 & 3.2 & 1.22 \\
$70-80$ & 54.8 & 9.2 & 2.4 & 0.75 \\
$80-90$ & 43.6 & 6.6 & 1.77 & 0.48 \\
$90-100$ & 32.7 & 4.4 & 1.28 & 0.35 \\
$100-120$ & 47.8 & 5.4 & 1.70 & 0.40 \\
$120-140$ & 30.2 & 2.5 & 0.76 & 0.20 \\
$140-160$ & 20.6 & 1.1 & 0.36 & 0.10 \\
$160-180$ & 11.4 & 0.54 & 0.19 & 0.03 \\
$180-200$ & 7.6 & 0.23 & 0.10 & 0.02 \\
\hline 2 & $1554.7 \pm 152.9$ & $398.6 \pm 42.6$ & $84.2 \pm 11.4$ & $40.0 \pm 6.2$ \\
20 & $892.3 \pm 89.7$ & $189.8 \pm 24.9$ & $43.6 \pm 5.3$ & $21.1 \pm 3.3$ \\
80 & $205.1 \pm 29.3$ & $20.9 \pm 6.4$ & $6.2 \pm 0.8$ & $1.6 \pm 0.3$ \\
\hline
\end{tabular}

Table 12

Comparison of the event rates for the two experiments performed by this collaboration.

Events per tonne and $10^{18}$ POT for $E_{s h}>2 \mathrm{GeV}$. Only statistical errors are given.

\begin{tabular}{|l|c|c|c|c|}
\cline { 2 - 5 } \multicolumn{1}{c|}{} & \multicolumn{2}{c|}{$\varrho=1$} & \multicolumn{2}{c|}{$\varrho=1 / 3$} \\
\cline { 2 - 5 } \multicolumn{1}{c|}{$\mathrm{R}(1 \mu)$} & $\mathrm{R}(0 \mu)$ & $\mathrm{R}(1 \mu)$ & $\mathrm{R}(0 \mu)$ \\
\hline $\mathrm{BD} 1982$ & $19.14 \pm 0.39$ & $12.41 \pm 0.33$ & $46.96 \pm 0.87$ & $23.57 \pm 0.68$ \\
$\mathrm{BD} 1979^{\mathrm{a})}$ & $18.84 \pm 0.88$ & $12.27 \pm 0.76$ & $44.51 \pm 2.20$ & $23.68 \pm 1.75$ \\
$\mathrm{BD} 1979^{\mathrm{b})}$ & $19.67 \pm 0.92$ & $12.58 \pm 0.78$ & $46.87 \pm 2.32$ & $24.64 \pm 1.82$ \\
\hline
\end{tabular}

a) Constant scaling factor of 3.02 .

b) Scaling factor taking angular distribution into account. 
Table 13

Rates of $\mathrm{R}(0 \mu)-\langle\mathrm{R}\rangle \times \mathrm{R}(1 \mu)$ and of $\mathrm{R}(1 \mathrm{e})$

Events per tonne and $10^{18}$ POT \pm (statistical error) \pm (systematic error)

\begin{tabular}{|l|c|c|c|}
\cline { 2 - 4 } \multicolumn{1}{c|}{} & \multicolumn{3}{c|}{ Event rates for $\mathrm{R}(0 \mu)-\langle\mathrm{R}\rangle \times \mathrm{R}(1 \mu)$} \\
\cline { 2 - 4 } & $\mathrm{E}_{\mathrm{sh}} \geq 2 \mathrm{GeV}$ & $\mathrm{E}_{\mathrm{sh}} \geq 20 \mathrm{GeV}$ & $\mathrm{E}_{\mathrm{sh}} \geq 80 \mathrm{GeV}$ \\
\hline$\varrho=1$ & $6.03 \pm 0.36 \pm 0.23$ & $4.52 \pm 0.26 \pm 0.12$ & $1.50 \pm 0.13 \pm 0.02$ \\
$\varrho=1 / 3$ \\
Prompt, extrapolated & $7.91 \pm 0.74 \pm 0.58$ & $6.52 \pm 0.51 \pm 0.23$ & $2.07 \pm 0.24 \pm 0.03$ \\
& $5.03 \pm 0.67 \pm 0.15$ & $3.48 \pm 0.48 \pm 0.11$ & $1.21 \pm 0.23 \pm 0.03$ \\
\hline & \multicolumn{3}{|c|}{$\mathrm{CC}$ event rates for $\nu_{\mathrm{e}}+\bar{\nu}_{\mathrm{e}}$} \\
\hline & $\mathrm{E}_{\nu} \geq 2 \mathrm{GeV}$ & $\mathrm{E}_{\nu} \geq 20 \mathrm{GeV}$ & $\mathrm{E}_{\nu} \geq 80 \mathrm{GeV}$ \\
\hline Prompt, extrapolated & $3.44 \pm 0.43 \pm 0.12$ & $2.94 \pm 0.40 \pm 0.09$ & $0.98 \pm 0.19 \pm 0.03$ \\
Prompt, subtracted & $3.45 \pm 0.28 \pm 0.12$ & $3.19 \pm 0.22 \pm 0.14$ & $1.13 \pm 0.11 \pm 0.03$ \\
\hline
\end{tabular}

Table 14

Rates of directly detected (1e) events.

Events per tonne and $10^{18}$ POT \pm (statistical error) \pm (systematic error)

\begin{tabular}{|l|c|c|c|}
\cline { 2 - 4 } \multicolumn{1}{c|}{} & $\mathrm{E}_{\nu} \geq 2 \mathrm{GeV}$ & $\mathrm{E}_{\nu} \geq 20 \mathrm{GeV}$ & $\mathrm{E}_{\nu} \geq 80 \mathrm{GeV}$ \\
\hline$\varrho=1$ & $4.41 \pm 0.41 \pm 0.33$ & $3.77 \pm 0.32 \pm 0.28$ & $1.31 \pm 0.21 \pm 0.10$ \\
$\varrho=1 / 3$ & $5.94 \pm 0.76 \pm 0.45$ & $4.72 \pm 0.57 \pm 0.36$ & $1.30 \pm 0.34 \pm 0.10$ \\
Prompt, extrapolated & $3.61 \pm 0.74 \pm 0.28$ & $3.28 \pm 0.56 \pm 0.25$ & $1.32 \pm 0.36 \pm 0.10$ \\
Prompt, subtracted & $3.17 \pm 0.41 \pm 0.38$ & $3.13 \pm 0.32 \pm 0.30$ & $1.24 \pm 0.21 \pm 0.10$ \\
\hline
\end{tabular}




\section{Table 15}

Asymmetries and ratios of prompt electron-neutrino and muon-neutrino fluxes.

The asymmetry is defined as $[R(1 \mu)-R(1 e)] /[R(1 \mu)+R(1 e)]$ and the ratio as $R(1 e) / R(1 \mu)$.

\begin{tabular}{|c|c|c|c|}
\hline & $\mathrm{E}_{\nu} \quad 2 \mathrm{GeV}$ & $20 \mathrm{GeV}$ & $80 \mathrm{GeV}$ \\
\hline \multicolumn{4}{|c|}{ Asymmetries in $\%$ with electron rates from $\mathbf{R}(0 \mu)-\langle\mathbf{R}\rangle \times \mathbf{R}(1 \mu)$} \\
\hline Extrapolation & $9.0 \pm 19.0 \pm 6.9$ & $24.4 \pm 10.2 \pm 3.4$ & $26.6 \pm 14.0 \pm 2.0$ \\
\hline Subtraction ${ }^{a)}$ & $9.6 \pm 16.9 \pm 8.0$ & $21.1 \pm 8.7 \pm 4.3$ & $19.8 \pm 12.0 \pm 2.6$ \\
\hline \multicolumn{4}{|c|}{ Asymmetries in $\%$ with electron rates from direct search } \\
\hline Extrapolation & $6.6 \pm 19.4 \pm 7.6$ & $19.1 \pm 11.6 \pm 4.8$ & $12.1 \pm 17.7 \pm 4.3$ \\
\hline Subtraction $^{\text {a) }}$ & $13.0 \pm 17.5 \pm 9.1$ & $21.4 \pm 9.4 \pm 5.7$ & $15.4 \pm 14.1 \pm 10.4$ \\
\hline \multicolumn{4}{|c|}{ Ratios with electron rates from $\mathbf{R}(0 \mu)-\langle\mathbf{R}\rangle \times \mathbf{R}(1 \mu)$} \\
\hline Extrapolation & $0.83+0.45+0.16$ & $\begin{array}{c}0.61+0.17+0.05 \\
-0.11-0.04\end{array}$ & $\begin{array}{l}0.58+0.25+0.03 \\
-0.13-0.03\end{array}$ \\
\hline Subtraction $^{\text {a) }}$ & $\begin{array}{r}+0.43+0.17 \\
0.83-0.21-0.12\end{array}$ & $\begin{array}{r}0.65+0.15+0.07 \\
-0.10-0.06\end{array}$ & $\begin{array}{r}0.67+0.22+0.04 \\
-0.13-0.03\end{array}$ \\
\hline \multicolumn{4}{|c|}{ Ratios with electron rates from direct search } \\
\hline Extrapolation & $\begin{array}{r}0.88+0.56+0.17 \\
-0.25-0.12\end{array}$ & $\begin{array}{r}0.68+0.22+0.08 \\
-0.13-0.06\end{array}$ & $\begin{array}{r}0.78+0.44+0.07 \\
-0.21-0.06\end{array}$ \\
\hline Subtraction $^{\text {a) }}$ & $\begin{array}{r}0.77+0.43+0.19 \\
-0.20-0.13\end{array}$ & $\begin{array}{r}0.65+0.16+0.09 \\
-0.11-0.07\end{array}$ & $\begin{array}{c}0.73+0.30+0.07 \\
-0.16-0.06\end{array}$ \\
\hline
\end{tabular}

a) Subtraction method used only for prompt $\nu_{\mathrm{e}}$. 


\section{Figure captions}

Fig. 1 General view of the experimental set-up.

Fig. 2 Target arrangement.

Fig. 3 Display of a charged-current and a neutral-current event. Scintillation counter and proportional drift-tube hits are shown as lines and dots, respectively.

Fig. 4 Muon flux in the pits of the iron shield for different target arrangements.

Fig. 5 Extrapolation of the muon fluxes measured in four different pits to infinite density. The fluxes are normalized to the rates measured for $\varrho=1 / 3$.

Fig. 6 a) Proton loss rate in arbitrary units (the arrow indicates the threshold for rejection). b) Ratio of two BCT measurements. c) Ratio of a solid-state detector and a BCT measurement.

Fig. 7 Event rate (arbitrary units) as a function of the run number for two different densities. The horizontal lines show the average rates; the vertical lines indicate the subdivision of data into three different running periods.

Fig. 8 Distribution of the deviation of event rates of individual runs from the mean rate.

Fig. 9 Efficiency for a) muon finding, b) muon charge measurement.

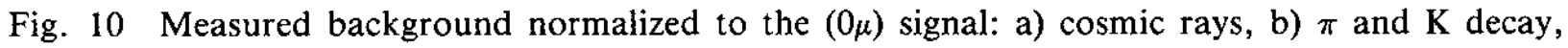
c) soft, side, and hidden muons.

Fig. 11 Energy distributions of charged-current $\nu_{\mu}$ and $\bar{\nu}_{\mu}$ events, after unfolding of the experimental resolutions.

Fig. 12 Distribution of the inelasticity $y=E_{h} / E_{\nu}$ of $(1 \mu)$ events recorded with the $\varrho=1$ target. The curve shows the prediction for charged-current $\nu_{\mu}$ and $\bar{\nu}_{\mu}$ events.

Fig. 13 Result of the electron shower search algorithm applied to $15 \mathrm{GeV}$ test-beam events.

Fig. 14 Analysis of a y-distribution in the search for electromagnetic showers: a) measured y-distribution (full line) and background component (dashed line); b) y-distribution after background subtraction (full line) and Monte Carlo prediction (dashed line).

Fig. 15 Spectrum of conventional charged-current $\left(\nu_{\mu}+\bar{\nu}_{\mu}\right)$ events for $\varrho=1$ from extrapolation (data points) and from muon flux measurement and cascade simulation (histogram).

Fig. 16 Energy spectrum of prompt charged-current $\left(\nu_{\mu}+\bar{\nu}_{\mu}\right)$ events. The curve shows the prediction of the charm production model. 
Fig. 17 Energy spectrum of prompt $(0 \mu)$ events not induced by $\nu_{\mu}$ or $\bar{\nu}_{\mu}$ interactions. The curve shows the prediction of the charm production model.

Fig. 18 Energy spectrum of prompt charged-current $\left(\nu_{\mathrm{e}}+\bar{\nu}_{\mathrm{e}}\right)$ interactions from unfolding of the prompt $(0 \mu)$ spectrum. The curve shows the prediction of the charm production model.

Fig. 19 Energy spectrum of prompt (1e) events obtained by subtraction of the conventional background from directly determined electrons. The curve shows the prediction of the charm production model.

Fig. 20 Asymmetry of prompt $\bar{\nu}_{\mu}$ and $\nu_{\mu}$ fluxes.

Fig. 21 Asymmetry of prompt $\left(\nu_{\mathrm{e}}+\bar{\nu}_{\mathrm{e}}\right)$ and $\left(\nu_{\mu}+\bar{\nu}_{\mu}\right)$ fluxes. 


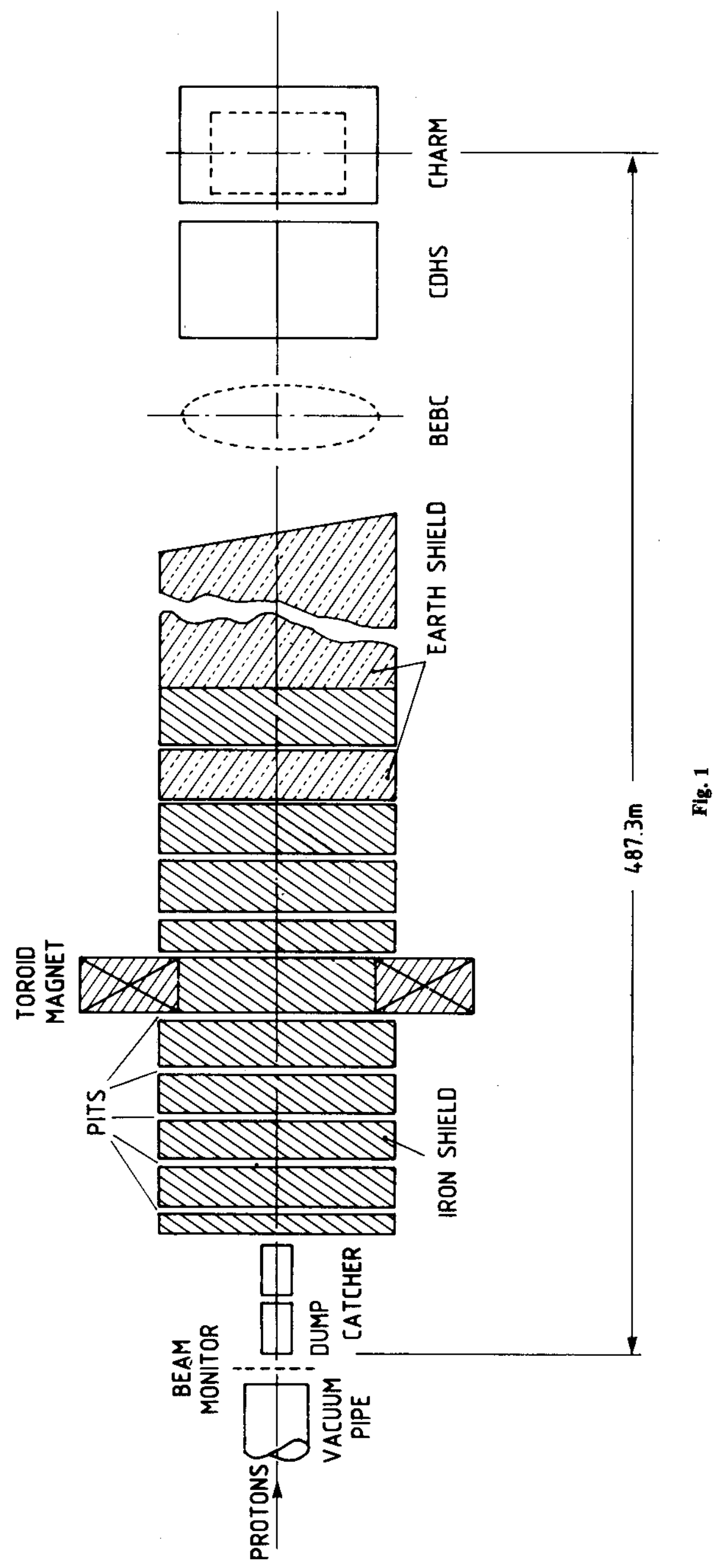




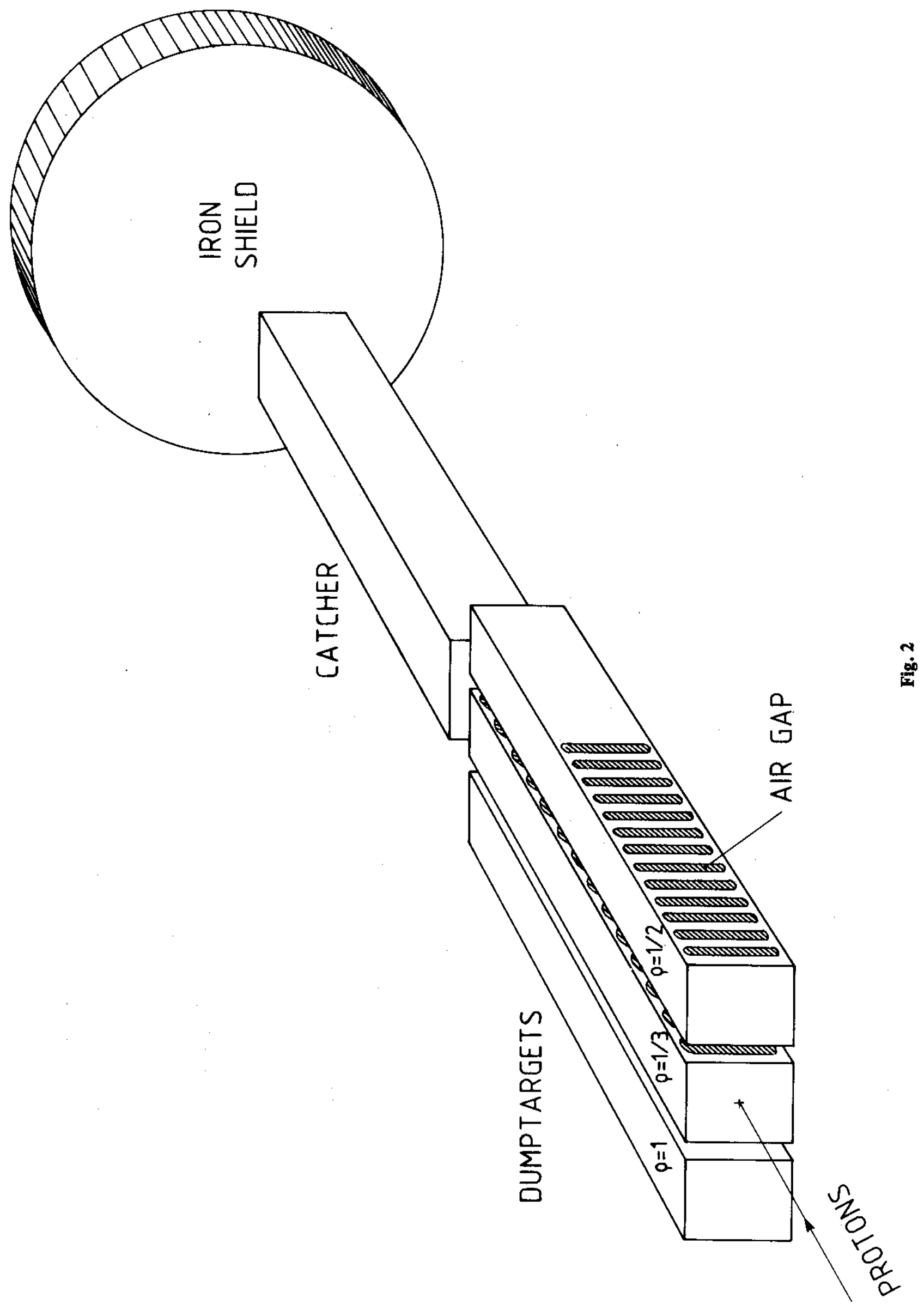



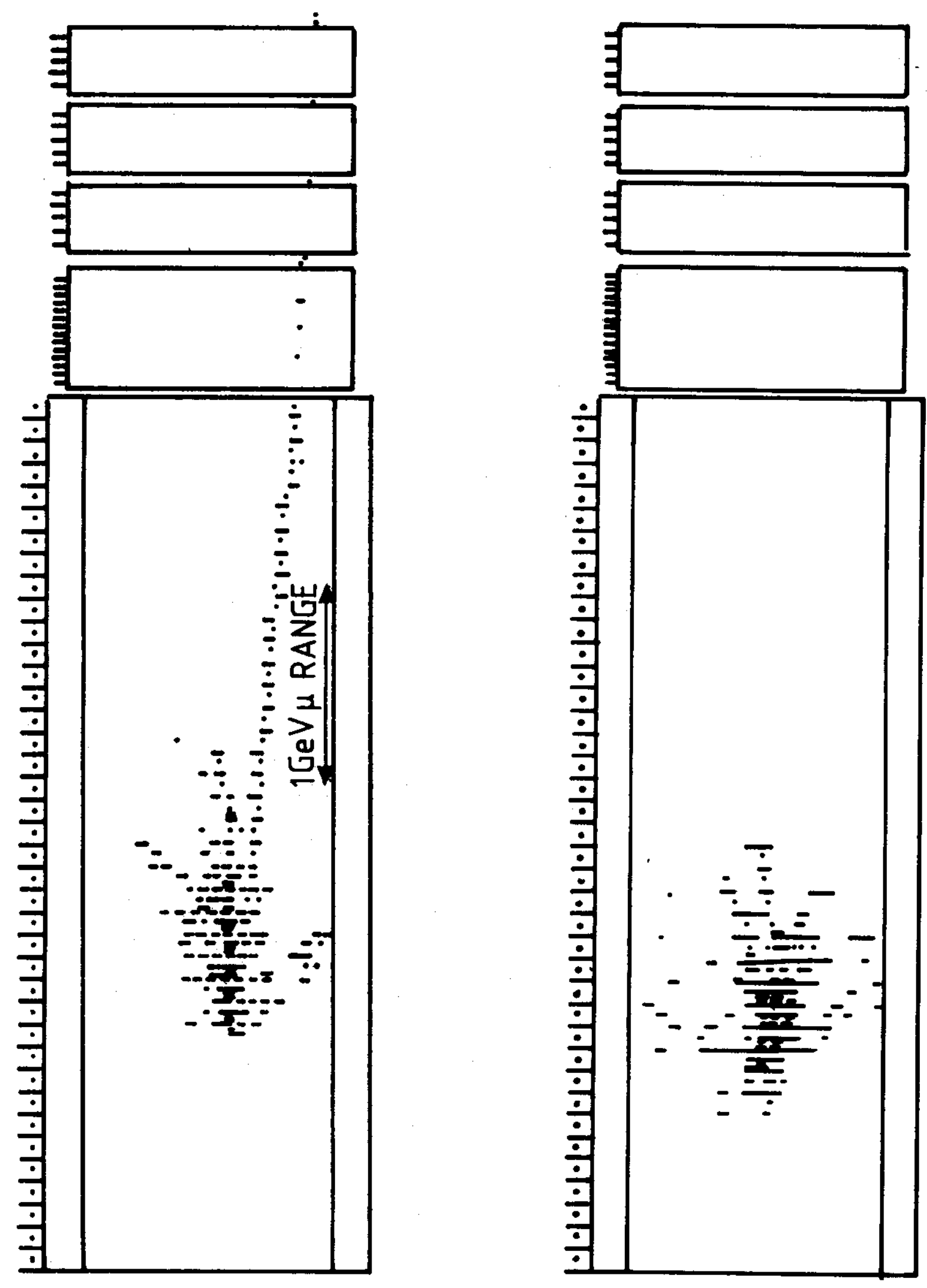

$m$ 


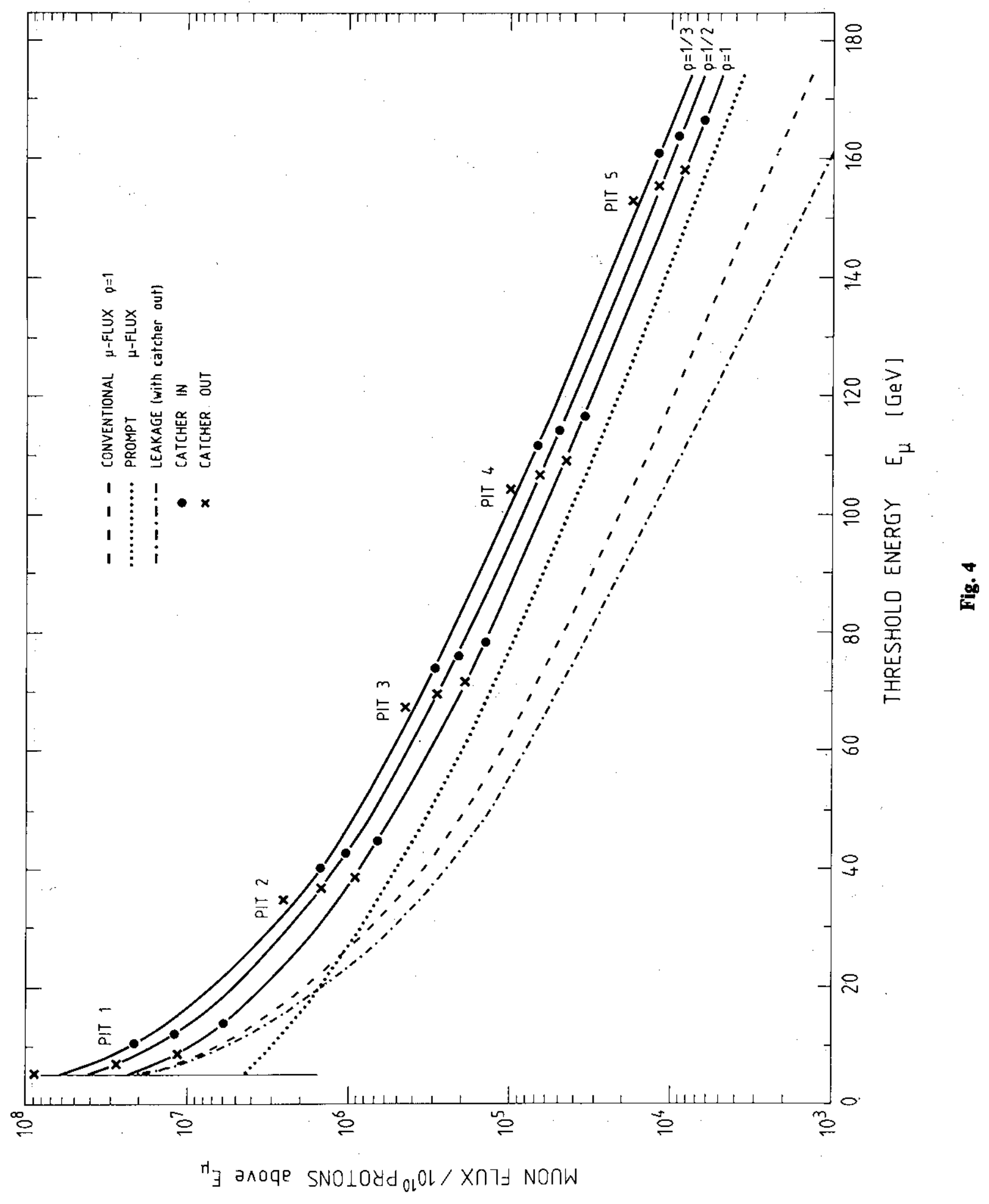




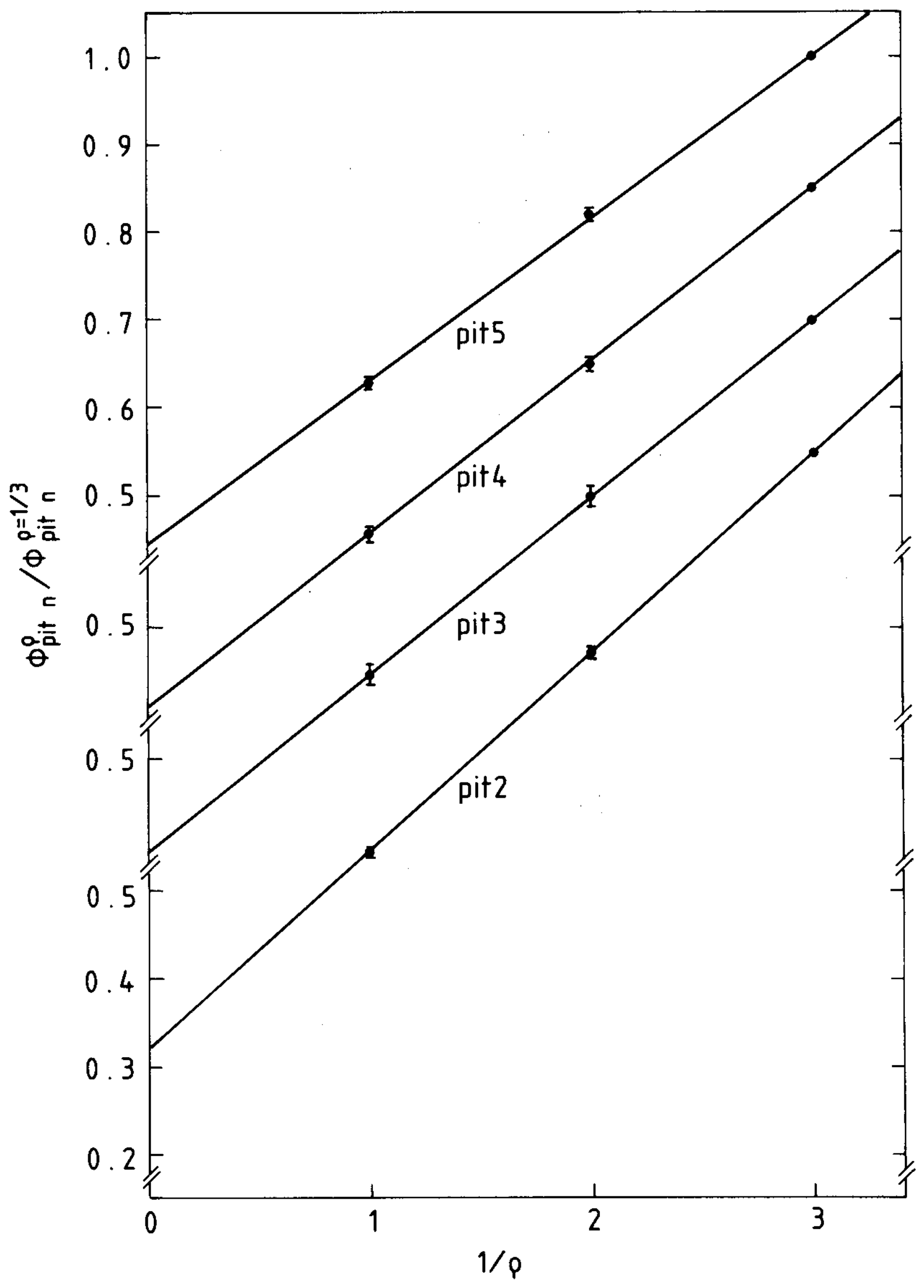

Fig. 5 


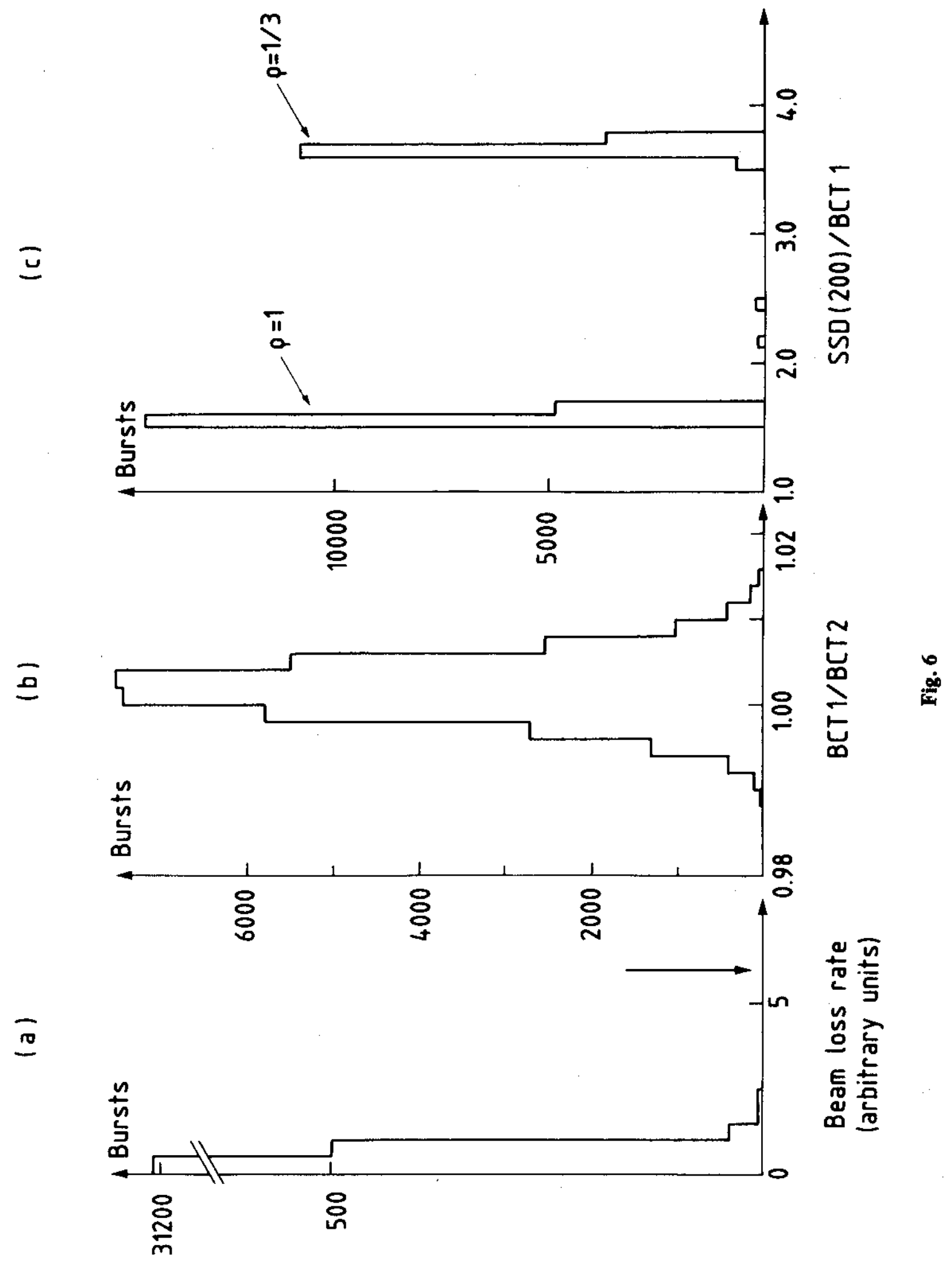




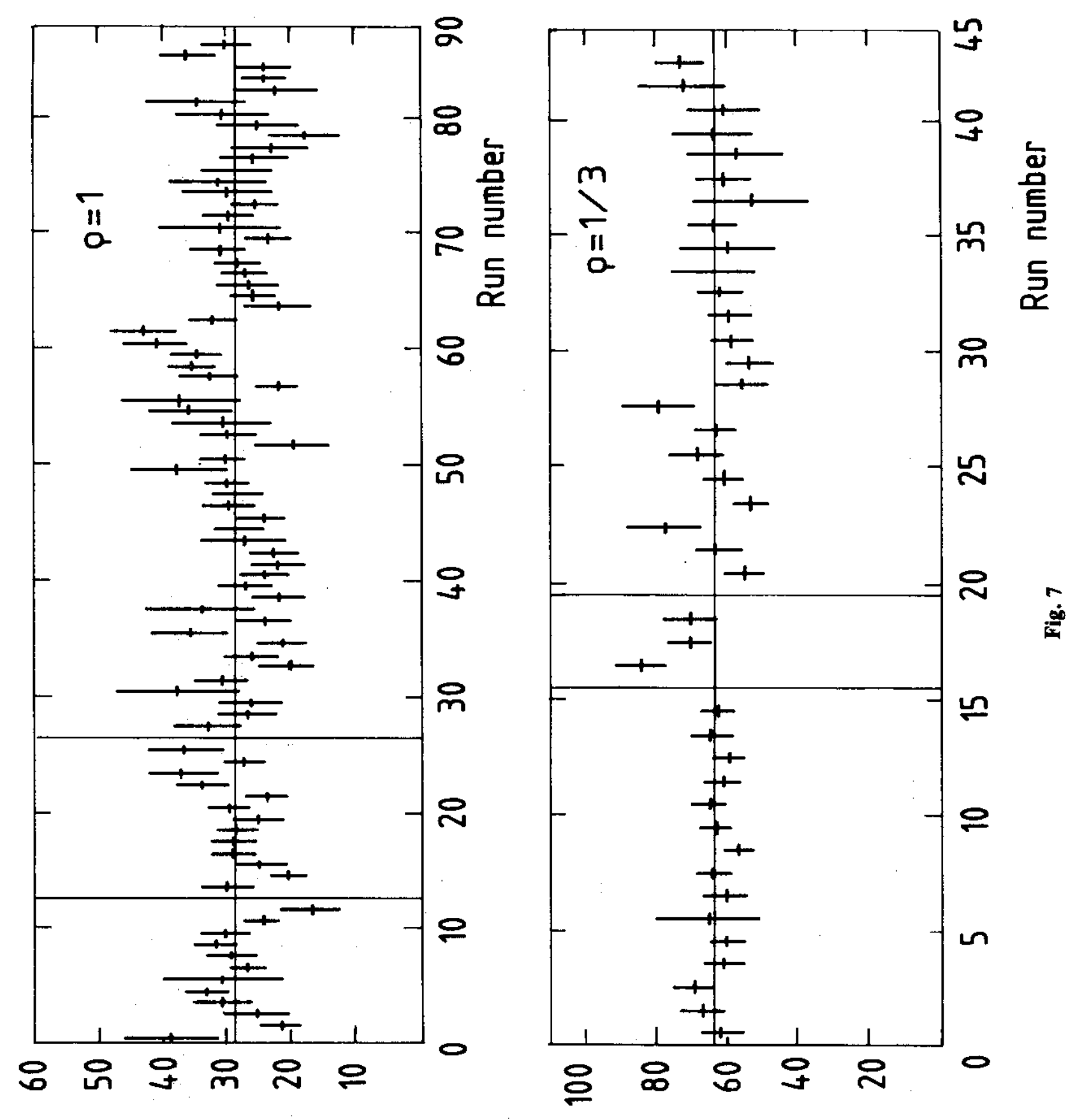

10d/sfuan] 


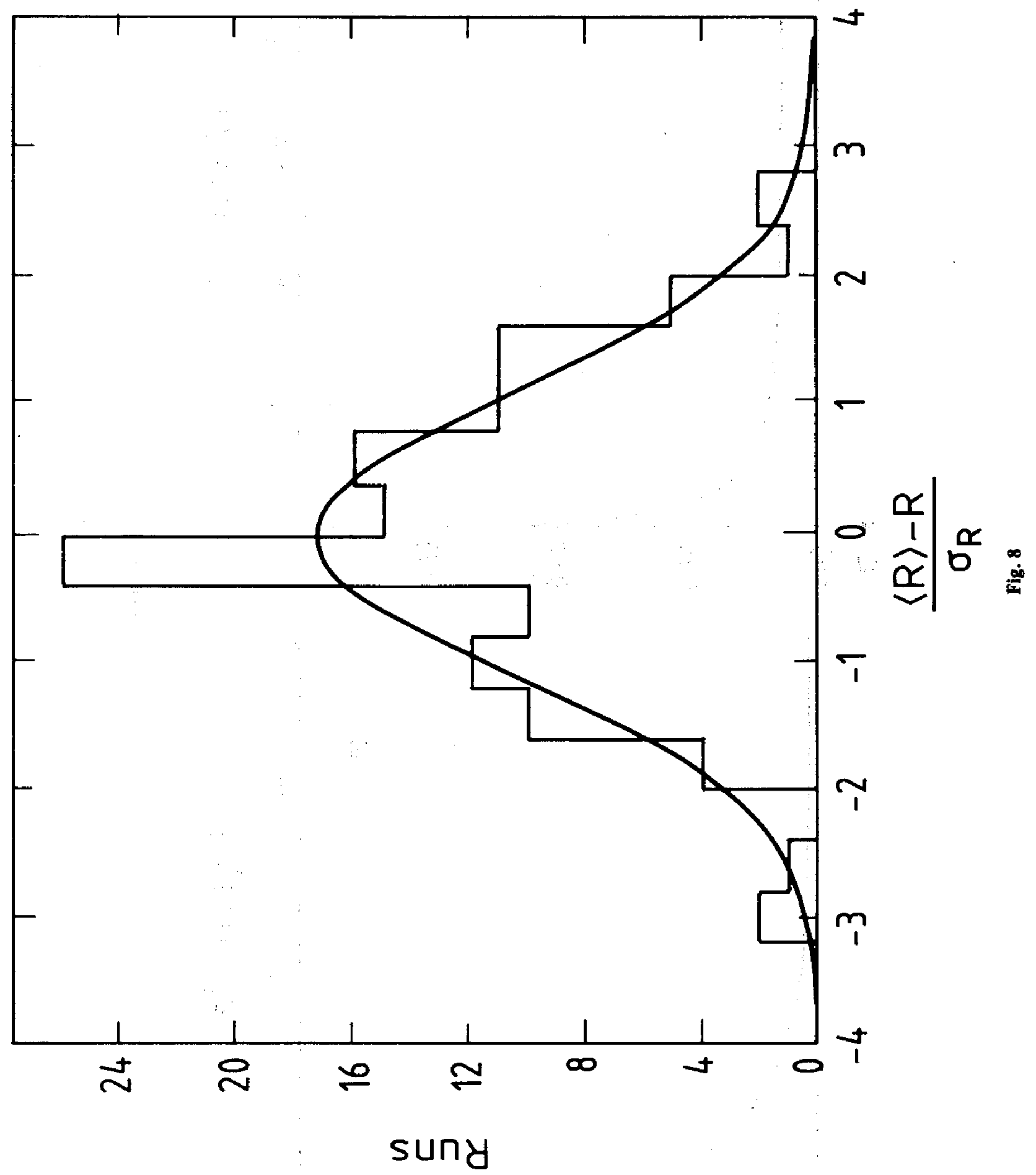



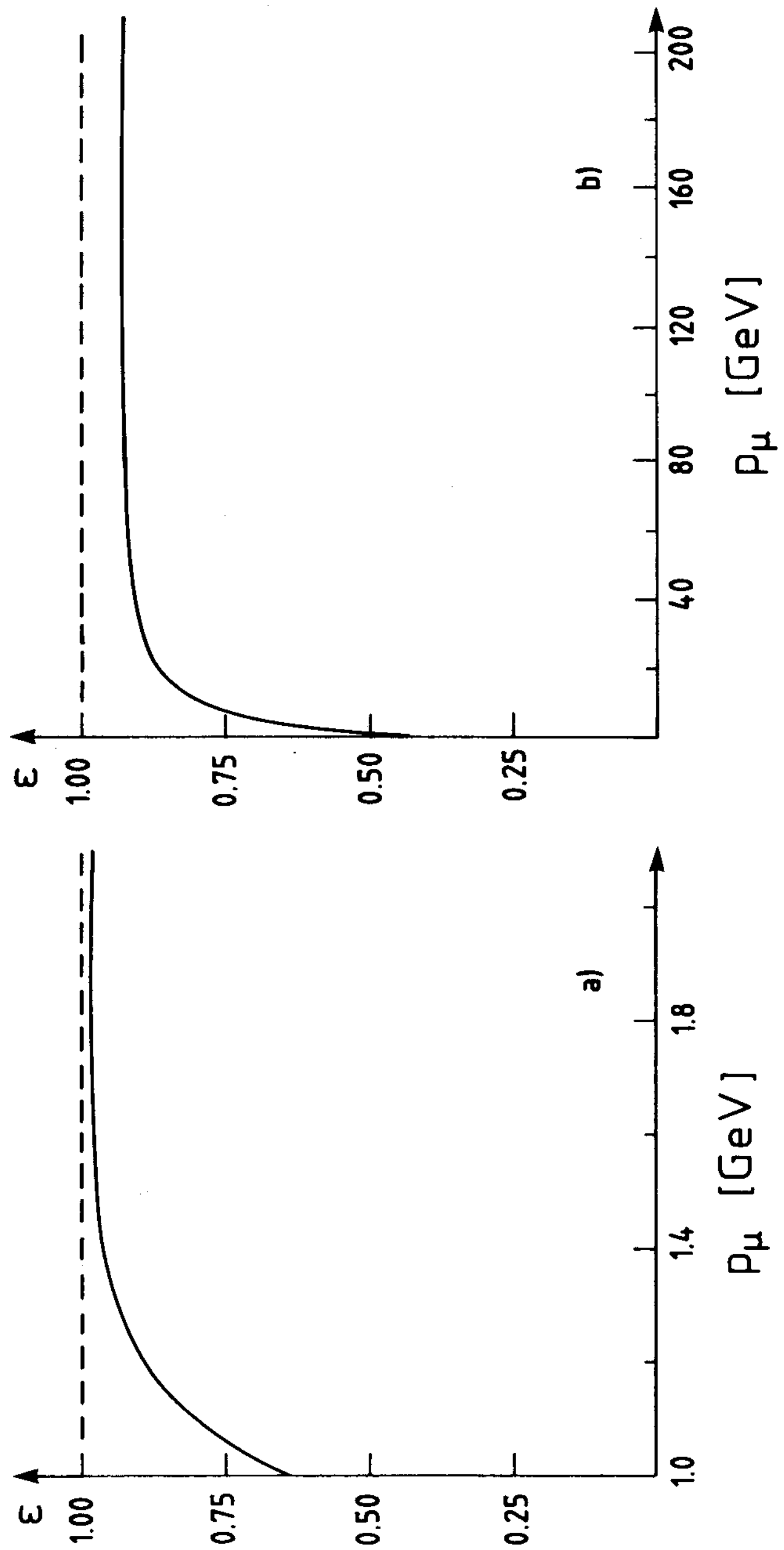

$\stackrel{0}{0}$ 


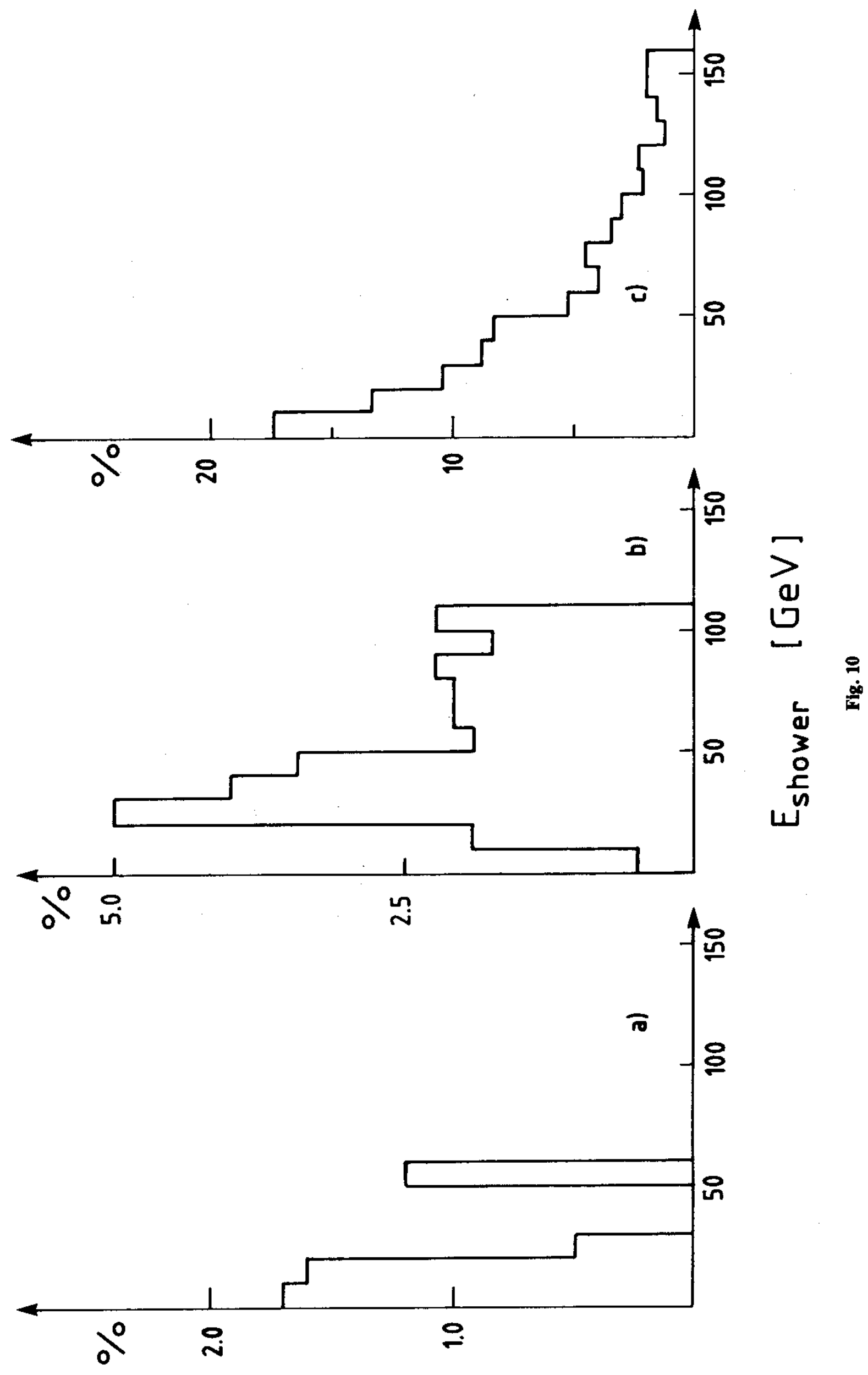




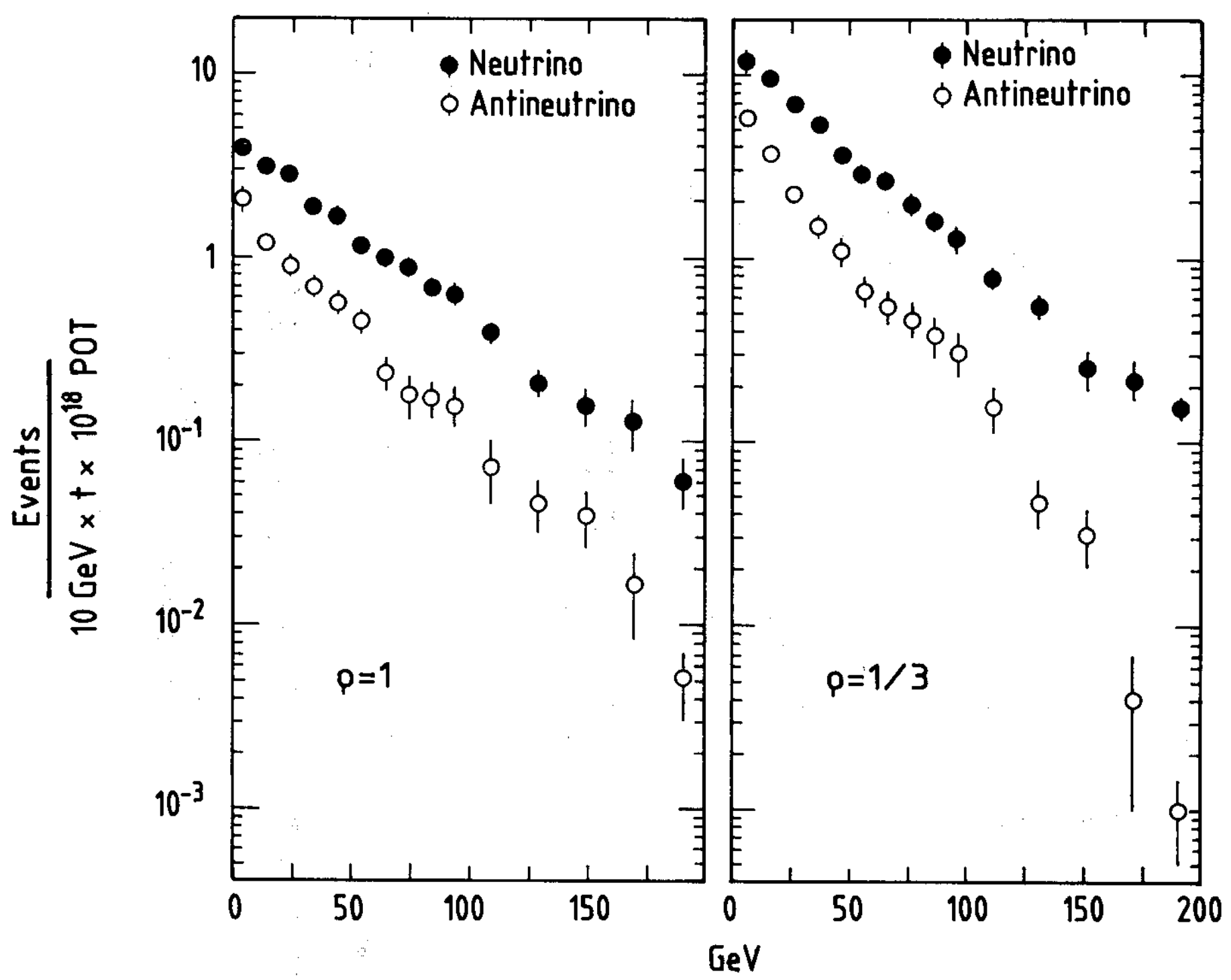

Fig. 11 


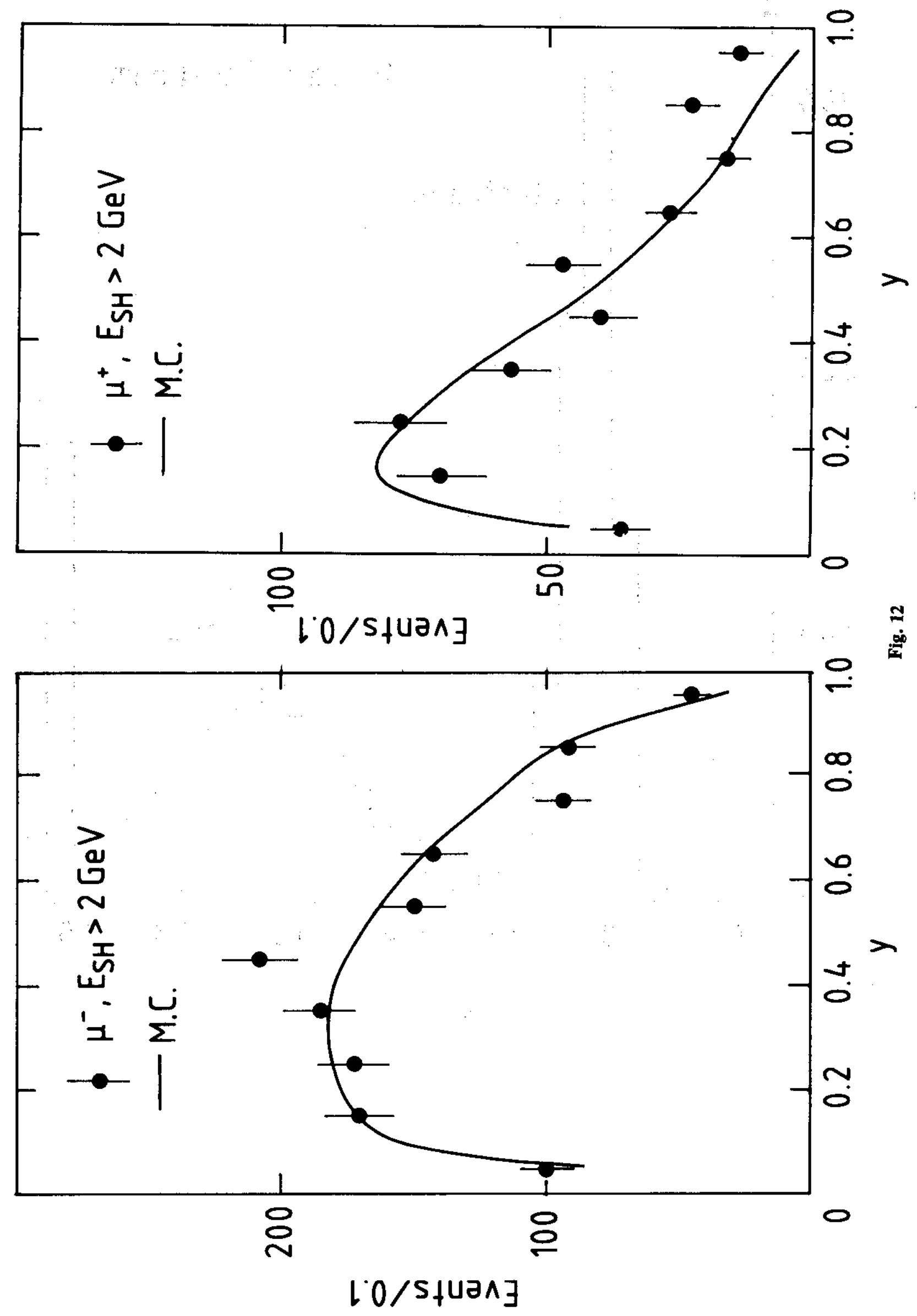




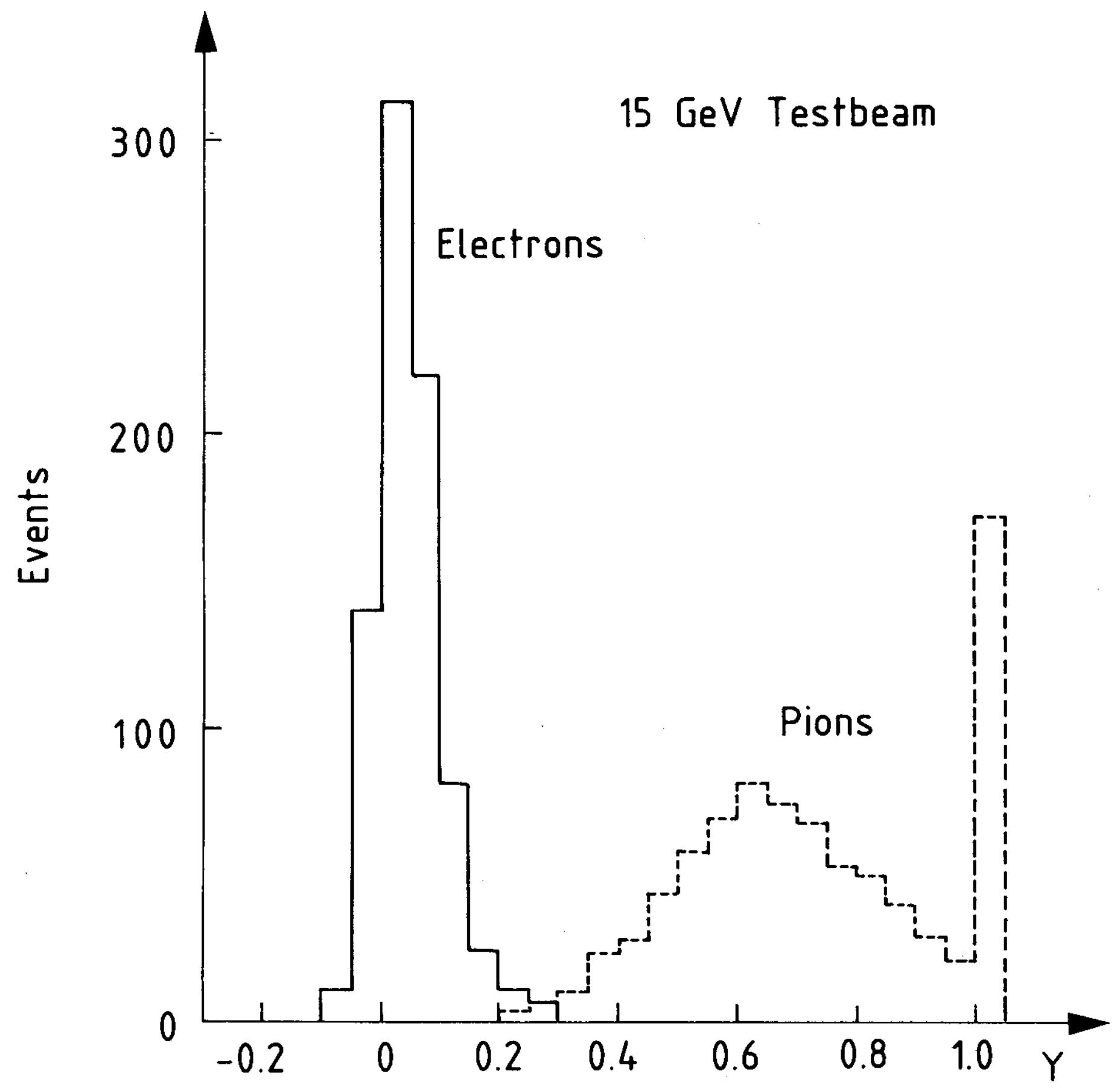

Fig. 13 


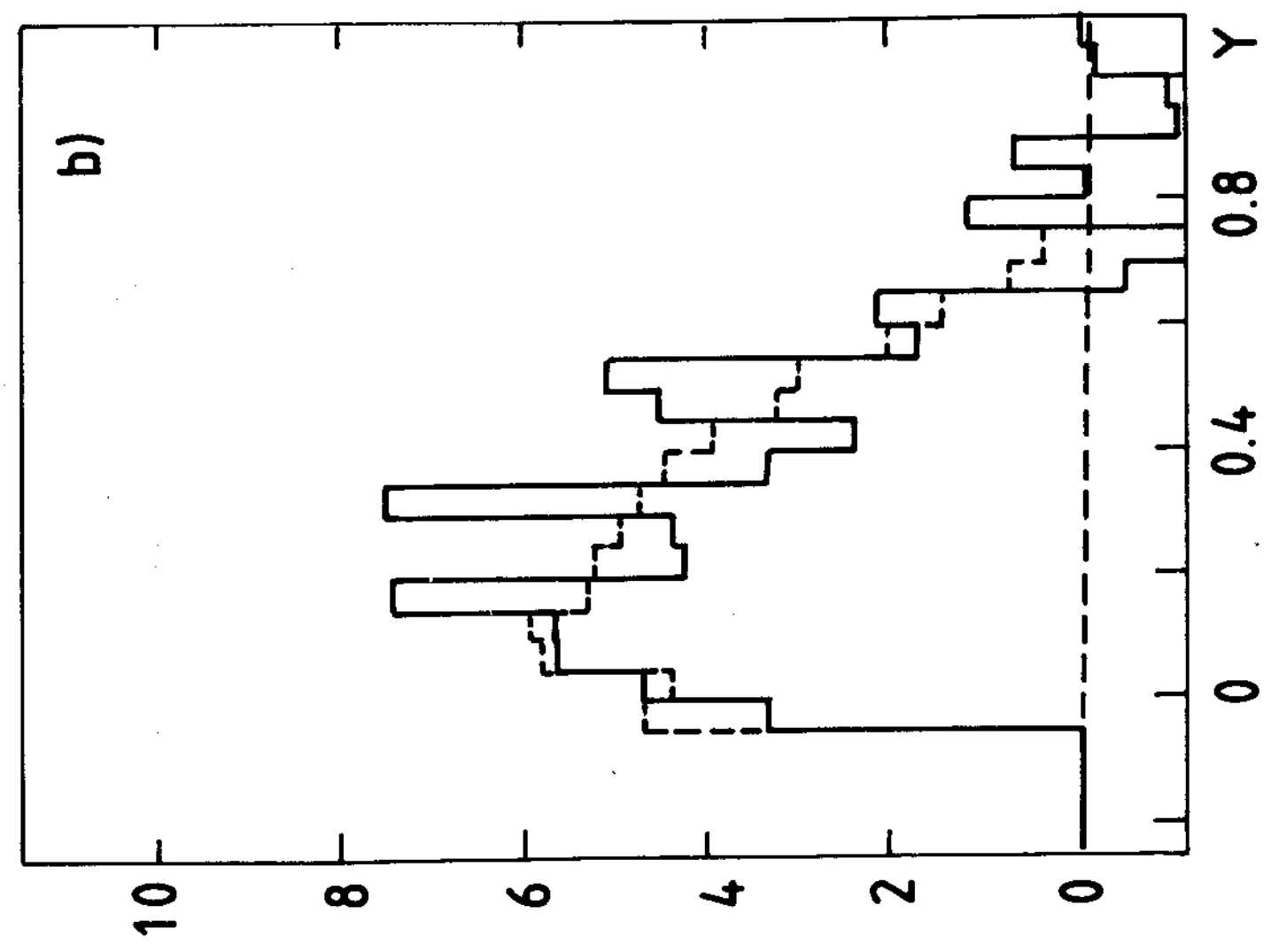

$\stackrel{\square}{ \pm}$

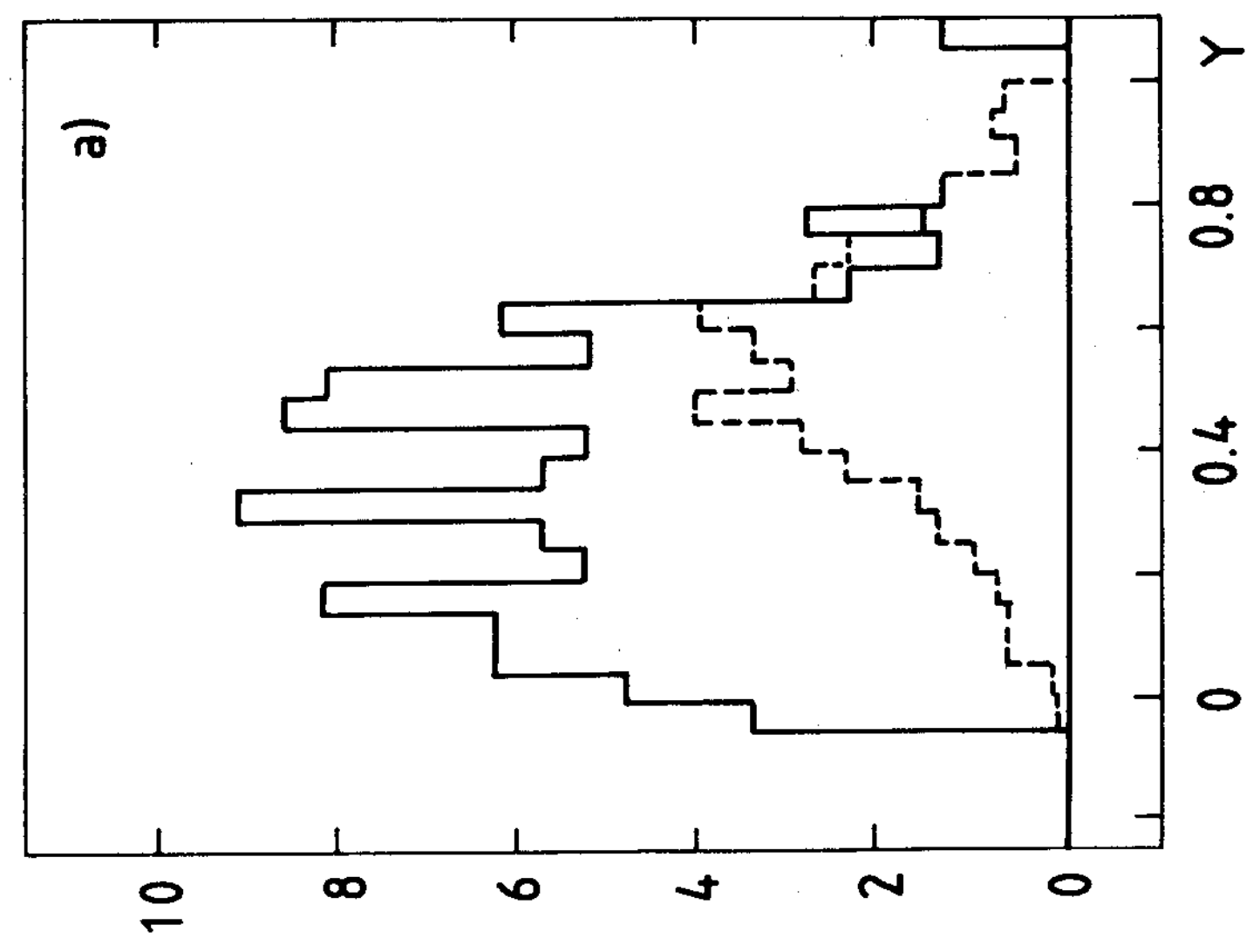

(st!un Kjejt!que) squanz 


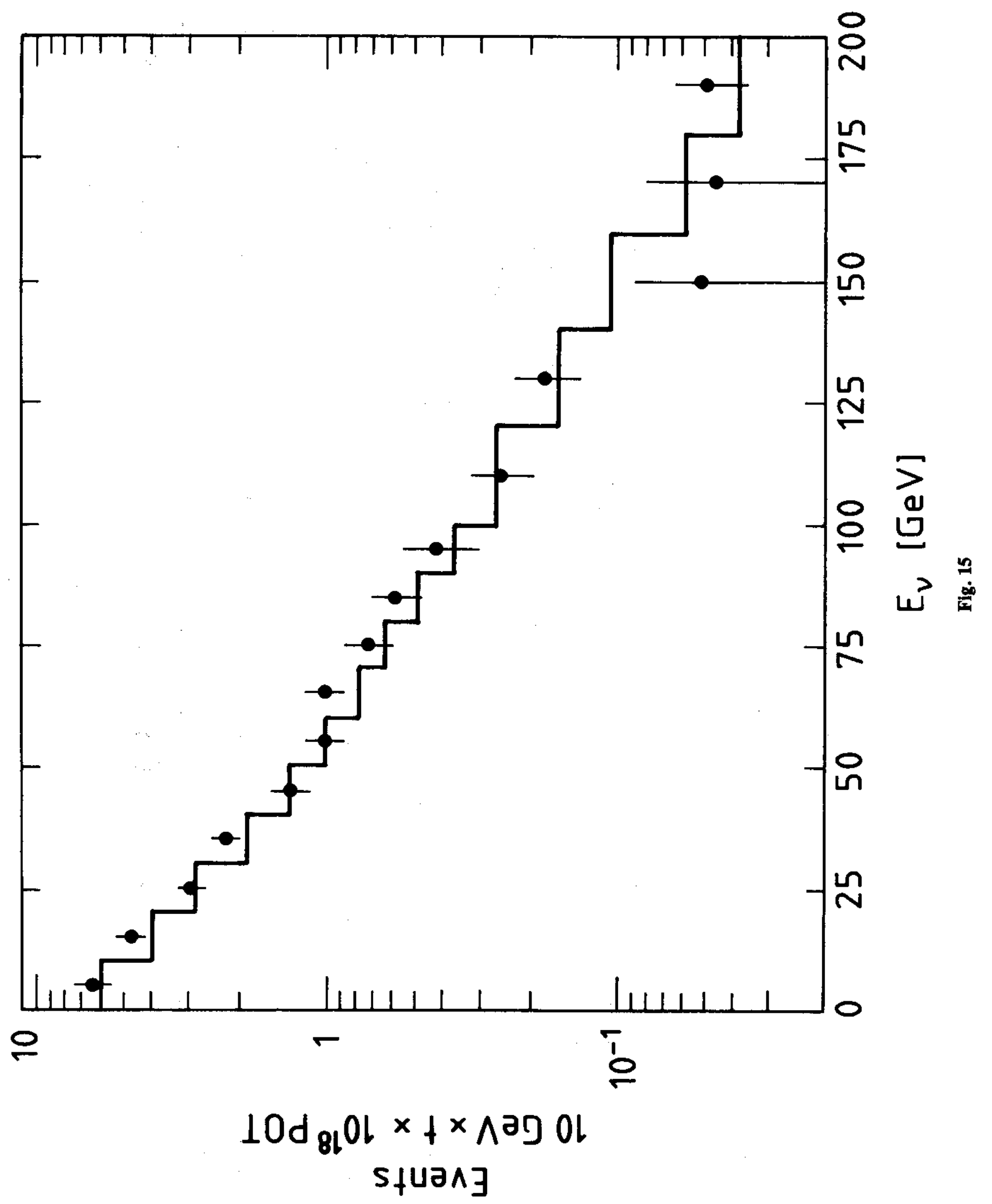




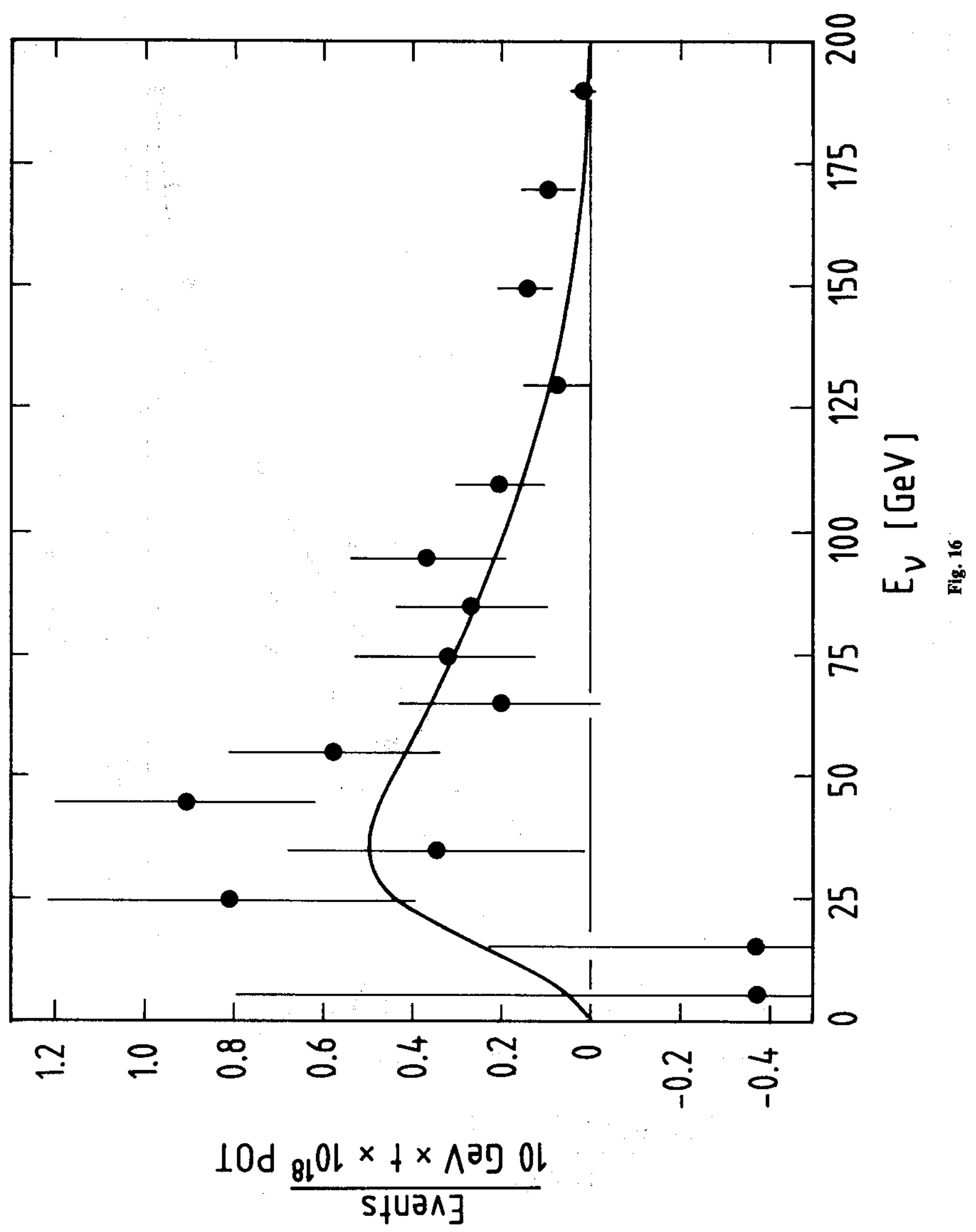




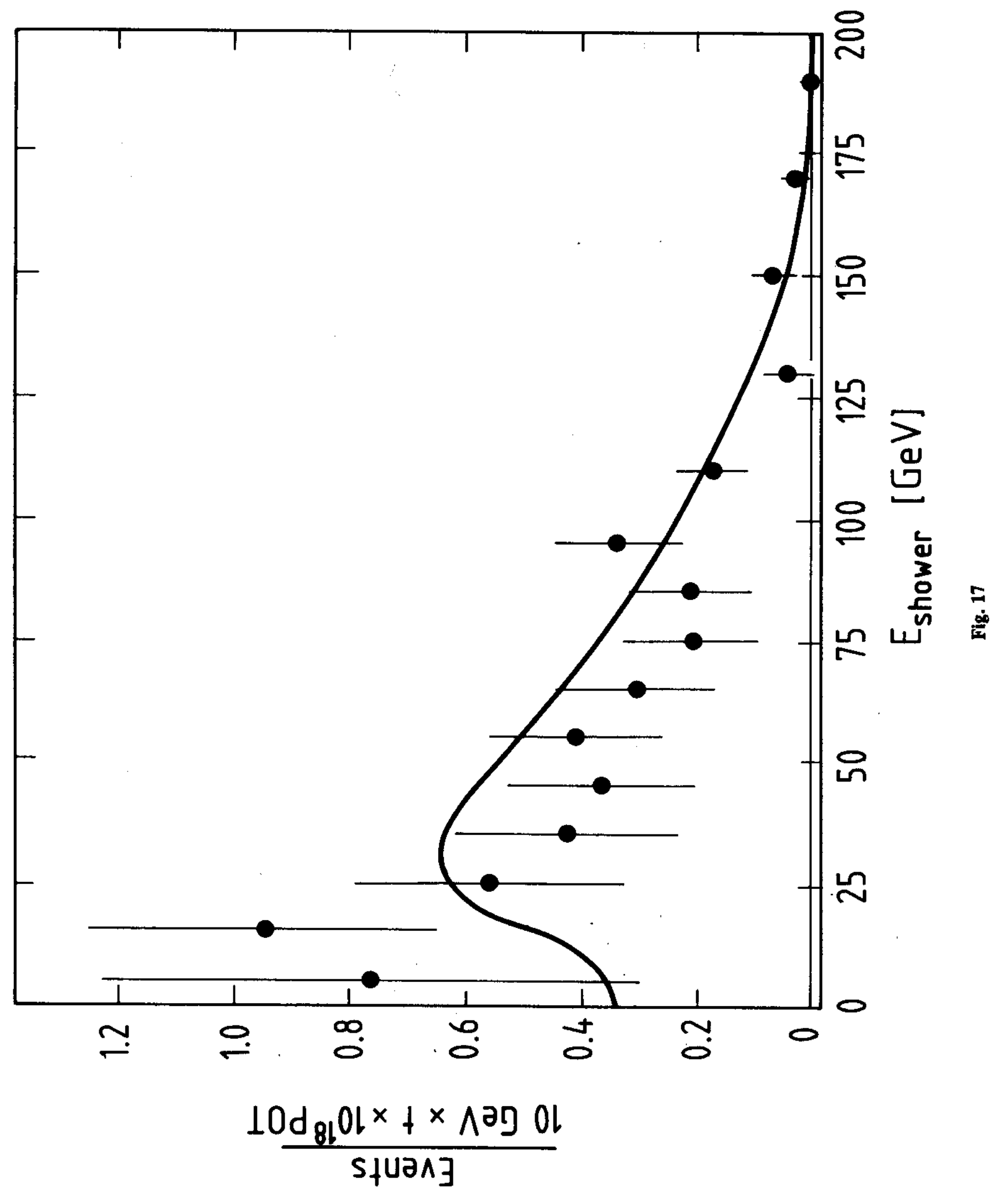




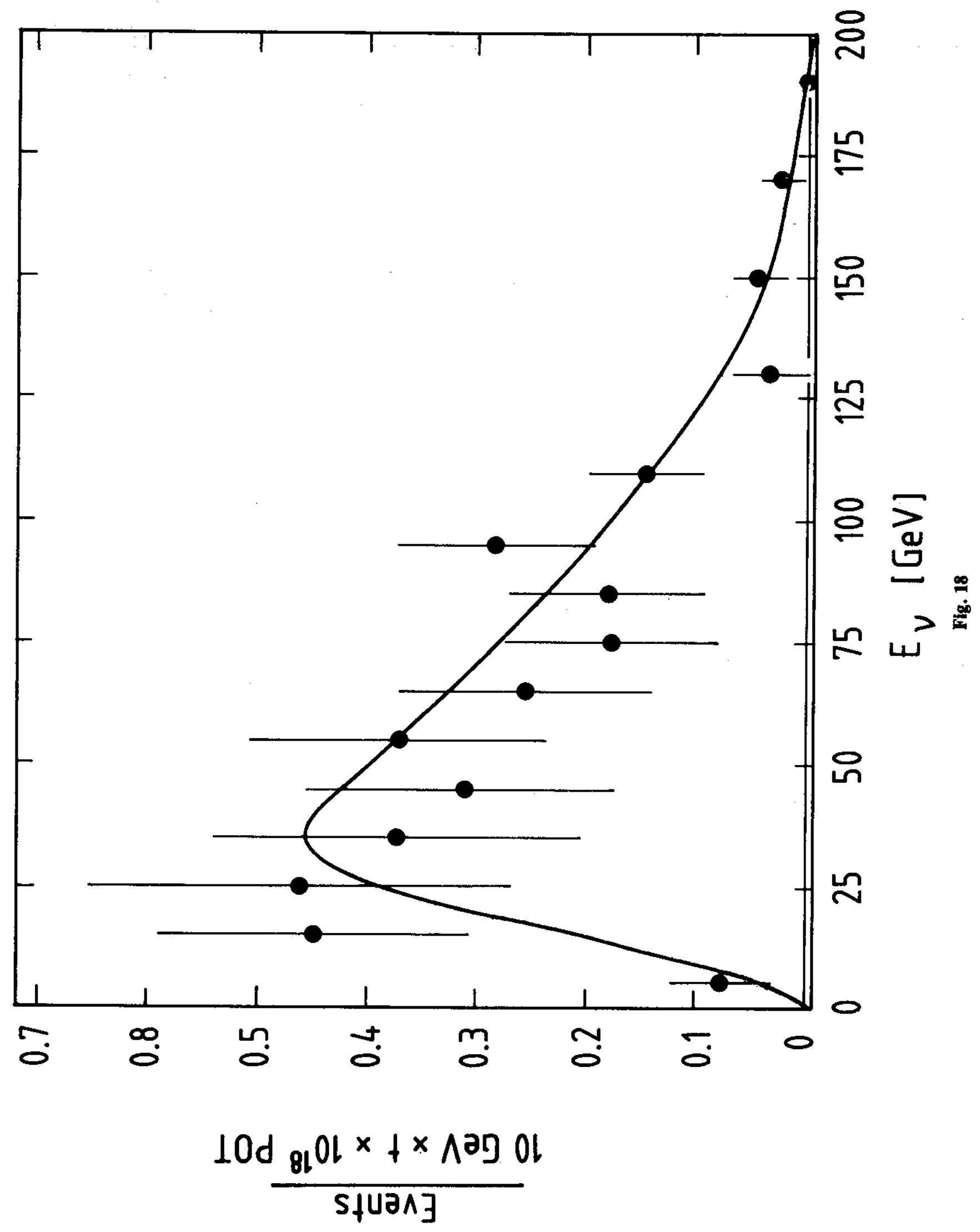




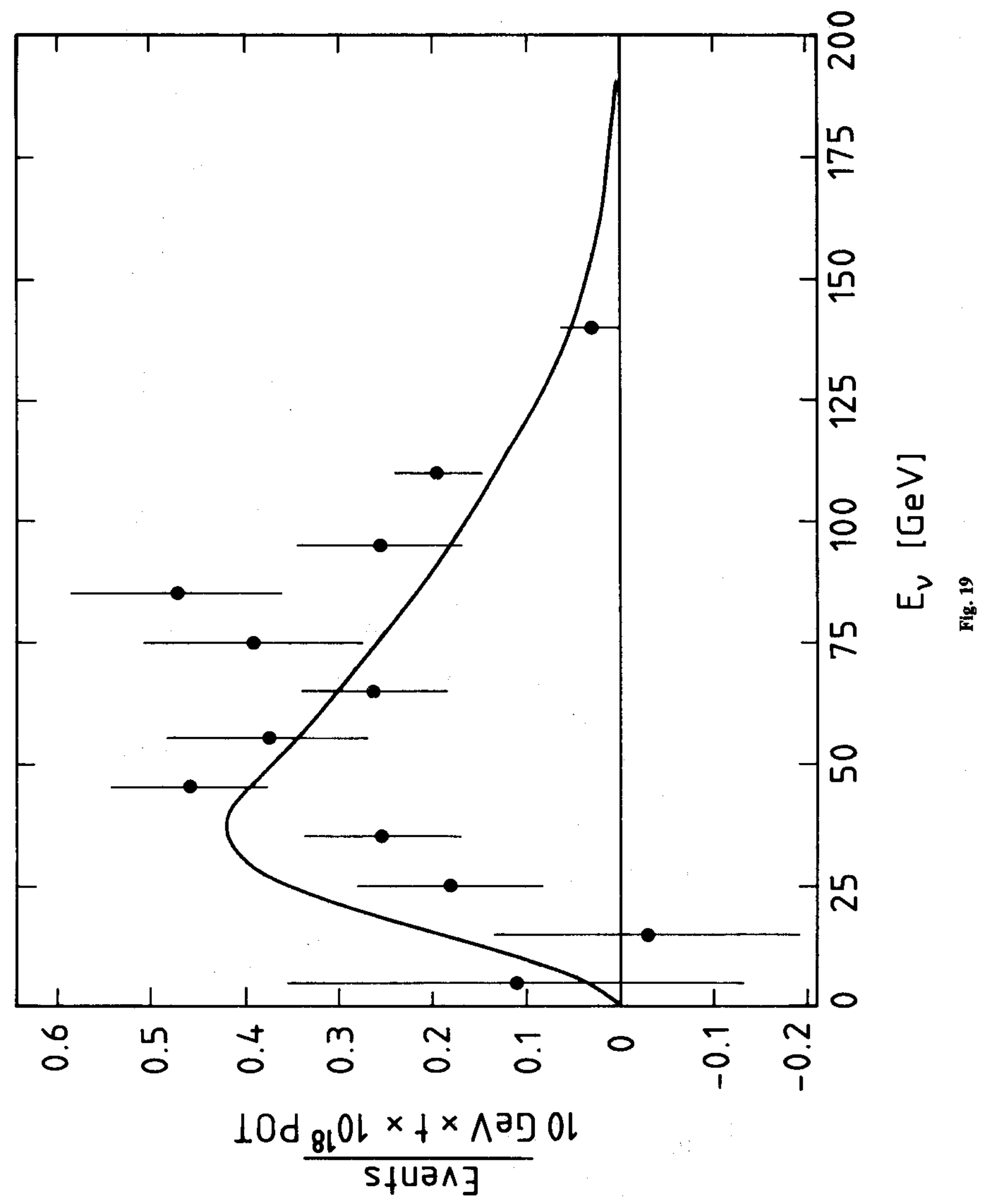




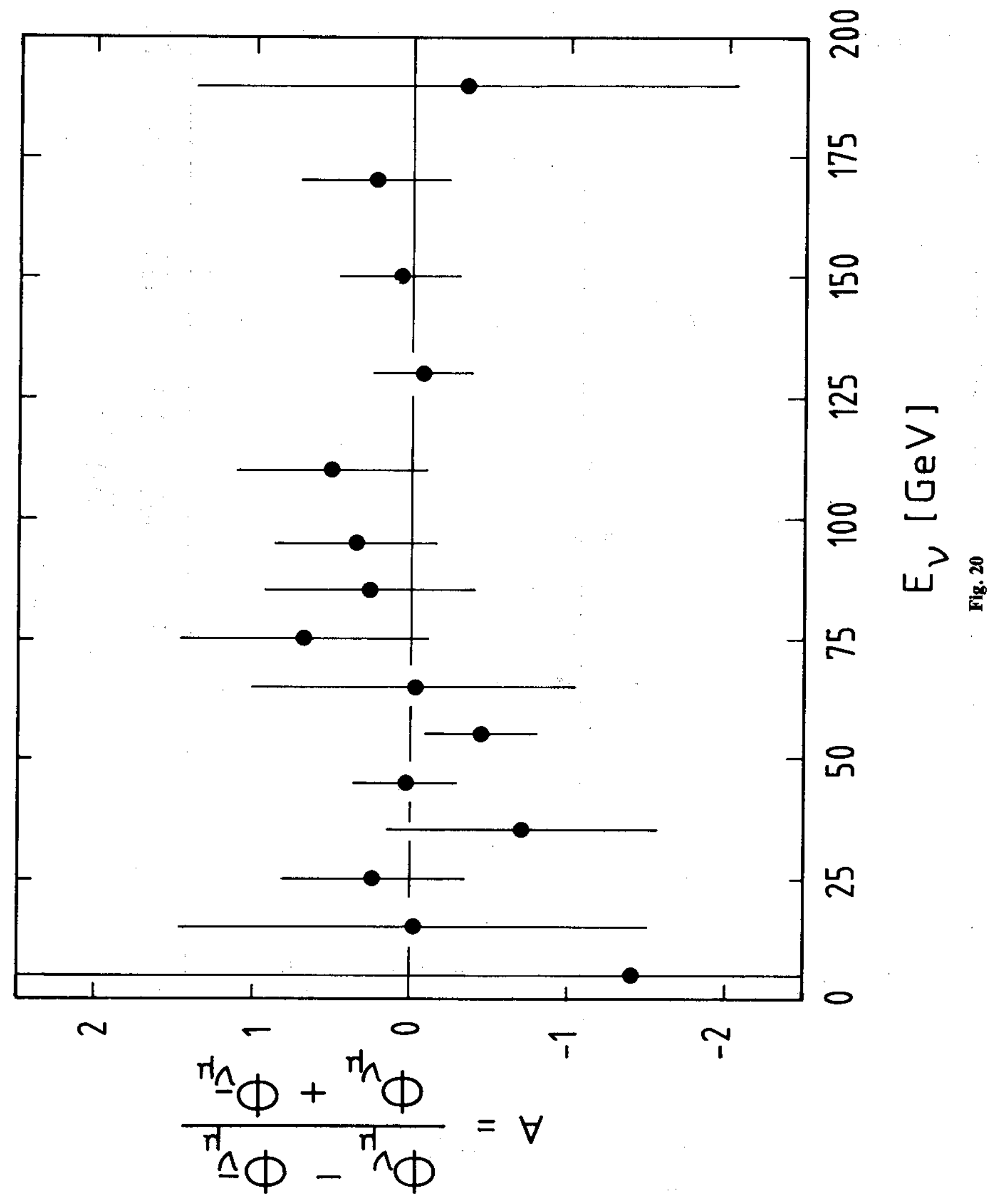




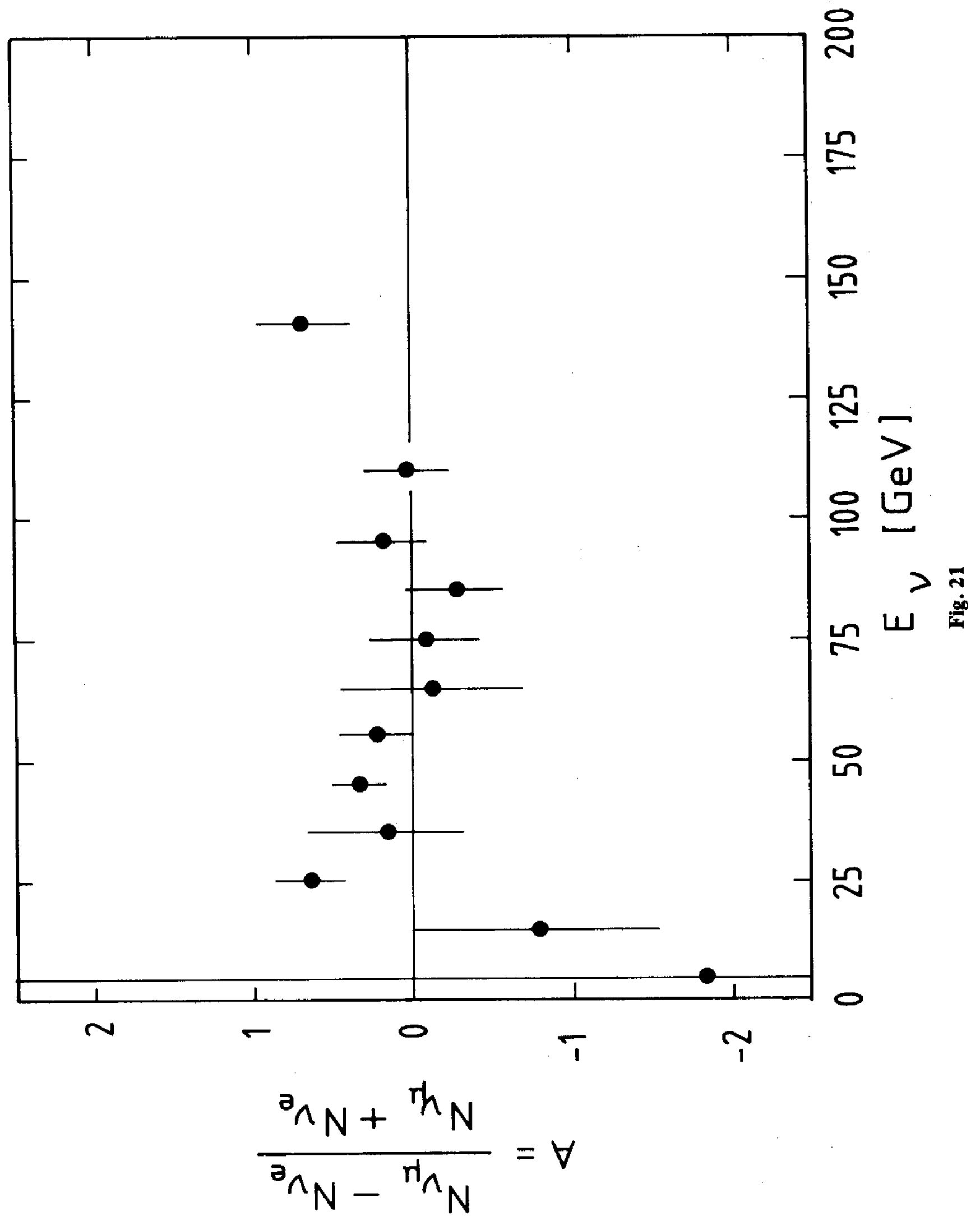

